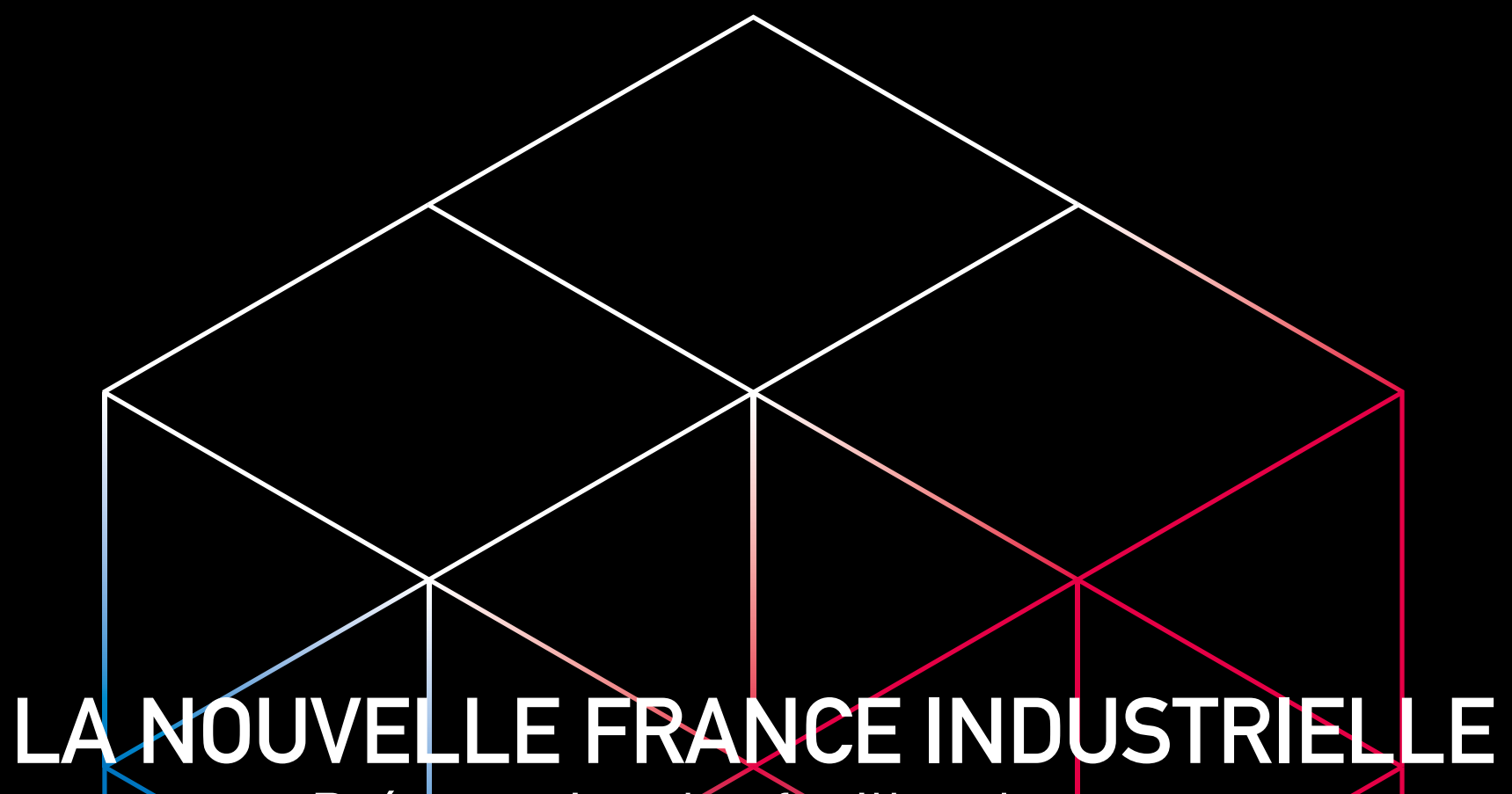

Présentation des feuilles de route des 34 plans de ta nouvelle France industrielle
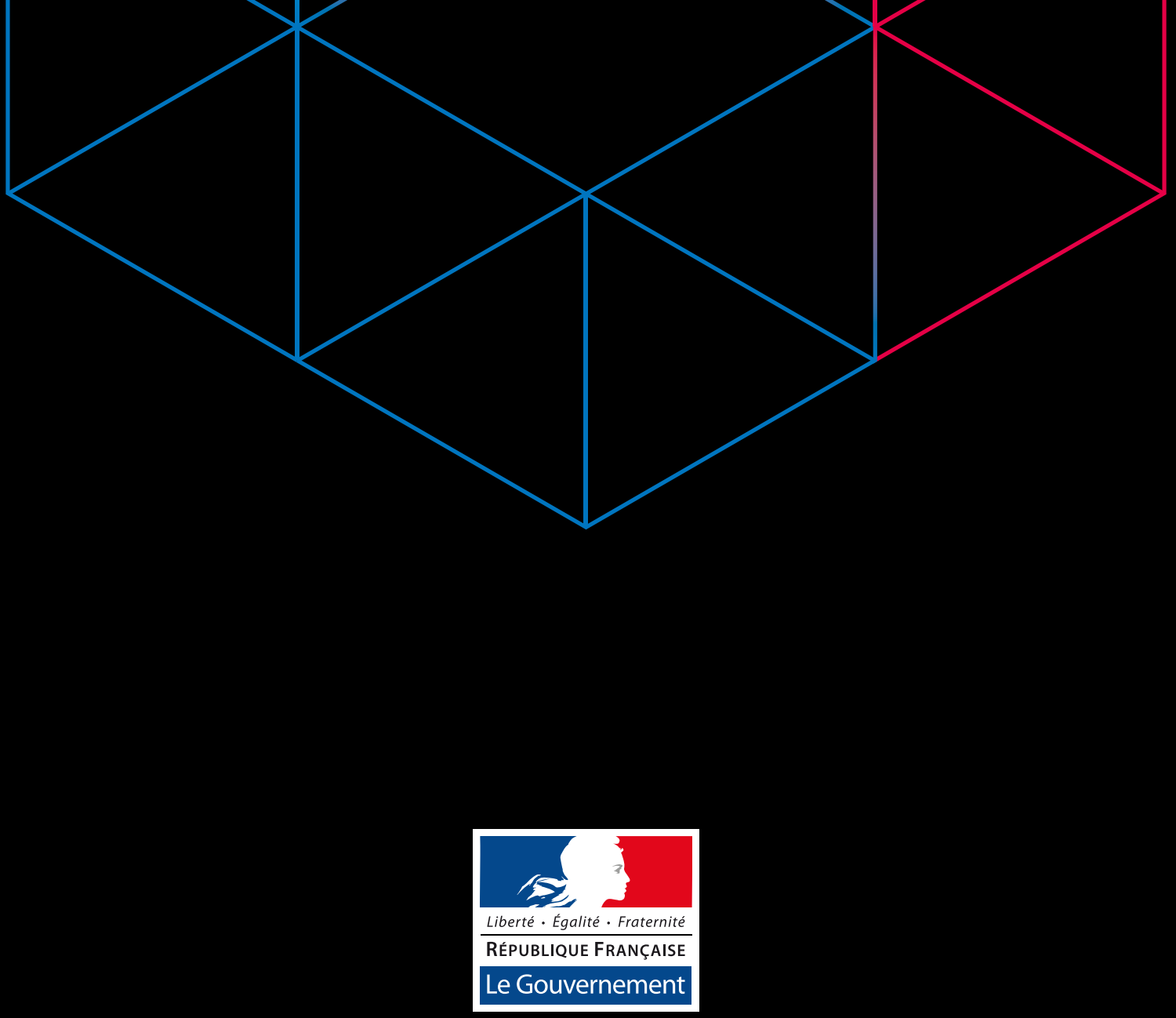


\section{PLANS DE RECONQUÊTE POUR LA NOUVELLE FRANCE INDUSTRIELLE}

Le 12 septembre 2013, le Président de la République et le Ministre de l'Economie, de l'Industrie et du Numérique lançaient conjointement à l'Élysée 34 plans de reconquête industrielle, les 34 plans de la nouvelle France industrielle. Ils remettaient dans les cœurs et dans les têtes l'idée que la France est une grande nation industrielle, solide, productive, capable de se réinventer et de devenir pionnière dans la troisième révolution industrielle, au carrefour des transitions écologique et énergétique d'une part, digitale et numérique d'autre part. L'ambition était de rassembler les acteurs économiques et industriels autour d'un objectif commun, de mettre les outils de l'État au service de ce dessein et de mobiliser les écosystèmes locaux autour de la construction d'une offre industrielle française nouvelle et compétitive, capable de gagner des parts de marché en France et à l'international et de créer ainsi des emplois nouveaux. — C'est aujourd'hui chose faite. Neuf mois après leur lancement, tous les plans sont en ordre de marche. Leurs feuilles de route, présentées dans ce recueil, ont toutes été validées avant le 9 juillet 2014, par un comité de pilotage réunissant des acteurs publics et privés, sous l'autorité du Premier Ministre. Celles-ci déclinent dans un calendrier précis des actions concrètes, publiques et privées, et détaillent le rôle respectif des différents acteurs et les objectifs à atteindre : de nouvelles offres, de nouvelles ruptures technologiques, de nouveaux investissements et de nouveaux emplois. - Ce n'est qu'une étape mais déjà, les travaux accomplis grâce à cette alliance inédite entre les pouvoirs publics et les entreprises sont exceptionnels et particulièrement innovants. Plus de 500 personnes se sont investies dans les équipes projets, représentant plus de 250 entreprises, majoritairement des PME et des ETI. Véritables chefs d'orchestre, les chefs de projet ont été les organisateurs du travail collectif et des projets collaboratifs. Tous se sont montrés à la hauteur des enjeux et exercent leur tâche avec responsabilité. Qu'ils en soient ici remerciés. - Ces 34 leviers de croissance pour notre pays entrent désormais dans leur phase la plus cruciale, celle de leur mise en œuvre opérationnelle. Le Gouvernement la suivra avec détermination et constance et compte sur la mobilisation de chacun aux côtés des industriels pour atteindre les objectifs ambitieux qui ont été fixés, pour la France et pour la reconquête industrielle. 


\section{SOMMAIRE}

ÉNERGIES RENOUVELABLES

LA VOITURE POUR TOUS CONSOMMANT MOINS DE 2 LITRES AUX $100 \mathrm{KM}$ BORNES ÉLECTRIQUES DE RECHARGE AUTONOMIE ET PUISSANCE DES BATTERIES VÉHICULE AUTONOME AVION ÉLECTRIQUE ET NOUVELLE GÉNÉRATION D'AÉRONEFS DIRIGEABLES ET DRONES CIVILS LOGICIELS ET SYSTĖMES EMBARQUÉS LE SATELLITE À PROPULSION ÉLECTRIQUE TGV DU FUTUR NAVIRES ÉCOLOGIQUES TEXTILES TECHNIQUES ET INTELLIGENTS INDUSTRIES DU BOIS RECYCLAGE ET MATÉRIAUX VERTS RÉNOVATION THERMIQUE DES BÂTIMENTS RÉSEAUX ÉLECTRIQUES INTELLIGENTS QUALITÉ DE L'EAU ET GESTION DE LA RARETÉ CHIMIE VERTE ET BIOCARBURANTS
BIOTECHNOLOGIES MÉDICALES

45 SANTÉ NUMÉRIQUE

47 DISPOSITIFS MÉDICAUX ET NOUVEAUX ÉQUIPEMENTS DE SANTÉ

49 PRODUITS INNOVANTS POUR UNE ALIMENTATION SÛRE, SAINE ET DURABLE

51 BIG DATA

53 CLOUD COMPUTING

55 E-ÉDUCATION

57 SOUVERAINETÉ TÉLÉCOMS

59 NANOÉLECTRONIQUE

61 OBJETS CONNECTÉS

63 RÉALITÉ AUGMENTÉE

65 SERVICES SANS CONTACT

67 SUPERCALCULATEURS

69 ROBOTIQUE

71 CYBERSÉCURITÉ

73 USINE DU FUTUR

75 LES CHEFS DE PROJET

76 GLOSSAIRE 


\section{ÉQUIPE DU PLAN}

CHEF DE PROJET

Jean-Claude Andréini est vice-président du Comité stratégique des éco-industries (COSEI).

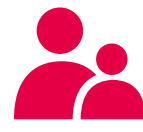

LES INDUSTRIELS

Alstom Renewable Power, Areva, DCNS, EDF EN, GDF-Suez, Schneider Electric,

Total ENR, CNIM, CIAT, Poujoulat, Quadran, ECM Technologies, Urbasolar

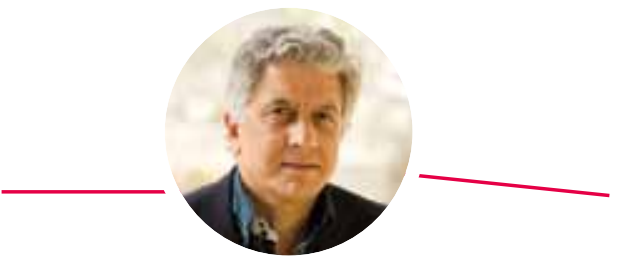

LES ACTEURS PUBLICS MEIN, MEDDE, DGCIS, DGEC, Bpifrance, CGI, ADEME, Interpôle des pôles de compétitivité énergie, CEA-LITEN

\section{SYNTHÈSE DES ACTIONS DU PLAN}

\begin{tabular}{|c|c|c|}
\hline Action & Calendrier & Engagement/objectif \\
\hline $\begin{array}{l}\text { Réalisation d'une cartographie stratégique } \\
\text { des forces françaises }\end{array}$ & $\begin{array}{l}\text { S2 } 2014 \text { (définition), } \\
\text { Mi } 2015 \text { (livrable) }\end{array}$ & $\begin{array}{l}\text { Mise à disposition des données des industriels pour constituer un } \\
\text { support cartographié dynamique. Etude cofinancée Etat-industriels ; } \\
\text { budget estimé de } 90 \text { à } 200 \mathrm{~K} €\end{array}$ \\
\hline $\begin{array}{l}\text { Établissement d'une maitrise d'œuvre } \\
\text { pour un suivi partagé des projets/actions }\end{array}$ & Continu & $\begin{array}{l}\text { Constitution d'une équipe opérationnelle en appui de l'équipe projet } \\
\text { du plan (syndicat des énergies renouvelables) }\end{array}$ \\
\hline \multicolumn{3}{|l|}{ Ambition Export } \\
\hline $\begin{array}{l}\text { Définition d'un plan d'action export : } \\
\text { vitrines technologiques françaises et } \\
\text { comités export par pays }\end{array}$ & $\begin{array}{l}\text { Janvier } 2015 \\
\text { (mise en place en) }\end{array}$ & $\begin{array}{l}\text { Désignation de cinq pays cibles prioritaires, constitution d'équipes } \\
\text { dédiées sous coordination du PEXE, l'association des éco-entreprises } \\
\text { de France, valoriser les DOM et les territoires ultramarins (Sea-Water } \\
\text { Air Conditioning, etc.) } \\
\text { Coûts estimés pour cinq pays: } 1 \mathrm{M€} \\
\text { Financement: industriels et dispositifs BPI, AFII-UBI, etc... }\end{array}$ \\
\hline \multicolumn{3}{|c|}{ Ambition « structuration des filières » et « Mittelstand » des ENR } \\
\hline \multirow[t]{2}{*}{$\begin{array}{l}\text { Renforcement du développement d'un } \\
\text { écosystème d'ETI et de PME performantes }\end{array}$} & Dès 2014 & $\begin{array}{l}\text { Accompagnement des projets de structuration de filières } \\
\text { (par exemple: PAC et Solaire Thermique ainsi que Biogaz } \\
\text { Méthanisation, en vue de mener de front l'augmentation de l'activité } \\
\text { et la part de valeur ajoutée créatrice d'emploi en France) }\end{array}$ \\
\hline & & $\begin{array}{l}\text { Dispositifs de soutien publics à l'innovation et aux filières grâce } \\
\text { aux outils Bpifrance (aides à l'innovation, financement, garantie, } \\
\text { investissement en fonds propres, accompagnement à l'export) } \\
\text { et autres banques et acteurs financiers }\end{array}$ \\
\hline $\begin{array}{l}\text { Création d'un fonds assurantiel } \\
\text { pour la géothermie }\end{array}$ & 2015 & $\begin{array}{l}\text { Mutualisation du risque géotechnique amont des projets pour } \\
\text { optimiser l'offre collective de « l'équipe de France ". } \\
\text { Objectif } 20 \text { projets et } 1000 \text { emplois directs induits }\end{array}$ \\
\hline
\end{tabular}




\section{ÉNERGIES RENOUVELABLES}
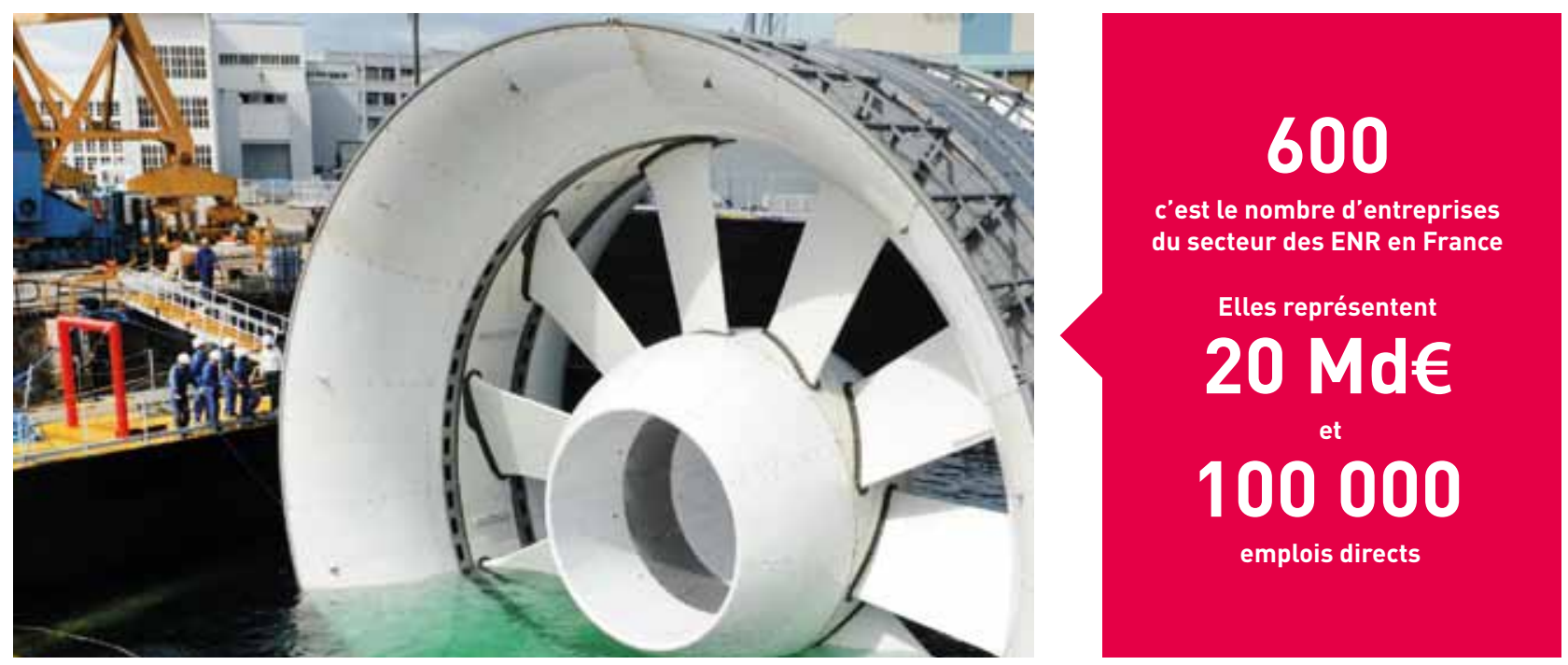

Hydrolienne DCNS

- Nous construisons la France des énergies renouvelables, moins dépendante aux hydrocarbures. La transition énergétique implique de renforcer les filières industrielles nationales matures. Il s'agit également de porter les autres à la compétitivité économique, de réduire les importations d'énergies fossiles et d'inverser le solde commercial des équipements pour l'énergie, gage de création de valeur ajoutée et d'emplois sur le territoire. Le plan doit relever trois défis: diminuer les prix de revient des énergies les plus matures (éolien terrestre ou photovoltaïque), asseoir notre leadership technologique sur les énergies de rupture comme les énergies marines, la géothermie ou le solaire photovoltaïque à concentration, déployer les énergies thermiques autour de produits innovants à coût abordable mis au point par nos industriels. - Pour ce faire, le plan s'assigne trois ambitions : dessiner la cartographie dynamique des industries des ENR, accélérer leur développement à l'international et promouvoir un écosystème de PME et ETI performantes. Il concourra ainsi à l'objectif majeur du contrat de filière des énergies renouvelables : créer 125000 emplois supplémentaires d'ici 2020. - L'État apporte ses leviers de politique industrielle, notamment des investissements d'avenir, et œuvre à une évolution maitrisée de l'environnement réglementaire pour faire de la France un authentique territoire d'accueil des nouvelles énergies. - Le plan s'inscrit dans une dynamique de projets structurants pour l'innovation et le renforcement en fonds propres des entreprises qu'il prolonge et amplifie. Au démarrage de la démarche, il a suscité plus de vingt nouveaux projets. Il se dote d'une gouvernance de suivi des initiatives et de la structuration du secteur pour agir dans la durée. 


\section{ÉQUIPE DU PLAN}

CHEFS DE PROJET

Jean-Michel Billig est directeur des Ingénieries et de la Qualité du groupe Renault.

Gilles Le Borgne est directeur Recherche et Développement du groupe PSA Peugeot Citroën.

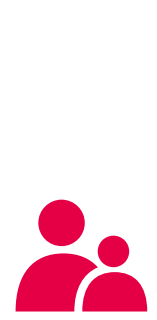

LES ÉCOLES ET ORGANISMES DE RECHERCHE CEA, IFP EN

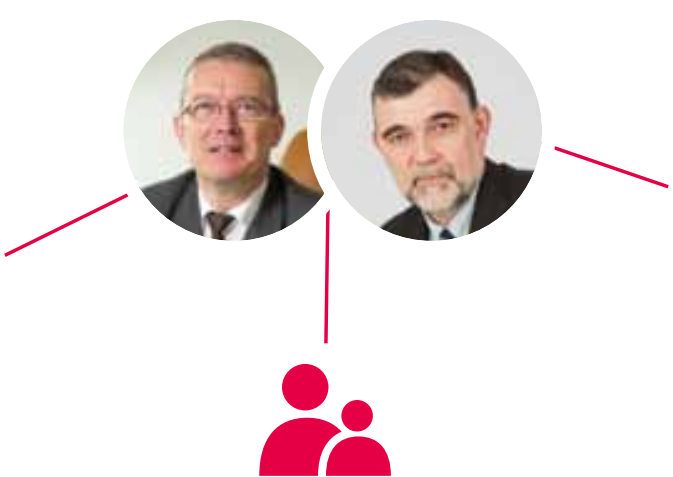

LES ACTEURS PUBLICS

Bpifrance, CGI, MENESR, MEDDE, MEIN

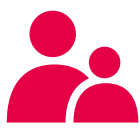

LES INDUSTRIELS

Renault, PSA, Faurecia, Michelin, Plastic Omnium, Valeo, PME et ETI de la filière automobile, Plateforme Automobile (PFA)

\section{SYNTHĖSE DES ACTIONS DU PLAN}

\begin{tabular}{|c|c|c|c|c|}
\hline Action & Pilote & Calendrier & Acteurs privés & Acteurs publics et leviers d'action \\
\hline $\begin{array}{l}\text { Démonstration de } \\
\text { la faisabilité d'un } \\
\text { véhicule } 21 / 100 \mathrm{~km} \\
\text { à coût abordable }\end{array}$ & $\begin{array}{l}\text { Plateforme } \\
\text { automobile } \\
\text { (PFA) }\end{array}$ & Achevée en 2013 & $\begin{array}{l}\text { PSA et Renault pour } \\
\text { la synthèse } \\
\text { Faurecia, Valeo, Michelin, } \\
\text { Plastic Omnium pour les } \\
\text { briques technologiques }\end{array}$ & $\begin{array}{l}\text { CGI, Ministères et Ademe } \\
\text { Investissements d'Avenir } \\
\text { (programme Véhicule routier } \\
\text { du futur doté de } 250 \mathrm{M} € \text { ) }\end{array}$ \\
\hline $\begin{array}{l}\text { Réalisation de projets } \\
\text { de R\&D rassemblant } \\
\text { l'ensemble de la } \\
\text { filière automobile sur } \\
\text { les quatre briques } \\
\text { technologiques } \\
\text { identifiées }\end{array}$ & $\begin{array}{l}\text { Plateforme } \\
\text { automobile } \\
\text { (PFA) }\end{array}$ & $\begin{array}{l}\text { Appel à } \\
\text { manifestations } \\
\text { d'intérêt «Véhicule } \\
\text { routier du futur» } \\
2014\end{array}$ & $\begin{array}{l}\text { Ensemble de la filière } \\
\text { automobile: } \\
\text { grands groupes industriels } \\
\text { français et étrangers, des } \\
\text { PME/ETI ayant des centres } \\
\text { de R\&D en France } \\
\text { CEA, IFPEN, laboratoires } \\
\text { publics }\end{array}$ & $\begin{array}{l}\text { CGI, Ministères, Ademe } \\
\text { Investissements d'Avenir } \\
\text { (programme Véhicule routier } \\
\text { du futur doté de } 250 \mathrm{M} € \text { ) }\end{array}$ \\
\hline $\begin{array}{l}\text { Intégration des } \\
\text { différents systèmes } \\
\text { et validation de } \\
\text { l'atteinte du cahier } \\
\text { des charges sur des } \\
\text { démonstrateurs de } \\
\text { synthèse }\end{array}$ & PSA et Renault & $\begin{array}{l}\text { Présentation } \\
\text { des premiers } \\
\text { démonstrateurs lors } \\
\text { du Mondial de Paris } \\
\text { en } 2014\end{array}$ & $\begin{array}{l}\text { PSA et Renault avec } \\
\text { les contributions } \\
\text { des équipementiers, } \\
\text { ETI et PME de la filière }\end{array}$ & \\
\hline
\end{tabular}




\section{LA VOITURE POUR TOUS CONSOMMANT MOINS DE 2 LITRES AUX 100 KM}
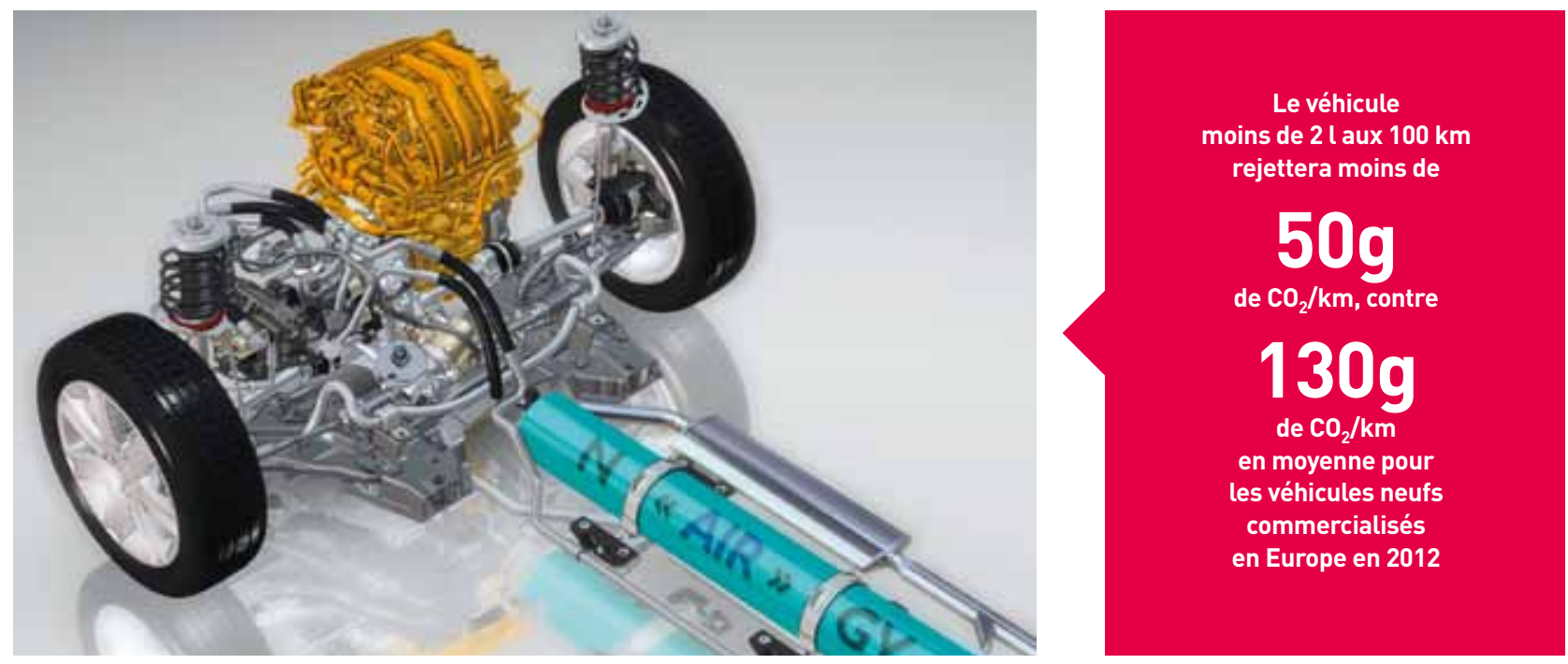

Chaîne de traction HYbrid Air, PSA

Nous construisons la France des véhicules écologiques, économiques et populaires, consommant moins de 2 I aux $100 \mathrm{~km}$. L'objectif de commercialiser des véhicules de série consommant moins de 2 l aux $100 \mathrm{~km}$ mais avec un prix accessible au plus grand nombre est un vrai défi. Il s'agit de parvenir à une émission moyenne d'environ $50 \mathrm{~g}$ de $\mathrm{CO}_{2}$ par kilomètre, alors qu'en 2012 la moyenne des émissions de $\mathrm{CO}_{2}$ des véhicules neufs commercialisés en Europe dépassait $130 \mathrm{~g}$ par kilomètre. Les véhicules visés prioritairement sont ceux du «segment B » (type CLIO, 208, C3), qui représente environ 30 \% des ventes de véhicules particuliers neufs en Europe. Toutefois, les innovations développées dans le cadre du plan permettant de réduire la consommation et les émissions de $\mathrm{CO}_{2}$ seront également utilisables sur l'ensemble des gammes. - Le plan $2 \mathrm{l} / 100 \mathrm{~km}$ passe dans un premier temps par le développement de briques technologiques, permettant de réaliser des ruptures en termes de réduction de $\mathrm{CO}_{2}$ à coût acceptable pour le client. Les premières briques seront industrialisées dès 2017, ce qui rendra possible la commercialisation en grande série des premiers véhicules 2 l/100 km avant 2020 .

- Le plan fédère l'ensemble de la filière automobile et au-delà. Grands groupes, PME, ETI, laboratoires publics, plus de 160 entités ont déjà fait part de leur intérêt pour rejoindre le programme sur ces thématiques de recherche. Le montant total des investissements R\&D à engager est, à ce stade, évalué à environ un milliard d'euros sur les 5 ans à venir. L'État accompagne ces efforts, au travers notamment du programme des investissements d'avenir qui a lancé un nouvel appel à projets doté de 250 millions d'euros d'aides. Une vingtaine d'autres projets, représentant au total plus de 350 millions d'euros de dépenses de R\&D, feront l'objet d'une décision cette année. - Dès le Mondial de l’Automobile 2014, une première étape concrète sera franchie par la présentation par les constructeurs français de véhicules prototypes servant de support aux technologies nécessaires pour atteindre la cible des 2 l/100 km. 


\section{ÉQUIPE DU PLAN}

CHEF DE PROJET

Francis Vuibert est préfet.

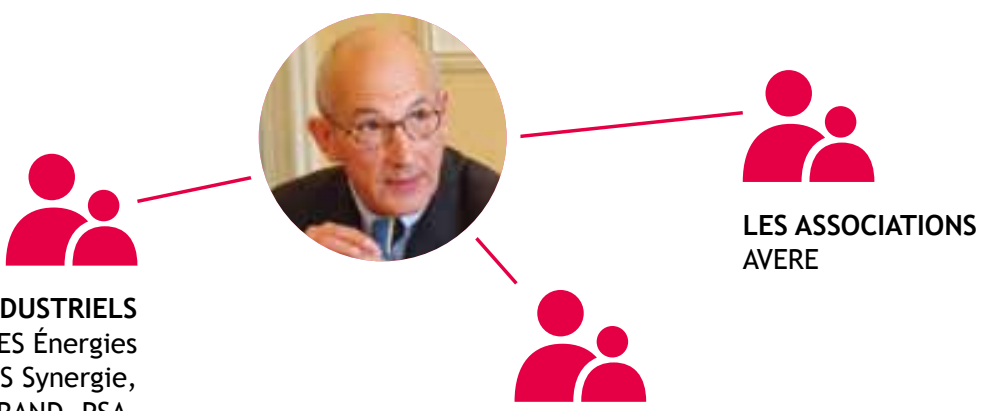

BOLLORÉ/IER, BOUYGUES Énergies Service, DBT-CEV, GIREVE, IES Synergie, LAFON Technologies, LEGRAND, PSA, RENAULT, SCHNEIDER ELECTRIC, EDF, ERDF, LA POSTE, SNCF

LES ACTEURS PUBLICS

MEIN, MEDDE, MLET, CGI, Bpifrance, ADEME

\section{SYNTHÈSE DES ACTIONS DU PLAN}

\section{Conditions industrielles du déploiement des bornes électriques de recharges}

Action

Calendrier

Préparation de la mise en œuvre du projet de directive définissant le standard européen de prises

2014

Mise à jour, sur la base d'une vision partagée, les perspectives du développement de l'électromobilité aux horizons 2015, 2020 et 2030

Mise en place d'un ensemble normatif et réglementaire pérenne suite aux évolutions techniques et réglementaires et aux premiers retours d'expériences

2014

Contribution, le plus en amont possible, à une définition des normes en matière d'interopérabilité compatibles avec les orientations européennes

Projets de déploiement des bornes électriques de recharge

Action

Soutien à la charge quotidienne de proximité (domicile, lieu de travail, parkings industriels et

commerciaux, lieux

recevant du public)

Prolongation, extension

et accentuation de

la dynamique de

déploiement public en

ville pour un maillage de

proximité

Réalisation d'un maillage national et ouvert

sur l'Europe

Calendrier

Engagements État

Premières mesures

en 2014 (loi ALUR et

Loi Programmation

Transition Énergétique)

Initiatives législatives, concertation

avec les parties prenantes sous

l'égide du Gouvernement, soutien

à l'innovation technologique et des usages (investissements d'avenir) pour accélérer les projets des collectivités locales dans le cadre des CPER et du Programme des de $50 \mathrm{M€}$ )

Négociations en 2014

Accompagnement de l'État dans
Engagements industriels

Offre technico-commerciale disponible et performante fabriquée en France, maîtrise des coûts des équipements et de l'installation, retour en activité industrielle et en emplois

Accompagnement des collectivités locales pour optimiser les plans de déploiement aux besoins Investissements d'Avenir (enveloppe

Contribution à la visibilité du réseau de recharge par l'équipement des voies de plus forte fréquentation (autoroutes) ses prérogatives régaliennes et en qualité d'investisseur avisé, le cas échéant

$$
\text { et } 2015
$$

Utilisation du levier des contrats d'objectif et des prérogatives de l'autorité concédante pour négocier avec les concessionnaires d'autoroutes et avec les exploitants des stations-service
Propositions de déploiement de réseau intelligent et connecté d'infrastructures de recharge pour véhicules électriques émanant des acteurs privés, ouverts à tous types et marques de véhicules électriques 


\section{BORNES ÉLECTRIQUES DE RECHARGE}
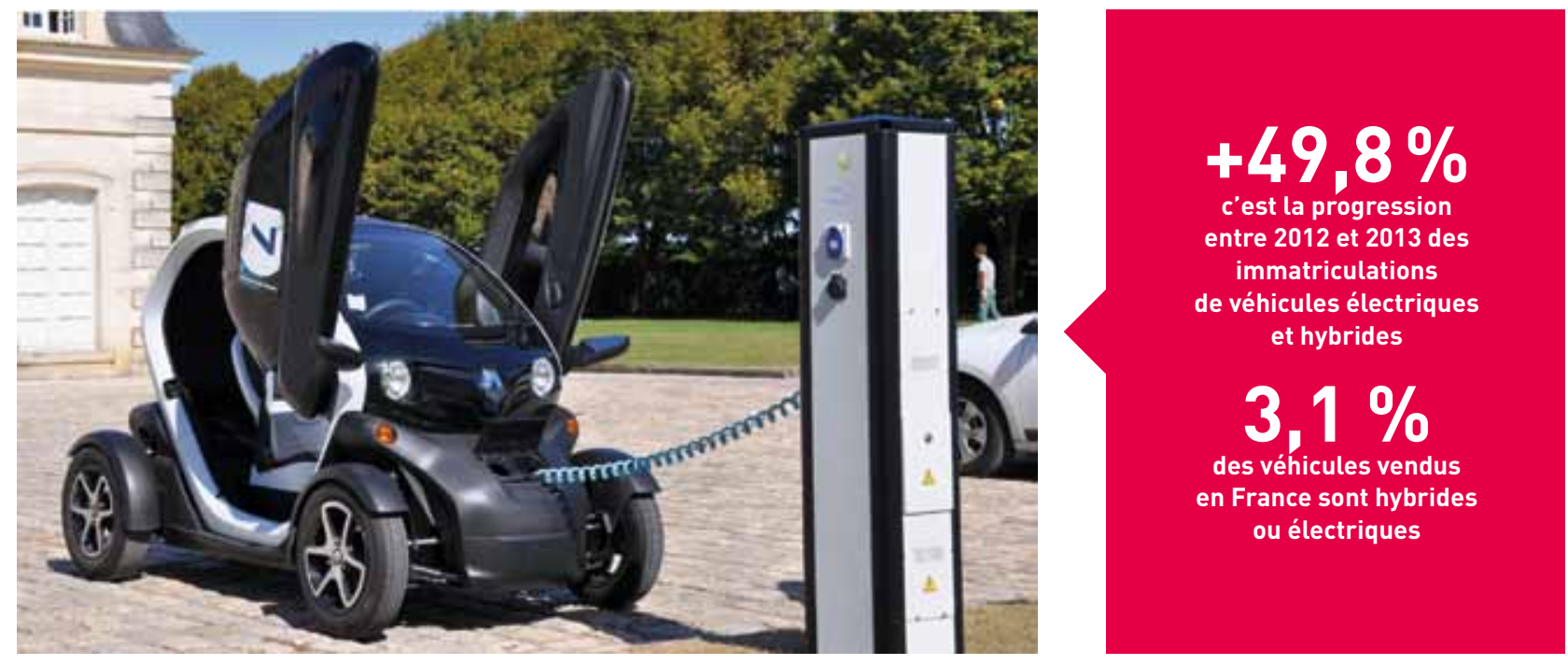

Borne de recharge électrique, Saintronic

Nous construisons la France de la mobilité électrique sans frontière. Levier indispensable à la réduction des émissions de gaz à effet de serre et des pollutions atmosphériques locales, l'électromobilité peut et doit être mise au service de notre souveraineté énergétique. Elle permet en effet de limiter la part des hydrocarbures importés dans notre consommation d'énergie. - Notre pays est le premier marché d'Europe pour les véhicules électriques et hybrides de nouvelle génération sur lesquels nos constructeurs ont pris une avance technologique reconnue et produisent des véhicules sur le territoire national. Au plan mondial, le marché cible est considérable et en Europe, les ventes ont été multipliées par 20 entre 2010 et 2012 . Le véhicule électrique constitue une innovation technologique et écologique majeure, ce qui explique son attractivité. Mais la nouveauté de ce type de véhicule suscite aussi des demandes chez les clients potentiels qui veulent pouvoir «faire le plein » facilement, chez eux, sur leur lieu de travail, partout et à toute heure. La question du rechargement est donc cruciale. - Le plan a pour vocation d'amplifier et de structurer le réseau de bornes de recharges électriques et de faire de la France la championne de la mobilité électrique. Il vise à amplifier les efforts partagés de l'État et des acteurs industriels qui produisent aussi sur le territoire français, en lien avec l'ensemble des parties prenantes, notamment les collectivités territoriales, en faveur des véhicules électriques (bonus écologique, commande de véhicules de l'État réorientée au minimum à $25 \%$ vers les véhicules électriques et hybrides, soutien massif à l'innovation au travers du programme des investissements d'avenir). L'État soutient également dans le cadre des investissements d'avenir les projets d'infrastructures de charge des collectivités locales. La France possède déjà le premier parc de points de charge ouverts (ou programmés) en Europe qui atteint les 8000 à la fin 2013 et il doit encore doubler en 2014. 
CHEF DE PROJET

Florence Lambert est directrice du laboratoire Innovation des Technologies de l'Énergie (LITEN) au Commissariat à l'Énergie atomique et aux énergies alternatives.

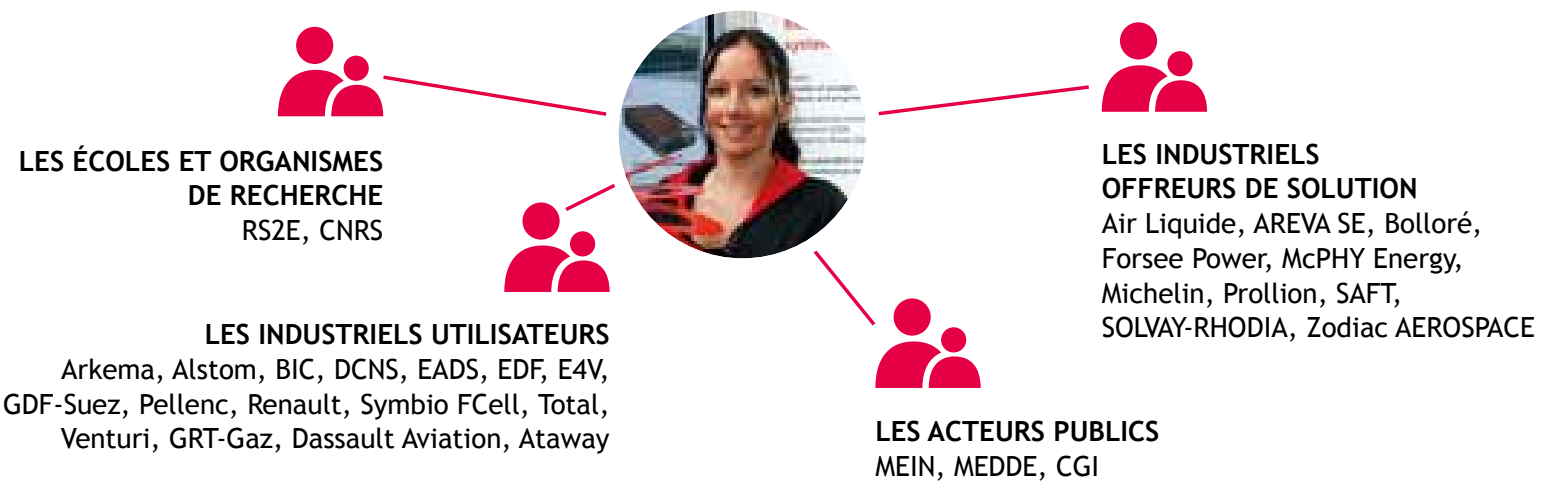

\section{SYNTHÈSE DES ACTIONS DU PLAN}

Batterie : consolider et structurer une filière complète de fabrication de batteries

\begin{tabular}{lll} 
Action & Calendrier & Finalité/Livrable \\
\hline Stockage des énergies intermittentes & $2015-2017$ & $\begin{array}{l}\text { Déploiement de capacités de stockage, progrès } \\
\text { dans les performances (interfaces de gestion de } \\
\text { l'énergie), confortation du tissu industriel en France }\end{array}$ \\
\hline $\begin{array}{l}\text { Émergence d'un chimiste français pour } \\
\text { l'approvisionnement des industriels de la batterie }\end{array}$ & $\begin{array}{l}\text { Phase pilote en 2015 } \\
\text { Production en 2016 }\end{array}$ & $\begin{array}{l}\text { Industrialisation en France (une à trois unités de } \\
\text { production d'intermédiaires chimiques en France) }\end{array}$ \\
\hline $\begin{array}{lll}\text { Filières de batteries Li-ion pour applications spécifiques } \\
\end{array}$ & $\begin{array}{l}\text { Phase pilote en 2015 } \\
\text { Production en 2017 }\end{array}$ & $\begin{array}{l}\text { Consortium de R\&D débouchant sur la } \\
\text { réalisation d'une ligne pilote puis d'unités } \\
\text { de production }\end{array}$ \\
\hline $\begin{array}{l}\text { Soutien à la filière d'assemblage en France } \\
\text { de cellules Li-ion }\end{array}$ & $\begin{array}{l}\text { Immédiat } \\
\text { Mise en place d'un label de batteries }\end{array}$
\end{tabular}

Hydrogène: intégrer et structurer une filière énergétique complète du vecteur hydrogène

\begin{tabular}{|c|c|c|}
\hline $\begin{array}{l}\text { Développement d'une offre de «stack» française } \\
\text { compétitive pour pile à combustible }\end{array}$ & $\begin{array}{l}\text { Premier produit livré } \\
\text { en } 2016\end{array}$ & $\begin{array}{l}\text { Investissement privé, potentiel } \\
\text { co-investissement de l'État }\end{array}$ \\
\hline $\begin{array}{l}\text { Aide au développement d'offres de stockage } \\
\text { d'hydrogène haute pression }\end{array}$ & 2015 & R\&D industrielle \\
\hline $\begin{array}{l}\text { Aide aux clients précurseurs (chariots élévateurs, VUL, } \\
\text { Bus urbains) }\end{array}$ & Première flotte en 2015 & $\begin{array}{l}\text { Démonstration d'usage via des flottes privées } \\
\text { vitrines }\end{array}$ \\
\hline $\begin{array}{l}\text { Définition d'un modèle économique pour } \\
\text { le déploiement d'infrastructures }\end{array}$ & 2015 & $\begin{array}{l}\text { Évaluation de l'opportunité d'un partenariat } \\
\text { franco-allemand }\end{array}$ \\
\hline Filière industrielle «Power to Gaz» & $\begin{array}{l}\text { Lancement dès } 2014 \text {, } \\
\text { développement } 2015\end{array}$ & Réalisation de pilotes industriels \\
\hline Stockage nomade de l'hydrogène & $\begin{array}{l}\text { Phase pilote en } 2016 \\
\text { Production en } 2019\end{array}$ & Lab Fab puis industrialisation \\
\hline $\begin{array}{l}\text { Lancement d'une plateforme d'information sur les } \\
\text { technologies de l'hydrogène à destination du grand public. }\end{array}$ & $\begin{array}{l}\text { Immédiat avec } \\
\text { concrétisation } 2015\end{array}$ & $\begin{array}{l}\text { Mise en commun des } 11 \text { projets existants, } \\
\text { réalisation d'un site Web }\end{array}$ \\
\hline
\end{tabular}

\section{Collaboration internationale}

\begin{tabular}{lll}
\hline $\begin{array}{l}\text { Batteries de grande capacité (par exemple technologie } \\
\text { sodium soufre) }\end{array}$ & 2014-2015 & $\begin{array}{l}\text { Mise en place de collaborations internationales } \\
\text { (par exemple avec le Japon) }\end{array}$ \\
\hline Volants à inertie & $2014-2015$ & $\begin{array}{l}\text { Mise en place de collaborations internationales } \\
\text { (par exemple avec l'Allemagne) }\end{array}$
\end{tabular}




\section{AUTONOMIE ET PUISSANCE DES BATTERIES}
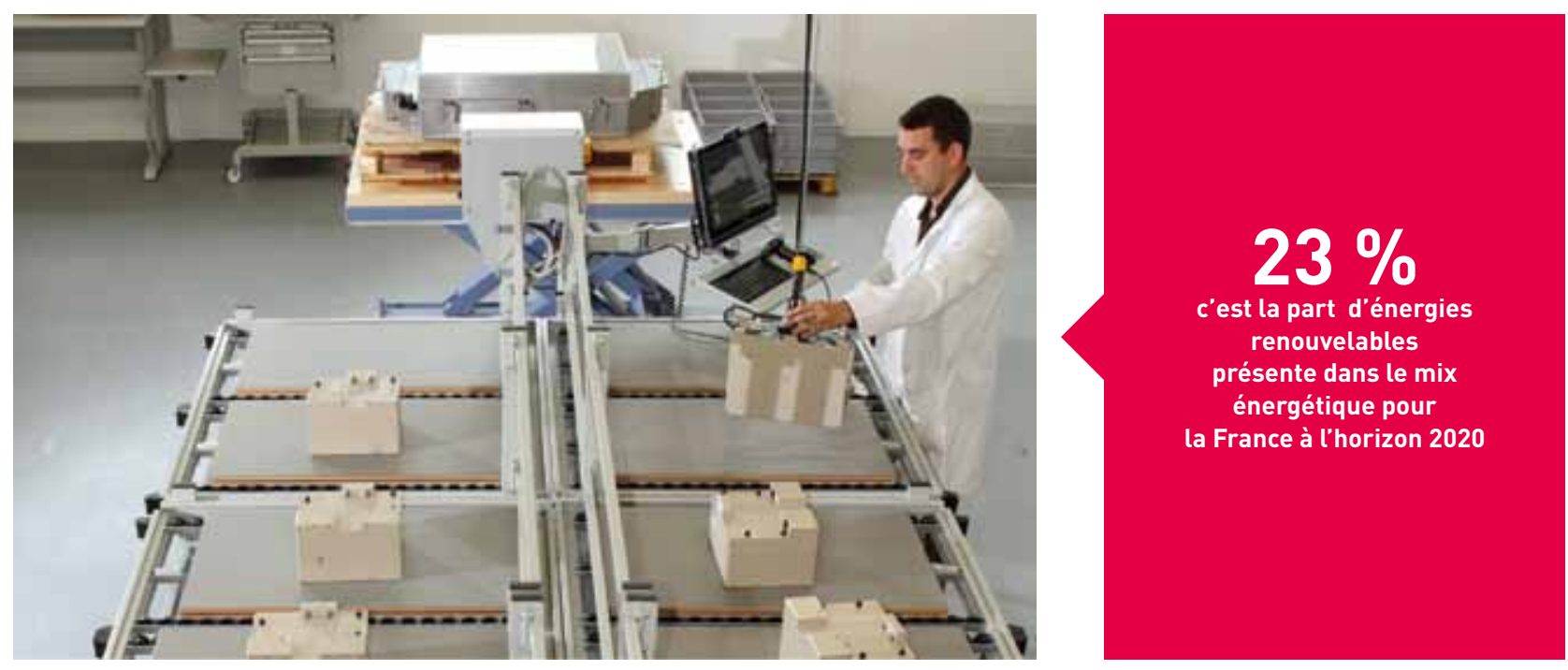

Usine de fabrication de batteries

- Nous construisons la France des batteries de longue durée et de la pile à hydrogène. Le stockage de l'énergie propre sera l'une des clés du succès de la transition énergétique, pour sécuriser des sites isolés, soutenir les réseaux, pallier l'intermittence des énergies renouvelables ou permettre une mobilité électrique décarbonnée. Le stockage électrique par voie hydrogène ou électrochimique (batteries) constitue deux filières parallèles mais complémentaires. - La batterie offre un très bon rendement sur de courtes à moyennes durées, alors que l'hydrogène associé à une pile à combustible peut répondre aux besoins de stockage flexible et de longue durée avec une charge rapide. Ensemble, ces technologies représentent potentiellement un chiffre d'affaires de 30 milliards d'euros annuel à l'horizon 2030. - La France dispose d'acteurs industriels dans ces deux filières pour les applications stockage des énergies renouvelables, transport, défense et aérospatial. - Le plan a permis de mobiliser des acteurs industriels de toutes tailles dans la poursuite d'objectifs communs pour l'intérêt économique des deux filières. - En matière de batteries, les entreprises françaises sont déjà bien positionnées sur les secteurs des batteries de spécialité à haute valeur ajoutée (Lithium-lon et Lithium métal polymère). Ce plan va permettre à la filière de se structurer en remontant la chaîne de valeur (intermédiaires chimiques produits en France) et en proposant des solutions industrielles sécurisées. - Pour que la filière hydrogène se structure, se consolide et devienne créatrice d'emplois et d'innovation en France, une véritable Équipe de France de l'hydrogène a été réunie par l'État. Le déploiement du plan permettra de franchir une nouvelle étape pour donner naissance à un acteur intégré de l'industrialisation de la filière en préservant une fiabilité et une sécurité maximales. - Enfin, il permettra la combinaison entre le stockage batterie et le vecteur hydrogène, améliorant ainsi l'autonomie des systèmes de stockage. La mise en œuvre d'une chaîne de traction hybridée pourra être le point d'excellence et de différenciation français. 


\section{ÉQUIPE DU PLAN}

CHEF DE PROJET

Carlos Ghosn est PDG du groupe Renault/Nissan.

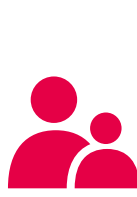

LES INDUSTRIELS

Acteurs concernés de la Plateforme de la Filière Automobile (Renault,

PSA, Valeo, Michelin), Deways, Iveco, Ligier, RATP, Renault Trucks, Robosoft, Sanef, UTAC, Vulog
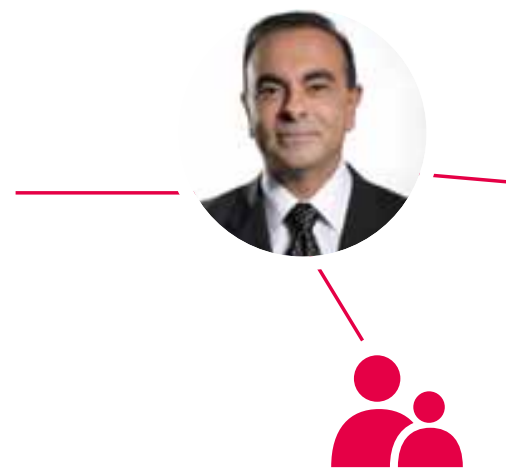

FOURNISSEURS DE TECHNOLOGIE

AJS-ID, Akka Technologies, All4Tec, Apojee, Assystem, AVL, Bertin Technologies, Car\&D, Continental France, Dassault Systèmes, Effidence, EcosysGroup, ESI Group, Esterel

Technologies, Intempora, IRT SystemX, ITE Vedecom, JCAE, LMS Imagine, M3 Systems, Magna Steyr France, Marben Products, Navecom, Nexyad, OKTAL, Spirops, StatXpert, STMicroelectronics, Tecris, TrustInSoft, Vici Consult, Vimades, Vulog

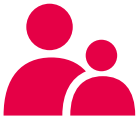

LES ACTEURS PUBLICS

MEIN, Intérieur, MEDDE, MEDDE, CGI, CEREMH, CEA LIST, CEESAR, ESIGELEC/ IRSEEM, GdR Robotique (CNRS), Heudiasyc, IBISC, ICST, IEF, IFSTTAR, Institut Pascal, INRIA, IRCCYN, IRTES, IRSTEA, ISIR, ISM, LAAS, Labex IMobS3, LAMIH-CNRS, LIMOS, LITIS - Rouen, LISV - UVSQ, Laboratoire PRISM, Mines Paritech, Telecom Bretagne, Telecom Paritech, Université Grenoble 1, ISAT Nevers, pôles de compétitivité ID4CAR, LUTB Transport \& Mobility Systems, Movéo, Systematic, Véhicule du futur, ViaMéca

\section{SYNTHĖSE DES ACTIONS DU PLAN}

Pilote

Calendrier Finalités/livrables

\begin{tabular}{|c|c|c|c|}
\hline \multicolumn{4}{|l|}{ Coordonner les initiatives sur le véhicule autonome } \\
\hline $\begin{array}{l}\text { Identification et coordination des initiatives } \\
\text { locales }\end{array}$ & DGCIS & 2015 & Zones pilotes pour le véhicule autonome \\
\hline $\begin{array}{l}\text { Organisation de coopérations ou d'échanges } \\
\text { internationaux }\end{array}$ & DGCIS & 2015 & Coopération avec la Chine et la Corée \\
\hline \multicolumn{4}{|c|}{ Démontrer les bénéfices socio-économiques, sécuritaires et l'acceptabilité du véhicule autonome } \\
\hline $\begin{array}{l}\text { Étude de l'impact socio-économique } \\
\text { et sécuritaire et de l'acceptabilité }\end{array}$ & $\begin{array}{l}\text { PFA, Renault } \\
\text { Trucks, RATP }\end{array}$ & $2015-2020$ & $\begin{array}{l}\text { Expérimentations, études d'impact et études d'acceptabilité pour les cas } \\
\text { d'usage ciblés }\end{array}$ \\
\hline \multicolumn{4}{|c|}{ Investir dans les domaines technologiques clés du véhicule autonome } \\
\hline $\begin{array}{l}\text { Lancement des projets de R\&D ciblés } \\
\text { dans le domaine de l'intelligence embarquée, } \\
\text { des IHMs, des facteurs humains et de la } \\
\text { connectivité }\end{array}$ & $\begin{array}{l}\text { ITE Vedecom, } \\
\text { MEIN }\end{array}$ & $\begin{array}{l}2014 \\
2015-18\end{array}$ & $\begin{array}{l}\text { Appel à projets dédié « Véhicule Autonome » (décembre) } \\
\text { Lancement et réalisation des projets R\&D }\end{array}$ \\
\hline Création et participation à des compétitions & DGCIS & $\begin{array}{l}2016 \\
2017 / 2018\end{array}$ & $\begin{array}{l}\text { Challenge technologique en France } \\
\text { Challenge technologique à l'international }\end{array}$ \\
\hline Encouragement de l'investissement des acteurs & DGCIS & $2015-2018$ & Interventions ciblées pour soutenir l'investissement des entreprises clés \\
\hline \multicolumn{4}{|c|}{ Démontrer l'amélioration de la sécurité par le véhicule autonome dans les cas d'usage ciblés } \\
\hline $\begin{array}{l}\text { Lancement de projets ciblés de R\&D } \\
\text { dans le domaine de la sécurité }\end{array}$ & $\begin{array}{l}\text { IRT SystemX, } \\
\text { MEIN }\end{array}$ & $\begin{array}{l}12 / 2014 \\
2015-18\end{array}$ & $\begin{array}{l}\text { IAppel à projets dédié «Véhicule Autonome» } \\
\text { Lancement et réalisation des projets R\&D }\end{array}$ \\
\hline $\begin{array}{l}\text { Mise à disposition des moyens d'essais } \\
\text { adaptés et démonstrations de l'amélioration } \\
\text { de la sécurité }\end{array}$ & $\begin{array}{l}\text { Gestionnaire } \\
\text { d'infrastructure } \\
\text { d'essais, } \\
\text { Constructeurs }\end{array}$ & $\begin{array}{l}2016 \\
2016-2018 \\
2015 \text { à } 2018\end{array}$ & $\begin{array}{l}\text { Centre d'essai urbain et péri-urbain } \\
\text { Zones de conduite autonome } \\
\text { Essais en situation réelle sur voie à chaussées séparées, puis sur sites urbains } \\
\text { et péri-urbains }\end{array}$ \\
\hline \multicolumn{4}{|c|}{ Faire évoluer le cadre réglementaire et normatif en vue de l'expérimentation puis de la mise sur le marché du véhicule autonome } \\
\hline $\begin{array}{l}\text { Évolution du cadre réglementaire } \\
\text { et normatif en vue de l'expérimentation } \\
\text { puis de la mise sur le marché }\end{array}$ & $\begin{array}{l}\text { Groupe inter- } \\
\text { administration } \\
\text { (DGEC, DGCIS, } \\
\text { DGTIM, DSCR, } \\
\text { DAJ MEDDE }\end{array}$ & $\begin{array}{l}12 / 2014 \\
2015 \\
2017 / 18 \\
2019\end{array}$ & $\begin{array}{l}\text { Texte réglementaire autorisant l'expérimentation sur route ouverte } \\
\text { Proposition de standards et de réglementations pour l'expérimentation } \\
\text { Label français «Véhicule Autonome Sûr» } \\
\text { Normes sur les processus et définition des standards des règles de tests }\end{array}$ \\
\hline $\begin{array}{l}\text { Adaptation des infrastructures en fonction } \\
\text { du contexte }\end{array}$ & DGITM & $\begin{array}{l}2018 \\
2020\end{array}$ & $\begin{array}{l}\text { Adaptation des infrastructures nécessaires, hors équipement de connectivité } \\
\text { Déploiement des équipements nécessaires de bords de route pour la } \\
\text { connectivité }\end{array}$ \\
\hline Mise en place d'un système d'assurance & MEIN & 2018 & Fonds d'assurance spécial véhicule autonome \\
\hline Évolution de la formation des conducteurs & DSCR & $2016 / 2017$ & Mise en place de supports de formation \\
\hline
\end{tabular}




\section{VÉHICULE AUTONOME}
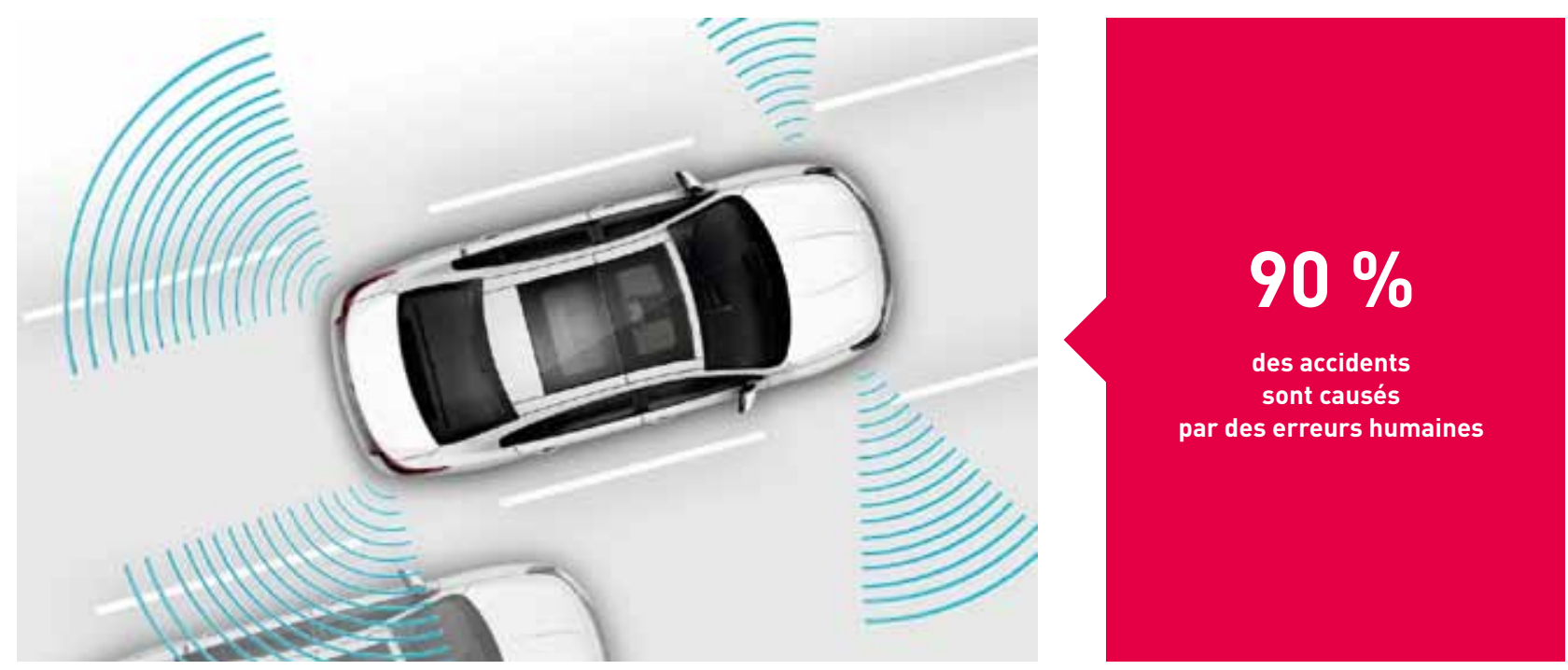

Perception de l'environnement

Nous construisons la France des véhicules intelligents et autonomes. Equipés de capteurs et de systèmes de commande innovants, ces véhicules contribuent à renforcer la sécurité routière et à dégager à terme pour le chauffeur, en toute sécurité, un nouveau temps libre ou productif. Ils permettent également de fluidifier le trafic, de développer de nouveaux services de transport public souples et adaptables en fonction des flux, et de rendre le transport plus accessible aux personnes en situation de handicap, aux personnes âgées et aux publics les plus vulnérables. - Ce véhicule, dont le développement est stratégique pour l'industrie et le maintien des emplois, s'appuie sur les avancées des aides à la conduite. Avec les grands acteurs de l'internet et du numérique, les filières de l'automobile et du transport développent une offre compétitive de composants, de capteurs, de logiciels, de systèmes de commande et de services afin de proposer, d'ici à 2020, des véhicules autonomes à prix abordable. L'ambition de ce programme est de faire de l'industrie française de l'automobile et du transport une des pionnières dans la conception des véhicules autonomes pour tous. - Le plan d'action prévoit d'approfondir l'étude de l'impact socio-économique de ces véhicules et de développer les technologies nécessaires, de permettre des expérimentations en toute sécurité afin de démontrer l'amélioration de la sécurité routière et de lever les obstacles réglementaires, sociaux et matériels à leur déploiement. Dans le cadre du plan, de premiers essais en route ouverte auront lieu dès 2015, en parallèle du lancement de projets de recherche et développement. - Avec ce plan, la France sera reconnue comme une terre d'expérimentations du véhicule autonome, un centre d'excellence de l'intelligence embarquée, et un leader en sécurité des systèmes complexes. Un marché de plusieurs milliards d'euros à l'échelle mondiale d'ici à dix ans s'ouvre à ceux qui sauront être les pionniers. 


\section{ÉQUIPE DU PLAN}

CHEF DE PROJET

Jean J. Botti est directeur général délégué Technologie et Innovation, membre du Comité Éxécutif d'AIRBUS GROUP et président de la Fondation d'entreprise d'AIRBUS GROUP.

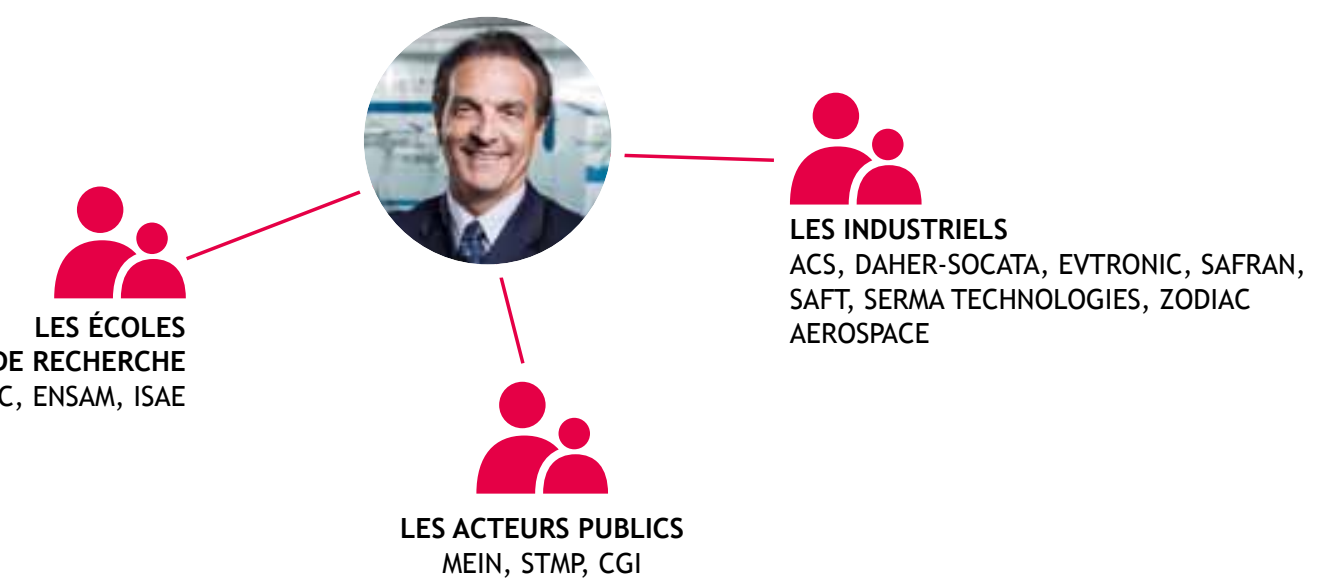

\section{SYNTHÈSE DES ACTIONS DU PLAN}

\begin{tabular}{|c|c|c|c|c|}
\hline Action & Pilote & Calendrier & $\begin{array}{l}\text { Acteurs publics } \\
\text { concernés }\end{array}$ & $\begin{array}{l}\text { Levier(s) public(s) } \\
\text { à actionner }\end{array}$ \\
\hline $\begin{array}{l}\text { Premiers vols techniques du } \\
\text { prototype expérimental e-Fan }\end{array}$ & AIRBUS GROUP, ACS & Janvier - Mars 2014 & DGAC & \\
\hline Vols de démonstrations & $\begin{array}{l}\text { AIRBUS GROUP, } \\
\text { DGAC }\end{array}$ & Courant 2014 & DGAC & \\
\hline $\begin{array}{l}\text { Étude préalable à une } \\
\text { phase d'avant-projet et de } \\
\text { levée de risques de l'e-Fan } \\
\text { commercialisé }\end{array}$ & AIRBUS GROUP & Printemps 2014 & $\begin{array}{l}\text { Écoles de pilotages, } \\
\text { ENAC }\end{array}$ & $\begin{array}{l}\text { Participation des écoles } \\
\text { de pilotages, ENAC }\end{array}$ \\
\hline \multicolumn{5}{|l|}{$\begin{array}{l}\text { Étude de marché pour design } \\
\text { de l'e-Fan commercialisé }\end{array}$} \\
\hline $\begin{array}{l}\text { Conception d'un projet } \\
\text { de développement } \\
\text { et d'industrialisation }\end{array}$ & AIRBUS GROUP & $\begin{array}{l}\text { Projet finalisé } \\
\text { avant la fin du } \\
\text { 3e trimestre } 2014\end{array}$ & $\begin{array}{l}\text { Régions, CGI, } \\
\text { DGAC, DGCIS, } \\
\text { écoles, ENAC }\end{array}$ & Investissements d'avenir \\
\hline $\begin{array}{l}\text { Mise en place d'un site } \\
\text { de production à Bordeaux }\end{array}$ & AIRBUS GROUP & $\begin{array}{l}\text { Second semestre } \\
2016\end{array}$ & $\begin{array}{l}\text { Région Aquitaine, } \\
\text { écoles }\end{array}$ & Soutien régional \\
\hline $\begin{array}{l}\text { Concertation des feuilles } \\
\text { de route des plans «Avion } \\
\text { électrique» et «Autonomie } \\
\text { et puissance des batteries» }\end{array}$ & CEA, AIRBUS GROUP & $\begin{array}{l}\text { Au rythme des } \\
\text { Comités de pilotage } \\
\text { NFI }\end{array}$ & CGI, DGCIS & \\
\hline $\begin{array}{l}\text { Alignement des jalons innovants } \\
\text { de l'e-Fan avec la composante } \\
\text { électrique de la feuille de route } \\
\text { CORAC }\end{array}$ & $\begin{array}{l}\text { Comité de pilotage } \\
\text { CORAC }\end{array}$ & & $\begin{array}{l}\text { DGAC, DGA, DGCIS, } \\
\text { MENESR }\end{array}$ & \\
\hline
\end{tabular}




\section{AVION ÉLECTRIQUE ET NOUVELLE GÉNÉRATION D'AÉRONEFS}
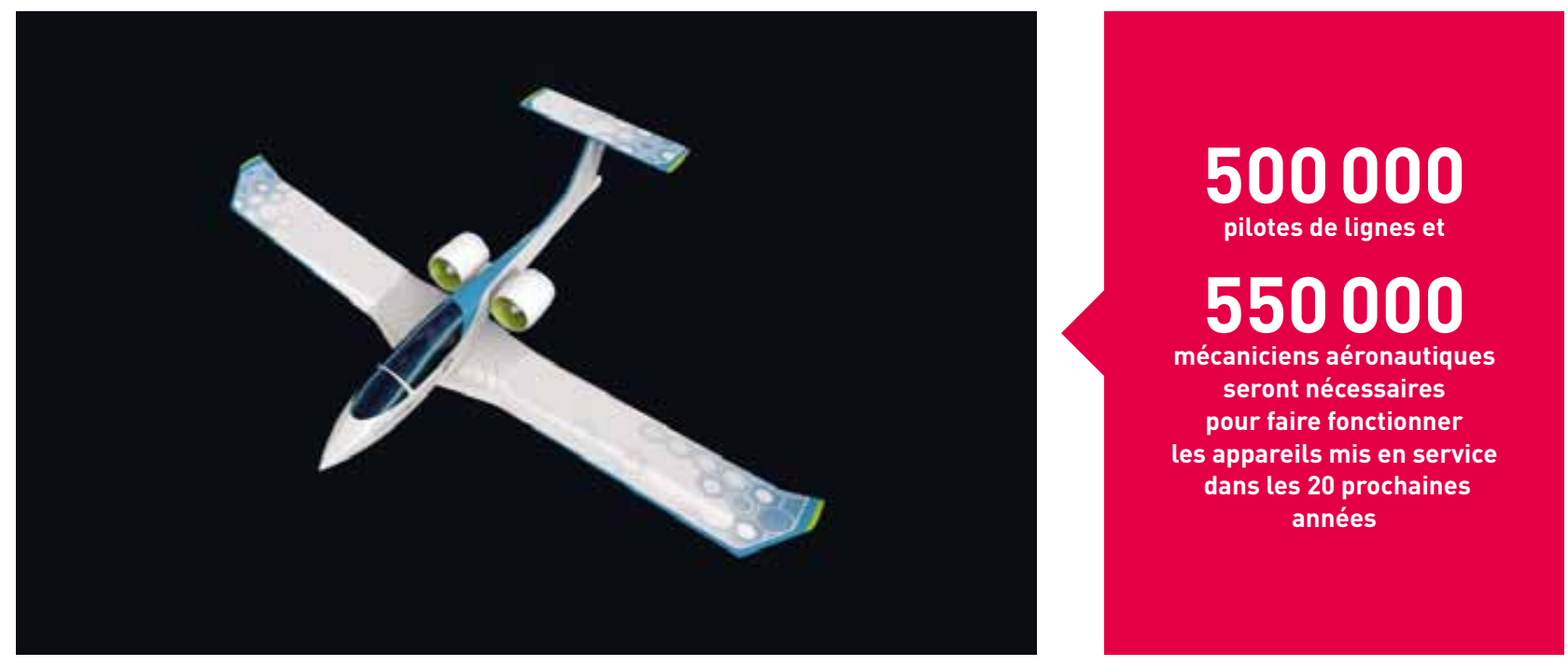

Avion électrique biplace, EADS Innovative Works

- Nous construisons la France championne de l'industrie aéronautique. À horizon 2030 dans le monde, plusieurs dizaines de milliers d'avions nouveaux devraient être mis en service, générant un besoin de formation de 500000 pilotes de ligne. La France doit saisir cette opportunité de promouvoir son savoir-faire industriel et de formation. Notre pays est en mesure de porter la rupture technologique dont le monde de l'aviation a besoin pour faire face à cette croissance dans le contexte de la nécessaire transition énergétique. - Le plan pose les bases d'une aviation commerciale à motorisation hybride et électrique dans une vision long terme. Il doit permettre aux acteurs de la filière aéronautique nationale de monter en compétence sur les technologies associées, notamment le stockage et la gestion de l'énergie électrique à bord, en vue de leur application progressive à des avions de taille toujours plus grande. Il est structuré autour d'un projet central d'avion-école biplace tout électrique, l'«e-Fan», qui répond à l'enjeu d'un développement de la formation au pilotage compatible avec les exigences environnementales et contribue à la maturité des technologies applicables aux avions de ligne toujours plus électriques. - Le développement et l'industrialisation de l'e-Fan, aujourd'hui à l'état de prototype, doivent par ailleurs permettre de faire émerger en France une nouvelle filière capable de prendre des positions fortes sur le marché des avions légers dédiés à la formation initiale des pilotes. L'État a veillé à la mise en relation les acteurs autour d'Airbus Group, qui s'est rapproché de partenaires industriels, d'organismes de recherche et d'écoles de formation pour constituer un consortium rassemblant les compétences nécessaires à la levée des verrous techniques et à la mise sur le marché de l'e-Fan à horizon de 4 ou 5 ans. Le pilotage de ce projet sera porté par une filiale d'Airbus Group dédiée à l'aviation électrique Made in France, VoltAir. Le projet débouchera sur la construction en Aquitaine d'une usine dédiée à l'assemblage de l'e-Fan, fondée sur une production en partenariat avec les PME locales. 


\section{ÉQUIPE DU PLAN}

\section{CHEFS DE PROJET}

Jean-Yves Longère est directeur général du pôle de compétitivité Pégase.

Bruno Even est directeur général de SAGEM.

Francis Durufle est vice-président de la Fédération Professionnelle du Drône Civil.

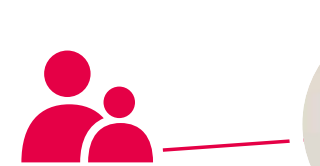

OPÉRATEURS
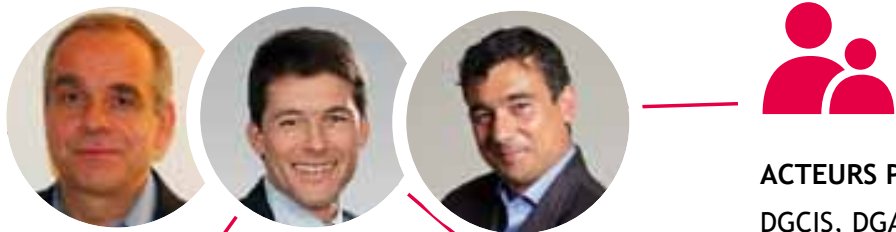

\section{ACTEURS PUBLICS}

DGCIS, DGAC, DGA et les pôles de compétitivité aéronautique AEROSPACE VALLEY, ASTECH, PEGASE, SYSTEMATIC..

Volet dirigeables: Airship Vision, Airship Paris...

Volet drones civils: AirMarine, Azur Drones, Delta Drone, Diades, Exametrics, L'Avion Jaune, RedBird...

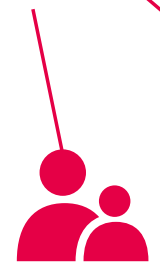

UTILISATEURS «DONNEURS D'ORDRES»

Volet dirigeables: opérateurs des secteurs offshore, logistique, BTP, bois, télécoms, énergie...

Volet drones civils: SNCF, EDF, GRTgaz, Bouygues Energie et Services...
ÉCOLES ET ORGANISMES

DE RECHERCHE ONERA,

CEA, CNES, École de l'Air, ENAC, IRSTEA...

\section{LES INDUSTRIELS}

Volet dirigeables: Airstar, A-NSE, Euro Airship, Ciel, Flying Whales, Thales Alenia Space, Voliris, Z Marine, Zodiac Aerospace...

Volet drones civils: Airbus Group, Sagem, Thales, Gorgé-Infotron, Parrot, Delair Tech, Novadem, Fly'n Sense..

\section{SYNTHĖSE DES ACTIONS DU PLAN}

\begin{tabular}{|c|c|c|c|c|}
\hline Action & Calendrier & Acteurs privés & Acteurs publics & Finalités/livrables \\
\hline \multicolumn{5}{|l|}{ Volet dirigeables } \\
\hline $\begin{array}{l}\text { Labellisation des projets, sur la base } \\
\text { notamment d'une analyse des coûts } \\
\text { et des plans de financement }\end{array}$ & $\begin{array}{l}\text { T3 } 2014 \text { (ouverture } \\
\text { de la procédure) }\end{array}$ & $\begin{array}{l}\text { Porteurs de projet, pôles } \\
\text { de compétitivité, experts } \\
\text { indépendants }\end{array}$ & $\begin{array}{l}\text { ONERA, collectivités } \\
\text { territoriales }\end{array}$ & $\begin{array}{l}\text { Connaissance et évaluation } \\
\text { des projets existants } \\
\text { Recommandations aux } \\
\text { porteurs }\end{array}$ \\
\hline $\begin{array}{l}\text { Etudes de marché et développement } \\
\text { des usages }\end{array}$ & $\begin{array}{l}\text { A partir de fin } 2014 \\
\text { (mise en place d'un cadre } \\
\text { d'échanges) }\end{array}$ & $\begin{array}{l}\text { Utilisateurs finaux, } \\
\text { offreurs de solutions, } \\
\text { industriels, pôles de } \\
\text { compétitivité }\end{array}$ & $\begin{array}{l}\text { Ministères, collectivités } \\
\text { territoriales }\end{array}$ & $\begin{array}{l}\text { Validation des marchés } \\
\text { Analyse de cas d'usages } \\
\text { Spécifications des solutions } \\
\text { à base de dirigeables }\end{array}$ \\
\hline $\begin{array}{l}\text { Levée des verrous technologiques } \\
\text { et réglementaires }\end{array}$ & $\begin{array}{l}2015 \text { (lancement d'un } \\
\text { programme de recherche } \\
\text { collaborative) }\end{array}$ & $\begin{array}{l}\text { Industriels, acteurs } \\
\text { technologiques, pôles de } \\
\text { compétitivité }\end{array}$ & $\begin{array}{l}\text { Écoles d'ingénieurs, } \\
\text { laboratoires de recherche, } \\
\text { ministères } \\
\text { AESA }\end{array}$ & $\begin{array}{l}\text { Assurer la performance } \\
\text { opérationnelle des solutions } \\
\text { dirigeables }\end{array}$ \\
\hline $\begin{array}{l}\text { Etude, réalisation et essais } \\
\text { d'un prototype de dirigeable } \\
\text { charges lourdes }\end{array}$ & $\begin{array}{l}\text { Essais en vol } 5 \text { ans après le } \\
\text { lancement du programme }\end{array}$ & $\begin{array}{l}\text { Porteur(s) de projet et } \\
\text { partenaires industriels, } \\
\text { pôle Pégase }\end{array}$ & ONERA, DGA-EV, ministères & $\begin{array}{l}\text { Industrialisation d'une } \\
\text { filière dirigeables - charges } \\
\text { lourdes }\end{array}$ \\
\hline $\begin{array}{l}\text { Etude, réalisation et essais } \\
\text { du démonstrateur StratoBus }\end{array}$ & $\begin{array}{l}\text { Essais en vol } 6 \text { ans après le } \\
\text { lancement du programme }\end{array}$ & $\begin{array}{l}\text { Thales Alenia Space, } \\
\text { Z Marine, partenaires } \\
\text { avionique et motorisation, } \\
\text { pôle Pégase }\end{array}$ & $\begin{array}{l}\text { CEA, ONERA, DGA-EV, CNES, } \\
\text { ministères }\end{array}$ & $\begin{array}{l}\text { Industrialisation d'une } \\
\text { filière plateformes } \\
\text { stratosphériques }\end{array}$ \\
\hline \multicolumn{5}{|l|}{ Volet drones civils } \\
\hline Structuration de la filière & $\begin{array}{l}2014: \text { lancement à } \\
\text { l'automne }\end{array}$ & $\begin{array}{l}\text { FPDC, industriels, } \\
\text { donneurs d'ordres } \\
\text { et clients }\end{array}$ & DGAC, DGCIS & \\
\hline $\begin{array}{l}\text { Développement des briques } \\
\text { technologiques « certifiables " } \\
\text { dans un cadre réglementaire adapté }\end{array}$ & $2014-2018$ & $\begin{array}{l}\text { Constructeurs et } \\
\text { équipementiers de } \\
\text { l'avionique }\end{array}$ & DGAC, DGCIS & $\begin{array}{l}\text { Démonstrateurs } \\
\text { technologiques }\end{array}$ \\
\hline $\begin{array}{l}\text { Développement des équipements } \\
\text { de mission }\end{array}$ & $2015-2018$ & $\begin{array}{l}\text { Pôles de compétitivité, } \\
\text { tous acteurs industriels }\end{array}$ & DGCIS & $\begin{array}{l}\text { Projets industriels } \\
\text { et démonstrateurs }\end{array}$ \\
\hline Industrialisation & $2015-2018$ & Constructeurs & DGCIS, BPI & $\begin{array}{l}\text { Usine du futur } \\
\text { Plates-formes mutualisées }\end{array}$ \\
\hline $\begin{array}{l}\text { Soutien à l'export et promotion } \\
\text { de la filière }\end{array}$ & 2017 & $\begin{array}{l}\text { FPDC, Technowest, } \\
\text { pôles de compétitivité }\end{array}$ & $\begin{array}{l}\text { DGCIS, DGAC, UBI France, } \\
\text { BPI France }\end{array}$ & $\begin{array}{l}\text { Salon de renommée } \\
\text { internationale }\end{array}$ \\
\hline
\end{tabular}




\section{DIRIGEABLES ET DRONES CIVILS}
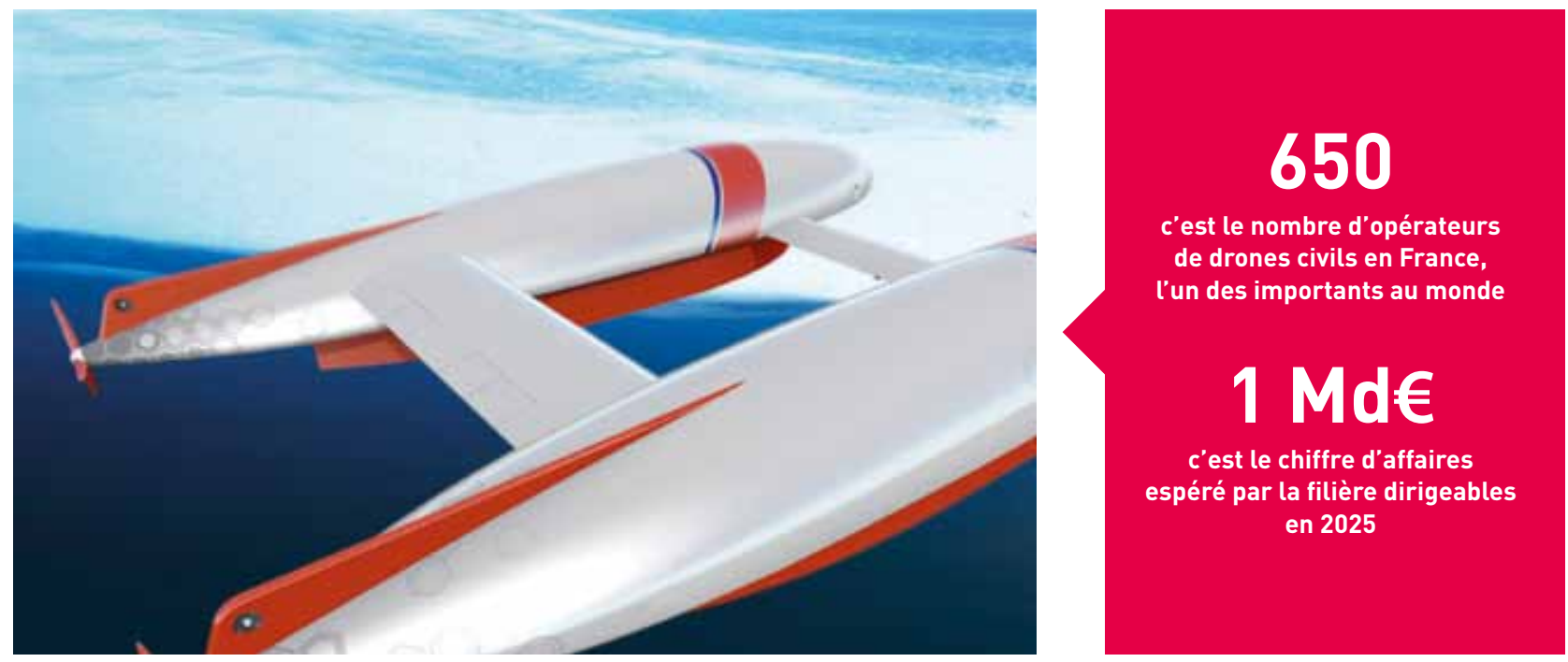

Esquisse de dirigeable-drone

- Nous construisons la France des futures filières aéronautiques. Les progrès techniques considérables réalisés depuis 50 ans par l'industrie aérospatiale permettent aujourd'hui d'envisager de nombreuses applications nouvelles pour les dirigeables. La France dispose pour ce faire de nombreux atouts : un tissu dynamique de start-up innovantes, des acteurs académiques de renommée internationale et une filière aérospatiale structurée autour de leaders mondiaux. Ces perspectives prometteuses ne doivent cependant pas masquer la complexité des défis qui restent à relever. - Le plan, pour son volet Dirigeables - charges lourdes, vise à coordonner les efforts de tous les acteurs pour combler les insuffisances opérationnelles des dirigeables et répondre aux exigences de sécurité aérienne. Grâce au développement d'un écosystème adapté, l'objectif est d'aboutir d'ici 2020 à la mise en service d'un prototype certifié de dirigeable dédié au transport point à point de charges lourdes, ainsi qu'à la validation par des essais en vol d'un démonstrateur de plateforme stratosphérique dédiée à des missions de surveillance et de télécommunication. • L'ambition du plan est également de permettre l'essor de l'utilisation des drones dans le domaine civil. La France a été parmi les premiers pays à se doter d'une réglementation spécifique pour favoriser l'émergence rapide d'un marché riche de plusieurs centaines de PME innovantes. Toutefois, une part importante des nouveaux services aériens qui pourraient être proposés ne pourront être rendus que par des drones plus lourds, plus performants et plus autonomes dans le respect des exigences en matière de sécurité des biens et des personnes. $\bullet$ Le plan, pour son volet Drones civils a vocation à lever les verrous actuels pour développer une filière industrielle qui servira une multitude de besoins dans l'agriculture, les réseaux, ou la protection de l'environnement. Cette diversité impose un modèle industriel réactif et à haute valeur ajoutée qui s'inscrit dans la réflexion de l'Usine du futur et qui garantit l'ancrage national des emplois liés au développement de cette activité. 


\section{ÉQUIPE DU PLAN}

CHEF DE PROJET

Éric Bantegnie est PDG d'Esterel Technologies.

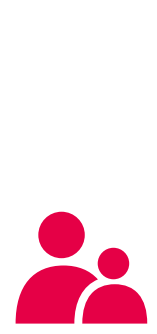

LES ÉCOLES ET ORGANISMES DE RECHERCHE CEA-List

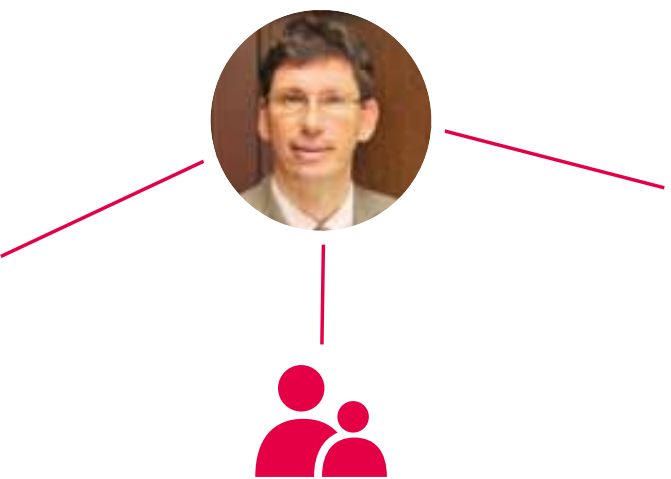

LES ACTEURS PUBLICS

Bpifrance, CGI, MEIN, DGCIS, MENESR

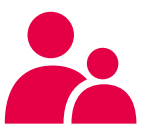

LES INDUSTRIELS

Actia, Airbus, Alstom, Altran, Renault, Safran, Schneider Electric, ST Microelectronics, Thales, Valeo

\section{SYNTHĖSE DES ACTIONS DU PLAN}

\begin{tabular}{|c|c|c|}
\hline Action & Calendrier & Finalité/Livrables \\
\hline \multicolumn{3}{|l|}{ Maîtrise de technologies critiques } \\
\hline $\begin{array}{l}\text { Conception d'une offre française } \\
\text { de plateforme d'exécution } \\
\text { pour applications industrielles } \\
\text { («Android pour applications } \\
\text { industrielles») }\end{array}$ & $\begin{array}{l}\text { Projet de R\&D de } 5 \text { ans : } \\
\text { - } 2014 \text { : lancement du projet de } \\
\text { plateforme et d'un cas d'usage } \\
\text { - } 2015 / 16 \text { : lancement de deux } \\
\text { nouveaux cas d'usage }\end{array}$ & $\begin{array}{l}\text { Le marché des systèmes embarqués est structuré par les plateformes } \\
\text { d'exécution disposant des bases installées les plus importantes. Ces } \\
\text { plateformes imposent ainsi des standards de fait. Il est donc critique } \\
\text { de positionner une plateforme française parmi les leaders du marché. Cette } \\
\text { action du plan vise à développer et à pérenniser une filière française dans } \\
\text { le domaine des plateformes d'exécution pour applications industrielles, en } \\
\text { s'appuyant sur les expertises réparties chez les acteurs dont dispose la France. }\end{array}$ \\
\hline $\begin{array}{l}\text { Maîtrise des technologies «multi- } \\
\text { cœurs» (processeur composé de } \\
\text { plusieurs unités de calcul) }\end{array}$ & $\begin{array}{l}\text { Projet de R\&D de } 5 \text { ans : } \\
\text { - Financement sur } 3 \text { ans de } \\
\text { projet de R\&D }\end{array}$ & $\begin{array}{l}\text { L'émergence de nouvelles architectures multi-cœurs laisse entrevoir } \\
\text { des gains spectaculaires en termes de performances de calcul et de } \\
\text { performances énergétiques. Ces architectures sont synonymes de défis } \\
\text { significatifs en matière de programmation. La présente action vise } \\
\text { à développer et pérenniser une filière industrielle dans le domaine } \\
\text { des technologies multi-cœurs. }\end{array}$ \\
\hline $\begin{array}{l}\text { Maîtrise de la simulation des } \\
\text { systèmes «cyber-physiques" } \\
\text { (systèmes regroupant des } \\
\text { composants électroniques } \\
\text { embarqués et des composants } \\
\text { physiques qu'ils contrôlent, ou } \\
\text { avec lesquels ils interagissent) }\end{array}$ & $\begin{array}{l}2015 \text { : prise en compte de cet } \\
\text { axe thématique dans des appels } \\
\text { à projets }\end{array}$ & $\begin{array}{l}\text { La simulation d'un système «cyber-physique» est extrêmement complexe du } \\
\text { fait des connaissances pluridisciplinaires qu'elle exige. Elle devient pourtant } \\
\text { une étape obligée de conception, pour garantir un fonctionnement et des } \\
\text { performances conformes aux attentes, tout en maîtrisant les coûts et les délais } \\
\text { de développement. La présente action vise à contribuer au développement } \\
\text { d'outils et de méthodologies ad hoc. }\end{array}$ \\
\hline \multicolumn{3}{|l|}{ Structuration de l'écosystème } \\
\hline $\begin{array}{l}\text { Mobilisation des acteurs } \\
\text { au sein de l'association } \\
\text { «Embedded France» }\end{array}$ & Dès 2014 & $\begin{array}{l}\text { La présente action vise à promouvoir une orientation des fonds de capital- } \\
\text { risque et de capital-développement vers les acteurs du secteur de l'embarqué, } \\
\text { et à défendre les intérêts communs de ces acteurs au sein de l'association } \\
\text { professionnelle «Embedded France» récemment fondée par Syntec Numérique, } \\
\text { Cap'tronic, ainsi que quatre pôles de compétitivité. }\end{array}$ \\
\hline \multicolumn{3}{|l|}{ Soutien aux actions de R\&D } \\
\hline $\begin{array}{l}\text { Soutien à des projets de R\&D } \\
\text { dans un cadre collaboratif }\end{array}$ & $\begin{array}{l}2014: \text { soutien de projets sur } \\
\text { la thématique de l'embarqué } \\
\text { et des objets connectés } \\
2015: \text { identification par } \\
\text { «Embedded France» de projets } \\
\text { particulièrement innovants } \\
\text { et orientation vers des guichets } \\
\text { de financement appropriés. }\end{array}$ & $\begin{array}{l}\text { - L'embarqué constitue un secteur à très forte intensité technologique où les } \\
\text { investissements en R\&D constituent une condition sine qua non de croissance. } \\
\text { La présente action vise à poursuivre les efforts de soutien à l'innovation } \\
\text { entamés dans le cadre du Fonds national pour la Société Numérique (FSN) } \\
\text { du Programme d'Investissements d'Avenir (PIA), avec une concentration } \\
\text { sur les technologies de l'embarqué et des objets connectés. } \\
\text { - } 16 \text { projets répondant à ces critères ont déjà été lancés dans le cadre } \\
\text { des Investissements d'avenir. }\end{array}$ \\
\hline
\end{tabular}




\section{LOGICIELS \\ ET SYSTĖMES EMBARQUÉS}
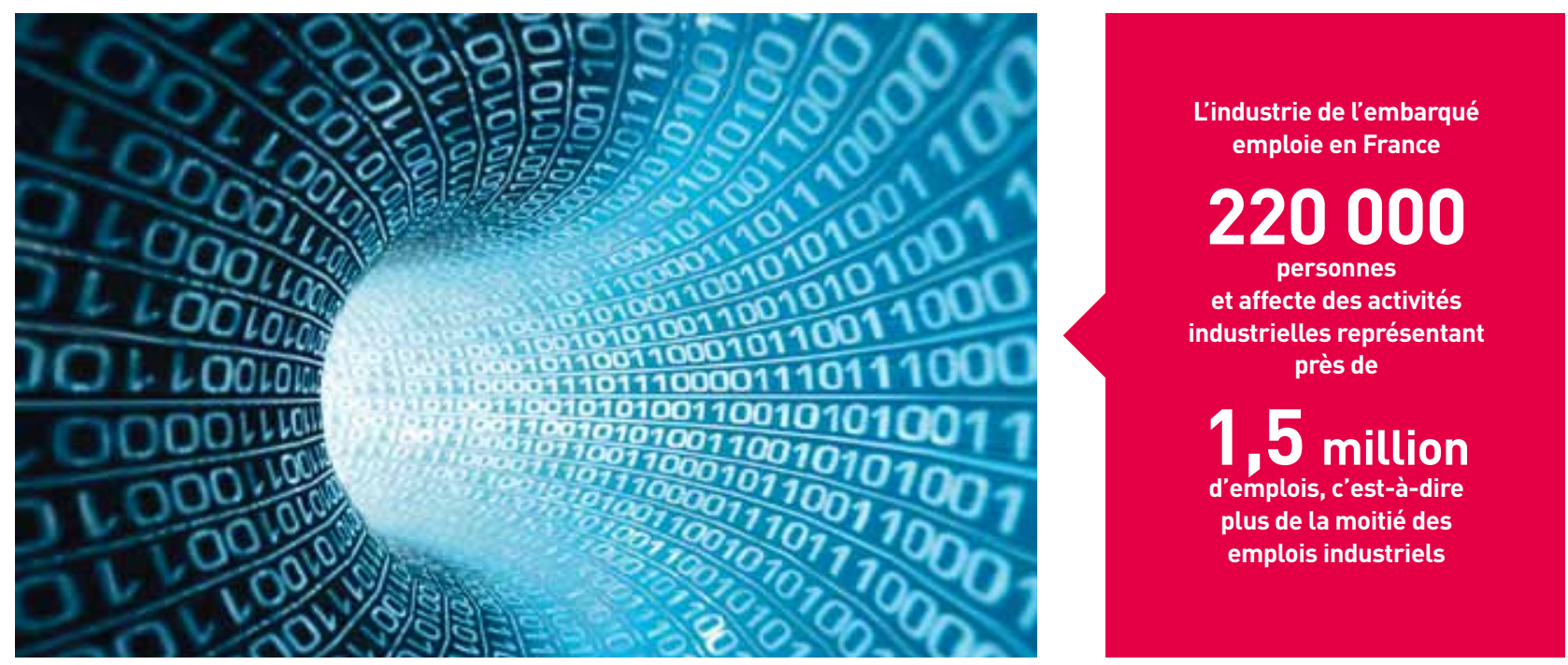

Time code it

- Nous construisons la France de l'intelligence des objets et des systèmes. - Les logiciels et systèmes embarqués sont partout dans notre environnement : smartphones, tablettes, boxes Internet, systèmes domotiques, dispositifs médicaux, automobiles, trains, avions... La maîtrise des technologies utilisées dans les logiciels et systèmes embarqués est fondamentale pour la compétitivité de nombreux secteurs industriels. Elle est essentielle pour la souveraineté numérique de notre pays. - Le marché mondial du logiciel embarqué atteint aujourd'hui 130 milliards d'euros, avec une croissance annuelle de l'ordre de $5 \%$. La France y occupe une part significative, avec un chiffre d'affaires d'environ 10 milliards d'euros. - L'industrie française de «l'embarqué » emploie plus de 220000 personnes et affecte des activités industrielles représentant près de 1 500000 emplois, c'est-à-dire plus de la moitié des emplois industriels existants. Cette industrie représente un puissant vecteur d'innovation et joue un rôle central dans les transitions énergétique et numérique de notre société. - L'élaboration du plan a associé à la fois des fournisseurs de technologies - essentiellement des PME et des ETI - et de grands groupes utilisateurs. - Les actions proposées poursuivent trois objectifs : la maîtrise de technologiques critiques pour la conception de systèmes embarqués, le soutien aux actions de R\&D dans un cadre favorisant la coopération entre fournisseurs de technologies et donneurs d'ordre, et la structuration d'un écosystème propice au développement des entreprises du secteur. - Compte tenu du caractère fortement diffusant des technologies en cause, les résultats du plan «logiciels et systèmes embarqués» pourront s'étendre dans de nombreux autres plans, tels les plans «voitures pour tous consommant moins de 2 litres aux $100 \mathrm{~km}$ », « robotique», ou «objets connectés». - La diffusion des technologies de l'embarqué constitue un outil de reconquête industrielle au service de la nouvelle France industrielle. 


\title{
ÉQUIPE DU PLAN
}

\author{
CHEF DE PROJET
}

Jean-Yves Le Gall est président

du Centre National d'Études Spatiales.

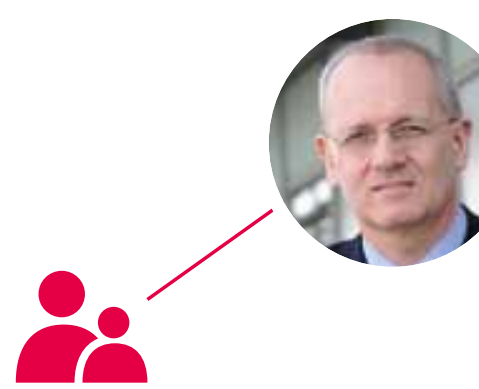

LES ACTEURS PUBLICS

CGI, MENESR, MEIN

\section{SYNTHÈSE DES ACTIONS DU PLAN}

Maître d'œuvre industriel

Airbus Defence \& Space,

1. Adaptation des plateformes géostationnaires à l'utilisation de

la propulsion électrique pour la mise

et le maintien à poste sur orbite

2. Développement du propulseur électrique de forte puissance

PPS 5000
Acteurs publics concernés et financement

CGI, MENESR, MINDEF

Financement de $25 \mathrm{M€}$ par

les investissements d'avenir sur un besoin total de $54 \mathrm{M€}$ 


\section{LE SATELLITE À PROPULSION ÉLECTRIQUE}
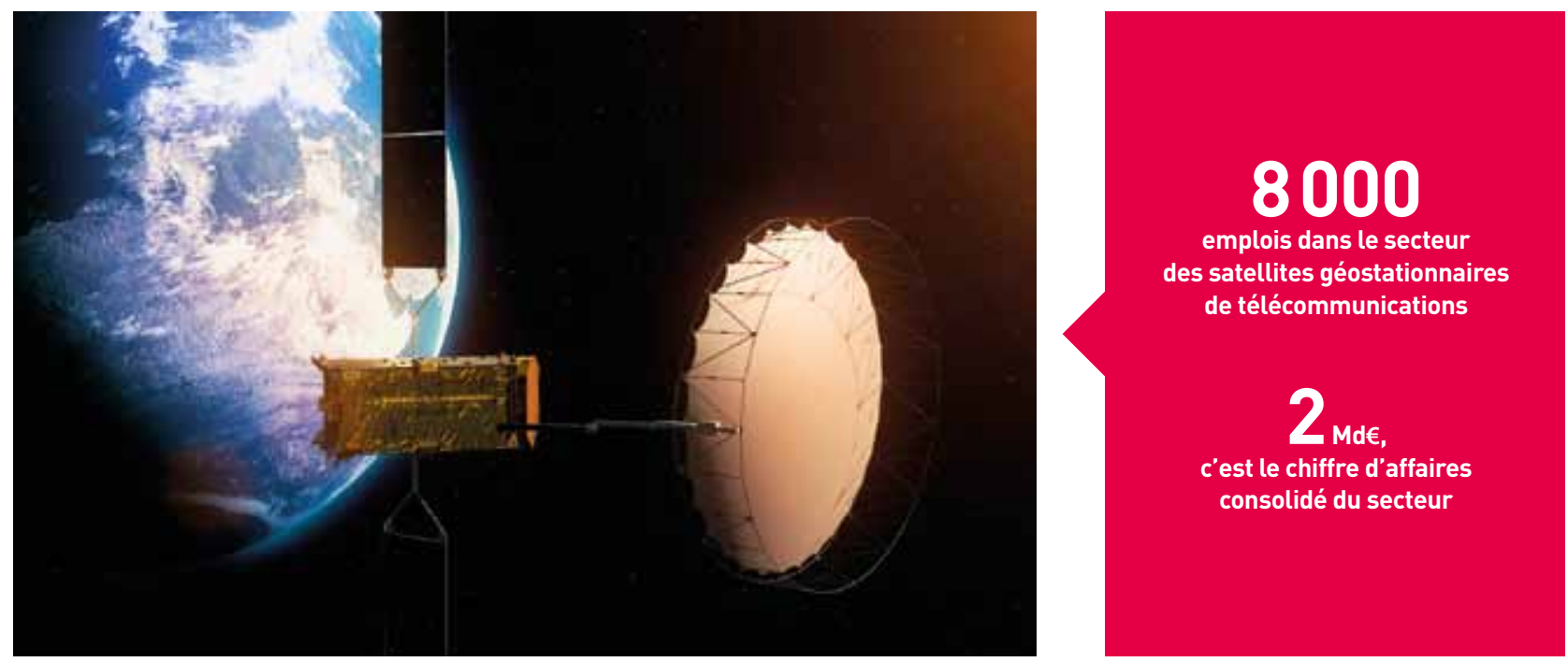

Esquisse d’un satellite à propulsion électrique

Nous construisons la France des satellites à propulsion électrique. On estime qu'à l'horizon 2020, le marché des satellites de télécommunications sera partagé entre satellites à propulsion chimique traditionnelle et satellites à propulsion électrique ou hybrides, la part de ces derniers ayant encore vocation à croître fortement au cours de la décennie suivante. - La France, qui excelle dans le domaine des satellites géostationnaires de télécommunications (ils représentent près de 8000 emplois sur notre territoire, 2 milliards d'euros de chiffre d'affaires consolidé, ainsi que de fortes perspectives de marché, en particulier à l'export), doit acquérir la pleine maîtrise de la technologie de la propulsion électrique. - Le plan a pour objectif de repositionner les fabricants français en tant que leaders mondiaux. Mis en œuvre par le CNES au nom de l'État, il est structuré autour de deux actions majeures. La première a pour objet de permettre l'adaptation des plates-formes de satellites géostationnaires des deux fabricants, Airbus et Thales, à l'utilisation de la propulsion électrique pour la mise et le maintien en orbite. Ces projets devront permettre la vente de satellites à propulsion électrique dès 2015 et le premier lancement d'un satellite de ce type dès 2017. La deuxième action vise à développer le propulseur électrique de forte puissance PPS 5000 de Snecma. L'industrie française devra disposer d'un propulseur qualifié pour sa configuration d'usage la plus exigeante en 2017 pour un premier lancement en 2019. - Ces deux actions seront soutenues par l'État à hauteur de 25 millions d'euros. Elles mobiliseront au total un investissement de 54 millions d'euros. Le CNES engagera dès 2014 des contrats de recherche et de développement avec les trois principaux industriels concernés: Thales Alenia Space et Airbus Defence \& Space et Snecma. Les travaux associeront un important réseau d'entreprises de tailles diverses, représentant plus de 1500 emplois directs, situés principalement en Midi-Pyrénées et en Île-de-France. 


\section{ÉQUIPE DU PLAN}

CHEF DE PROJET

Henri Poupart-Lafarge est président d'Alstom Transport.

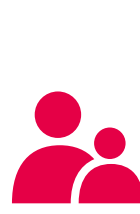

LES INDUSTRIELS

Excent, Xadice, Europe Technologies, Saft, Hutchinson,

Saint Gobain, Leach, Sesaly
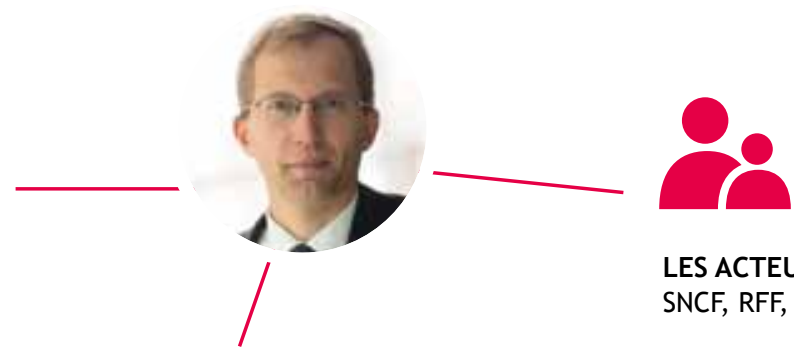

LES ACTEURS PUBLICS

SNCF, RFF, EPSF

\section{LES ECOLES ET ORGANISMES}

DE RECHERCHE

Cetim, FEMTO, Pôle véhicule du futur à Mulhouse,

Université Haute Alsace de Mulhouse,

Université de Technologie Belfort-Montbéliard,

Université de Pau et des Pays de l'Adour,

École Nationale d'Ingénieurs de Tarbes

\section{SYNTHĖSE DES ACTIONS DU PLAN}

Action Calendrier $\begin{aligned} & \text { Acteurs publics } \\ & \text { et levier d'action }\end{aligned} \quad$ Finalités/livrables

Valorisation de l'excellence française en matière de trains à grande vitesse

\begin{tabular}{lccc}
\hline $\begin{array}{l}\text { Mise en œuvre une vitrine du savoir-faire } \\
\text { «TGV France » à l'étranger }\end{array}$ & 2014 & SNCF, État & $\begin{array}{l}\text { Soutien de l'État à la visibilité du savoir-faire de la } \\
\text { Maison France dans la Grande Vitesse }\end{array}$ \\
\hline $\begin{array}{l}\text { Contribution à la définition du modèle } \\
\text { économique du TGV du Futur }\end{array}$ & 2014 & SNCF, RFF, État & $\begin{array}{l}\text { Formalisation des objectifs de l'éco-système } \\
\text { Grande Vitesse en France et à l'export (politiques } \\
\text { d'aménagements du territoire et objectifs économiques) }\end{array}$
\end{tabular}

\section{Prérequis au développement du TGV du Futur}

\begin{tabular}{|c|c|c|c|}
\hline $\begin{array}{l}\text { Contribution à la définition du cahier } \\
\text { des charges du TGV du Futur }\end{array}$ & 2014 & $\begin{array}{l}\text { Filière ferroviaire } \\
\text { de la grande vitesse }\end{array}$ & $\begin{array}{l}\text { Confirmation des besoins futurs en termes de matériel } \\
\text { roulant grande vitesse sur les marchés France et export }\end{array}$ \\
\hline $\begin{array}{l}\text { Consolidation des partenariats avec } \\
\text { les acteurs ferroviaires (PME, ETI, } \\
\text { Clusters, IRT...) }\end{array}$ & 2014 & DGCIS, FIF & $\begin{array}{l}\text { Finalisation des accords de partenariat avec les acteurs } \\
\text { de la filière ferroviaire }\end{array}$ \\
\hline $\begin{array}{l}\text { Préparation des schémas de financement } \\
\text { du plan }\end{array}$ & 2014 & Ademe, CGI & $\begin{array}{l}\text { Soutien de l'État à l'innovation technologique } \\
\text { et/ou en qualité d'investisseur avisé }\end{array}$ \\
\hline \multicolumn{4}{|l|}{ Plan de développement du TGV du Futur } \\
\hline $\begin{array}{l}\text { Développement et sortie usine } \\
\text { d'une première rame de pré-série }\end{array}$ & $\begin{array}{l}36 \text { mois après } \\
\text { signature } 1^{\text {re }} \\
\text { commande }\end{array}$ & SNCF, EPSF & $\begin{array}{l}\text { Rame de présérie disponible pour tests de validation/ } \\
\text { homologation }\end{array}$ \\
\hline $\begin{array}{l}\text { Production et sortie usine d'une première } \\
\text { rame de série }\end{array}$ & $\begin{array}{l}60 \text { mois après } \\
\text { signature } 1^{\text {re }} \\
\text { commande }\end{array}$ & SNCF & Rame de série apte au service commercial \\
\hline
\end{tabular}




\section{TGV DU FUTUR}
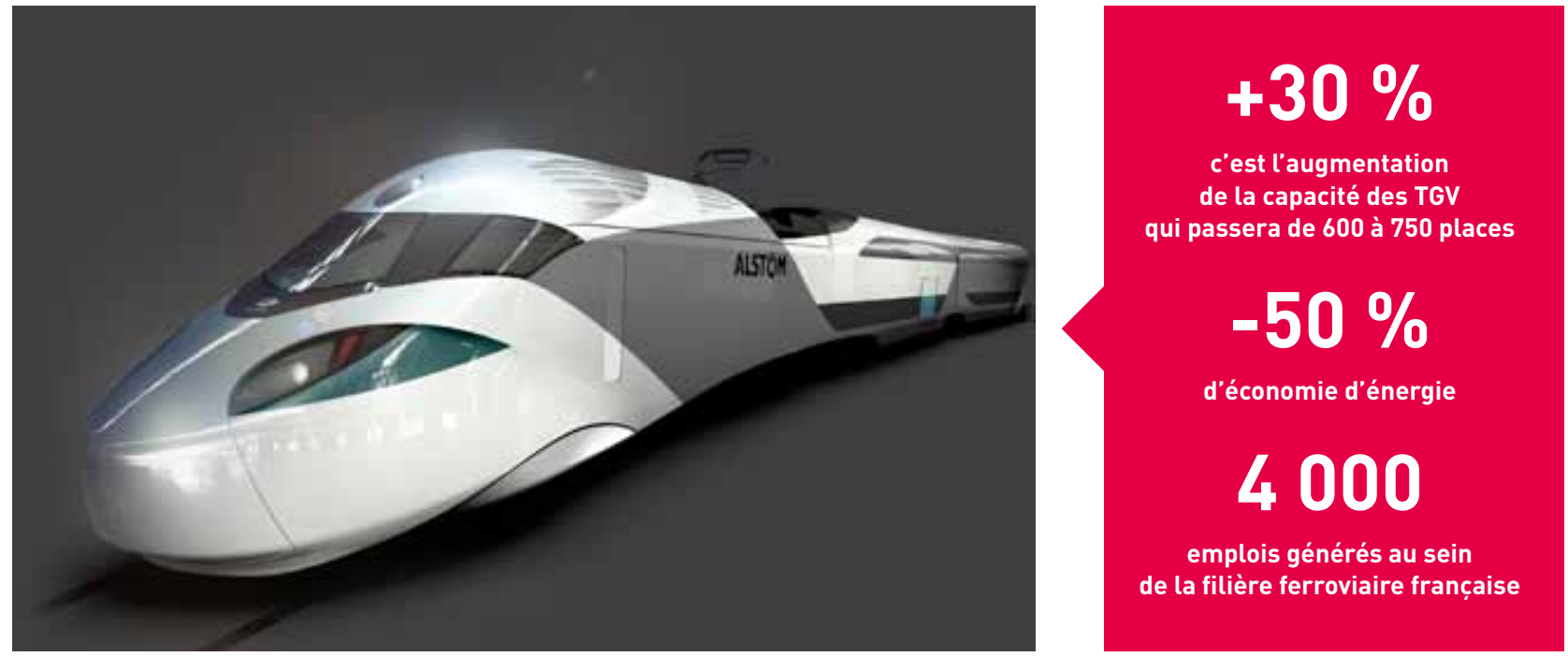

Esquisse du TGV du futur, Alstom

Nous construisons la France de la grande vitesse au service du plus grand nombre. Les performances technologiques de la filière ferroviaire française, pionnière du train à grande vitesse, ont conduit à l'adoption du TGV un peu partout dans le monde. - C'est la raison pour laquelle aujourd'hui la France doit franchir une nouvelle étape de développement technologique à la pointe de l'innovation pour conquérir les nouveaux marchés qui s'ouvrent aux quatre coins du globe. Il lui faut opérer sa révolution ferroviaire et imaginer le TGV du futur pour relever ces défis. - Ce train de demain devra permettre de transporter plus de voyageurs tout en consommant moins d'énergie et en étant plus modulaire. Le plan vise à mobiliser la grande diversité des acteurs industriels et de recherche autour d'Alstom. La collaboration entre la grande entreprise leader mondial reconnu de la grande vitesse et un tissu performant d'opérateurs ferroviaires et de partenaires industriels, doit permettre de créer près de 4000 emplois au sein de la filière. L'État accompagne ces efforts, au travers notamment du programme des investissements d'avenir et a lancé en janvier 2014 un nouvel appel à projets doté de 120 millions d'euros. - Compte tenu du caractère fortement diffusant des innovations prévues, les résultats du plan TGV du Futur pourront s'étendre à d'autres matériels roulants comme les trains régionaux, les métros ou les tramways. - Le plan a vocation à maintenir la France parmi les principaux acteurs mondiaux dans le domaine ferroviaire. 


\section{ÉQUIPE DU PLAN}

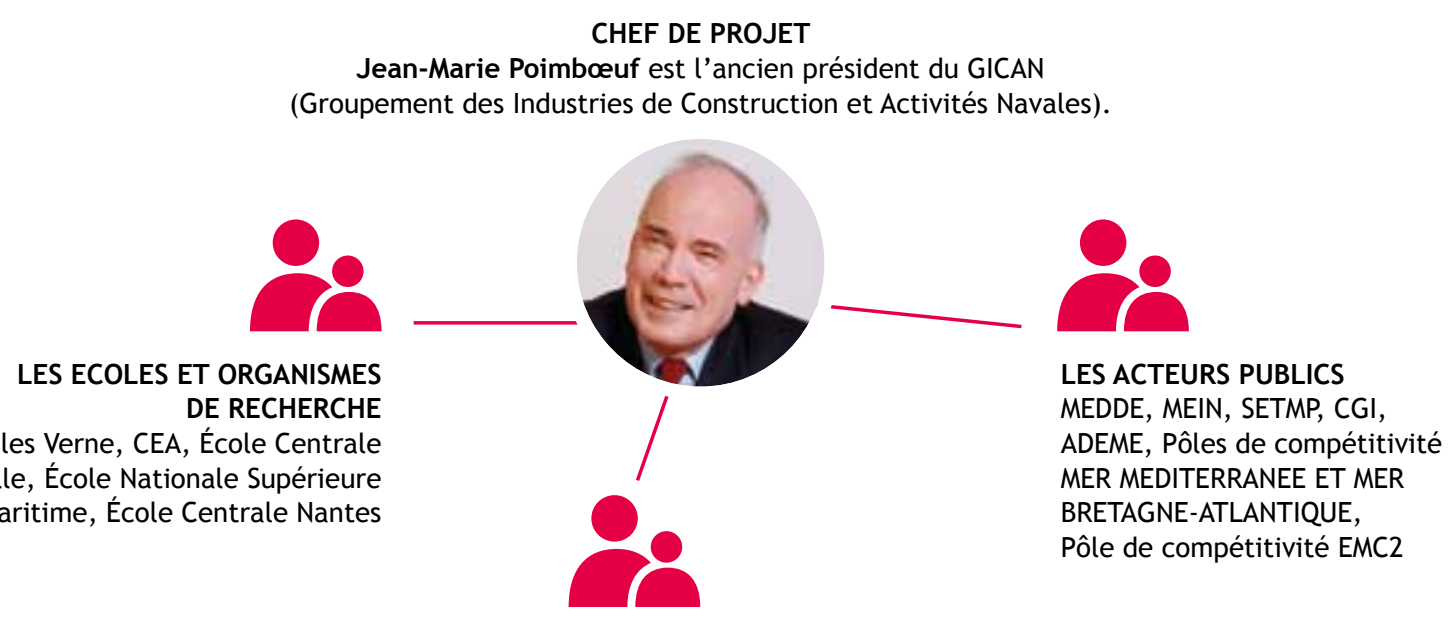

LES INDUSTRIELS ET ASSOCIATIONS PROFESSIONNELLES AIR LIQUIDE, BUREAU VERITAS, CRYOGENIC CONTAINMENT

(Europe Technologies), DAMEN SHIPREPAIR, DCNS, FMC

TECHNOLOGIES, GTT, LDA, MAXSEA, STX France, SAGEM, Association française du gaz, Armateurs de France, BP2S, ENSM, Union des ports de France, Elios Lvs

\section{SYNTHÈSE DES ACTIONS DU PLAN}

\begin{tabular}{|c|c|c|c|}
\hline Action & Pilote & Calendrier & Livrables \\
\hline \multicolumn{4}{|c|}{ Relever le défi énergétique: GNL, énergie électrique et propulsion vélique } \\
\hline \multicolumn{4}{|l|}{$\begin{array}{l}\text { Développement conjoint d'une offre industrielle } \\
\text { française et de l'utilisation du GNL comme carburant } \\
\text { marin : }\end{array}$} \\
\hline $\begin{array}{l}\text { Mise en place un démonstrateur de soute GNL, } \\
\text { centre R\&D et de formation, et vitrine commerciale }\end{array}$ & $\begin{array}{l}\text { Cryogenic } \\
\text { Containment }\end{array}$ & 2016 & Démonstrateur soute GNL \\
\hline $\begin{array}{l}\text { Accompagnement des armateurs dans leurs projets } \\
\text { d'investissement dans des ferries propres }\end{array}$ & ADEME & 2014 & Flottes rénovées \\
\hline $\begin{array}{l}\text { Accompagnement du déploiement des infrastructures } \\
\text { portuaires dédiées à l'utilisation du GNL }\end{array}$ & $\begin{array}{l}\text { Mission de } \\
\text { coordination GNL }\end{array}$ & & Stations d'approvisionnement en GNL \\
\hline $\begin{array}{l}\text { Développement de navires intégrant une électrification } \\
\text { optimale, grâce à la filière Hydrogène pour la Pêche } \\
\text { polyvalente (FILHyPyNE ) }\end{array}$ & DCNS- STX & 2018 & PAC pour navires \\
\hline \multicolumn{4}{|l|}{$\begin{array}{l}\text { Utilisation de la force du vent par le vélique : } \\
\text { Propulsion auxiliaire des navires }\end{array}$} \\
\hline $\begin{array}{l}\text { Développement d’un prototype paquebot à propulsion } \\
\text { hybride vélique-électrique de nouvelle génération }\end{array}$ & STX & 2016 & $\begin{array}{l}\text { Prototype paquebot hybride voile/électrique } \\
\text { (Silenseas) }\end{array}$ \\
\hline \multicolumn{4}{|l|}{ Développer une passerelle intelligente } \\
\hline Passerelles maritimes intelligentes (projet PAMI) & SAGEM & 2015 à 2018 & $\begin{array}{l}\text { Passerelles intégrées complètes et équipements } \\
\text { (ECDIS, autopilote, BAMS, radar FMCW, système } \\
\text { de communication IP, tourelle EO/IR) }\end{array}$ \\
\hline \multicolumn{4}{|l|}{ Tendre vers un navire le plus éco-efficient } \\
\hline $\begin{array}{l}\text { Réunion des acteurs industriels autour d'un projet } \\
\text { de développement, d'industrialisation, } \\
\text { de maritimisation d'un système de traitement } \\
\text { des rejets }\end{array}$ & DCNS/STX & 2014 à 2020 & Système de traitement \\
\hline Réduction des consommations de carburants & Mauric & 2015 à 2018 & Rame de série apte au service commercial \\
\hline \multicolumn{4}{|l|}{ Renforcer les fonds propres et les capacités export } \\
\hline $\begin{array}{l}\text { Renforcement des fonds propres et des capacités } \\
\text { export des PME et ETI de la filière navale française }\end{array}$ & GICAN & A partir 2014 & \\
\hline
\end{tabular}




\section{NAVIRES ÉCOLOGIQUES}
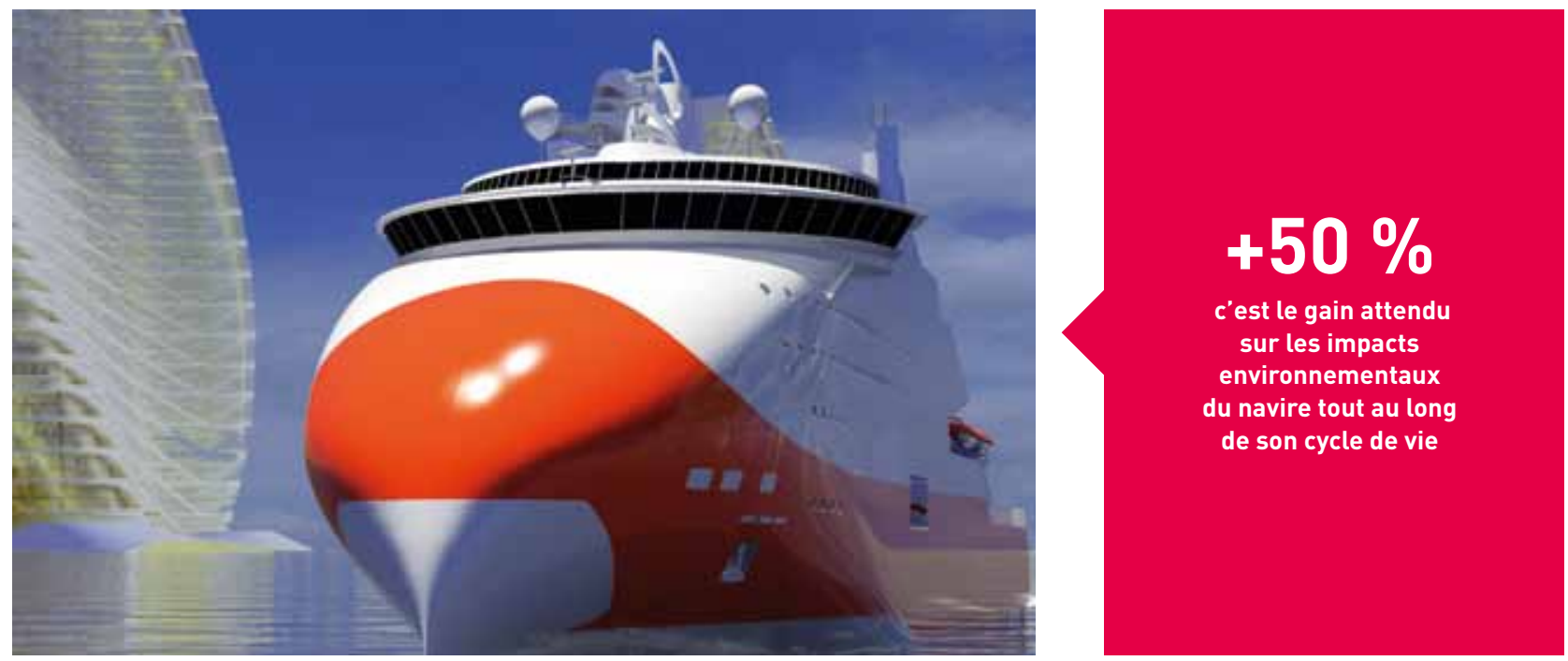

Projet de navire écologique, STX France

Nous construisons la France des navires sobres et écologiques. Dans un environnement mondial très fortement concurrentiel, la filière navale française a l'ambition d'asseoir sa compétitivité sur l'excellence de ses solutions technologiques, capables de répondre aussi bien aux défis environnementaux qu'aux exigences économiques et de sécurité. Elle veut profiter des solides perspectives de développement de l'industrie navale, dans un monde où près de $90 \%$ des échanges de marchandises transitent par les mers et océans, et où le développement considérable des énergies marines renouvelables et du trafic fluvial devrait encore s'accélérer d'ici à 2020. - En grande partie structurée autour des têtes de filières STX et DCNS, de chantiers performants et d'un tissu de coopérants et d'équipementiers dynamiques, l'industrie navale française dispose de tous les métiers nécessaires au développement, à la construction, à la réparation et à la transformation de navires et équipements, incluant les technologies de l'offshore. - Le plan a pour objectif de concentrer ses actions d'abord sur la protection de l'environnement avec en jeu un gain de $50 \%$ sur l'ensemble des impacts environnementaux du navire tout au long de son cycle de vie, ensuite sur l'amélioration de la sécurité et la sûreté des passagers, équipages et cargaisons et enfin sur l'intelligence et l'intégration des navires et équipements pour réduire leurs coûts de fonctionnement. 


\section{ÉQUIPE DU PLAN}

CHEF DE PROJET

Yves Dubief est Président de l'Union des industries Textiles (UIT) et Président de Tenthorey.

LES INDUSTRIELS

Thuasne, Safilin, Schappe,

Damartex, Texinov,

Cityzen Sciences,

Sherpa Finance, Uric-unimaille

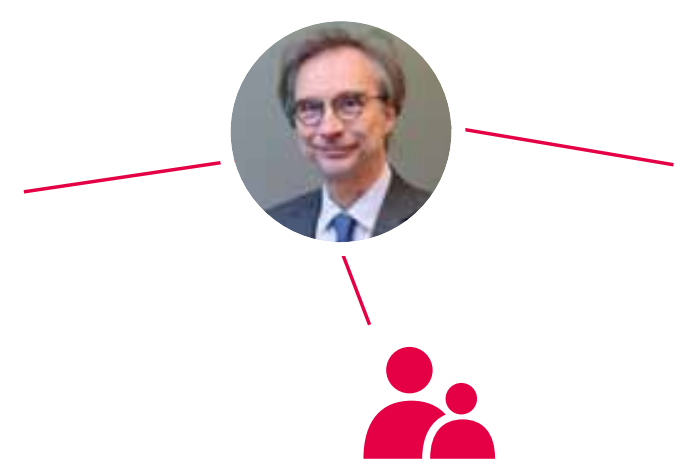

LES ACTEURS PUBLICS

Bpifrance, CGI, MENESR, MAAF

\section{SYNTHÈSE DES ACTIONS DU PLAN}

\begin{tabular}{|c|c|c|c|c|}
\hline Action & Pilote & Calendrier & Acteurs privés concernés & Acteurs publics concernés \\
\hline $\begin{array}{l}\text { Lancement d'un appel à } \\
\text { projets sur les thématiques } \\
\text { du plan pour faire } \\
\text { émerger et accompagner } \\
\text { financièrement les projets } \\
\text { des industriels } \\
\text { du textile et de } \\
\text { l'habillement }\end{array}$ & & $\begin{array}{l}\text { Second semestre } \\
2014\end{array}$ & $\begin{array}{l}\text { UIT, IFTH, CETI, Pôles de } \\
\text { compétitivité (Up-Tex, } \\
\text { Techtera, Fibres), Instituts } \\
\text { Carnot, Association des } \\
\text { structures de recherche sous } \\
\text { contrat (ASRC), Union des } \\
\text { constructeurs de matériel } \\
\text { textile de France (UCMTF) }\end{array}$ & CGI, Bpifrance, Direccte, MENESR, MAAF \\
\hline $\begin{array}{l}\text { Conception, développement } \\
\text { et commercialisation de } \\
\text { cinq produits grand public } \\
\text { en textiles connectés, qui } \\
\text { prendront valeur d'exemple } \\
\text { auprès } \\
\text { des industriels du textile } \\
\text { (projet « Connectitude») }\end{array}$ & $\begin{array}{l}\text { Réseau pour } \\
\text { l'innovation } \\
\text { immatérielle } \\
\text { dans l'industrie } \\
\text { (R3iLab) }\end{array}$ & $\begin{array}{l}\text { Du second } \\
\text { semestre } 2014 \\
\text { à la fin } 2015\end{array}$ & $\begin{array}{l}\text { IFTH, CETI, Pôles de } \\
\text { compétitivité (Up-Tex, } \\
\text { Techtera, Fibres), UIT, Prix } \\
\text { Théophile Legrand, ENSAIT, } \\
\text { L'Usine du futur }\end{array}$ & MEIN, Bpifrance \\
\hline $\begin{array}{l}\text { Élaboration et diffusion des } \\
\text { scenarii les plus probables } \\
\text { d'évolution des marchés } \\
\text { et des conditions de } \\
\text { compétitivité de la filière } \\
\text { textile afin d'éclairer } \\
\text { les choix stratégiques } \\
\text { des industriels (projet } \\
\text { « Futurs immédiats") }\end{array}$ & $\begin{array}{l}\text { Réseau pour } \\
\text { l'innovation } \\
\text { immatérielle } \\
\text { dans l'industrie } \\
\text { (R3iLab) }\end{array}$ & $\begin{array}{l}\text { Du second } \\
\text { semestre } 2014 \\
\text { à la fin } 2015\end{array}$ & $\begin{array}{l}\text { IFTH, CETI, Pôles de } \\
\text { compétitivité (Up-Tex, } \\
\text { Techtera, Fibres), ENSAIT, } \\
\text { L'Usine du futur, Cap Digital, } \\
\text { Établissements de formation } \\
\text { au design industriel (ENSCI ; } \\
\text { ESAD), Agence Nelly Rodi }\end{array}$ & MEIN \\
\hline $\begin{array}{l}\text { Identification des besoins } \\
\text { en textiles innovants des } \\
\text { personnels publics civils } \\
\text { et militaires (habillement, } \\
\text { matériels divers, } \\
\text { équipements connectés...) }\end{array}$ & MEIN et UIT & $\begin{array}{l}\text { Second semestre } \\
2014\end{array}$ & $\begin{array}{l}\text { Tous industriels textiles; IFTH ; } \\
\text { R3iLab }\end{array}$ & $\begin{array}{l}\text { MEIN ; Médiation des marchés publics ; MINDEF ; } \\
\text { Ministère de l'Intérieur ; EDF ; } \\
\text { GDF-Suez }\end{array}$ \\
\hline
\end{tabular}




\section{TEXTILES TECHNIQUES ET INTELLIGENTS}
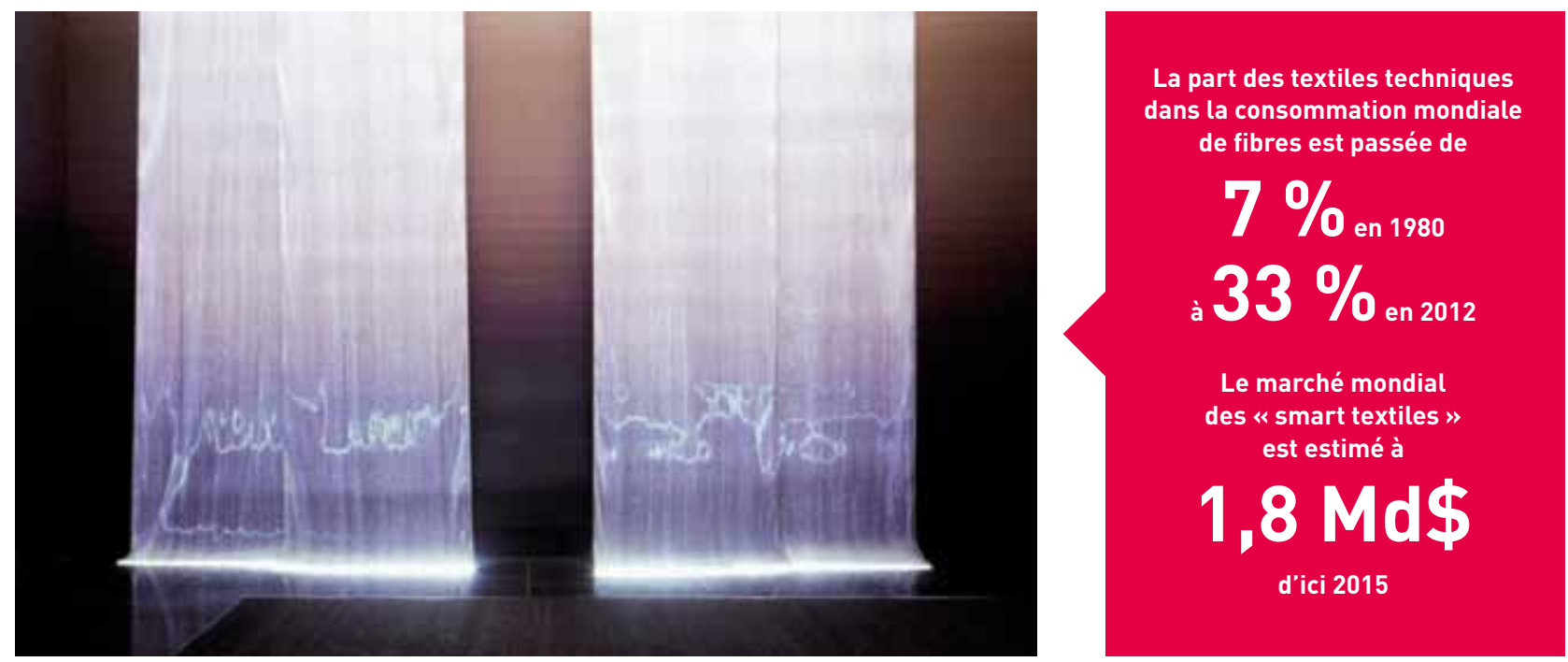

Rideaux lumineux, Brochier Technologies

- Nous construisons la France du textile innovant. Symbole de la première révolution industrielle, l'industrie textile est en constante évolution et se présente encore aujourd'hui comme un secteur d'avenir, fortement innovant. Le textile de demain n'aura pas les mêmes fonctions que celui d'hier. Plus technologique et respectueux de l'environnement, il pourra offrir de nouveaux services dans tous les domaines. Il sera curatif, communicant, capable de capter l'énergie solaire ou de donner des informations corporelles. L'arrivée du textile technique entraîne un courant d'innovation qui transforme l'ensemble des métiers de la filière.

- La France a les moyens d'être leader dans les projets industriels de demain en matière de textiles innovants. Elle dispose d'un réseau d'entreprises performantes, épaulées par des laboratoires de recherche de pointe, des écoles d'ingénieurs reconnues, des structures comme l'Institut français du textile et de l'habillement (IFTH) ou le Centre européen des textiles innovants (CETI) et des pôles de compétitivité dynamiques (Uptex, Techtera, Fibres). Qualification des personnels, maîtrise des matériaux, incorporation des avancées technologiques sont autant d'autres avantages essentiels qui la positionnent comme un acteur clé du futur. — L'État s'engage aux côtés des régions textiles et de l'écosystème scientifique et industriel pour faire émerger de nouveaux produits en facilitant leur financement et en leur offrant de nouveaux débouchés. Ainsi, le plan dédié aux textiles innovants vise tout d'abord à réussir la transition écologique, en maîtrisant et diffusant l'usage de fibres textiles biosourcées telles que le chanvre et de fibres issues du recyclage. Ensuite, il exploitera les opportunités offertes par les révolutions numériques et les nanotechnologies en intégrant plus d'intelligence aux textiles de demain. Enfin, il développera l'usine textile du futur, grâce aux nouvelles technologies d'ennoblissement et d'assemblage et à une utilisation optimale des nouveaux outils digitaux. 


\section{ÉQUIPE DU PLAN}

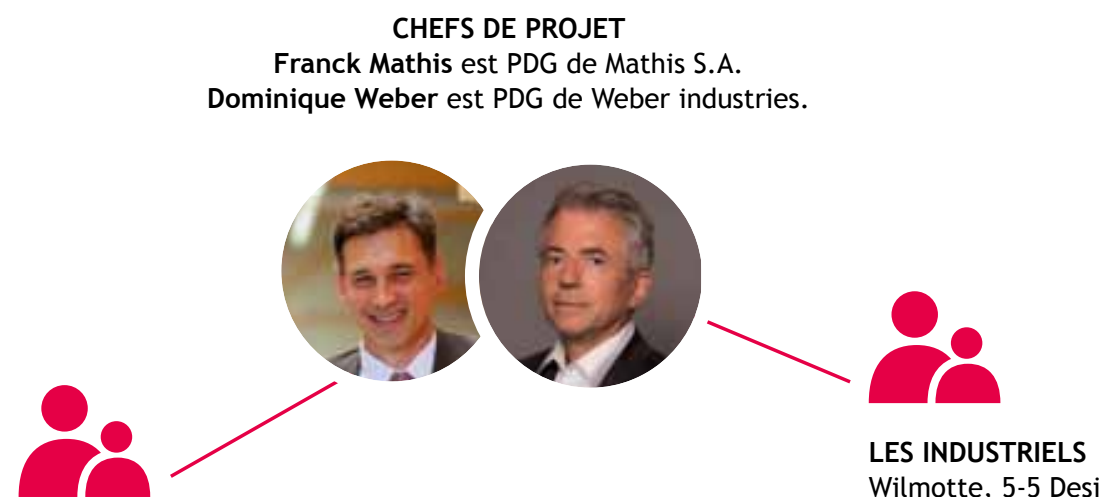

Wilmotte, 5-5 Design Studio, Setec, Egis, Apave, Terrell group, Nexity,

LES ACTEURS PUBLICS Icade, Union Habitat, Toit Vosgien, MEIN, MLET, MAAF, MI, CGI, Bpifrance, FCBA, Eiffage, Arbonis, Woodeum, SIAT Braun, Mathis, Salm, Majencia, CSTB, CDC Lapeyre, MGD, Weber, FIBC, UNIFA

\section{SYNTHĖSE DES ACTIONS DU PLAN}

\begin{tabular}{|c|c|c|c|}
\hline Action & Pilote & Calendrier & Finalités/livrables \\
\hline $\begin{array}{l}\text { Création d'une structure commune } \\
\text { de portage et de gouvernance du plan }\end{array}$ & ADIVBois & Dès le second semestre 2014 & $\begin{array}{l}\text { Implication des industriels de la construction } \\
\text { et de l'ameublement bois et de son écosystème } \\
\text { dans la mise en œuvre du plan }\end{array}$ \\
\hline $\begin{array}{l}\text { Simplification réglementaire et normative } \\
\text { pour lever les freins à l'offre en } \\
\text { immeubles de grande hauteur en bois }\end{array}$ & $\begin{array}{l}\text { Commission } \\
\text { technique et } \\
\text { réglementaire } \\
\text { ad hoc }\end{array}$ & $\begin{array}{l}\text { Du second semestre } 2014 \\
\text { à mi } 2015\end{array}$ & Mesures de simplification pour les immeubles en bois \\
\hline \multirow[t]{2}{*}{$\begin{array}{l}\text { Projets pilotes immeubles de grande } \\
\text { hauteur }(\mathrm{IGH}) \text { en bois }\end{array}$} & ADIVBois & Dès le second semestre 2014 & $\begin{array}{l}\text { État des marchés ciblés en France et à l'étranger } \\
\text { pour les immeubles en bois et les produits issus de bois } \\
\text { français, benchmarking des solutions techniques } \\
\text { et dispositions réglementaires pour les IGH bois } \\
\text { dans le monde }\end{array}$ \\
\hline & & & Définition et test des solutions techniques \\
\hline $\begin{array}{l}\text { Amorçage du marché par la commande } \\
\text { publique }\end{array}$ & $\begin{array}{l}\text { État et } \\
\text { Collectivités } \\
\text { locales }\end{array}$ & $\begin{array}{l}\text { Dès le second semestre } 2014 \\
\text { à mi } 2015 \text {, puis à partir de } \\
\text { mi } 2015 \text { pour les actions post } \\
\text { concours }\end{array}$ & $\begin{array}{l}\text { Concours national organisé par l'État, appel à } \\
\text { candidatures pour sélectionner des collectivités } \\
\text { qui s'engageraient à lancer deux ou trois appels } \\
\text { à projets par an }\end{array}$ \\
\hline Structuration de l'offre & Industriels & Dès le second semestre 2014 & $\begin{array}{l}\text { Disposer d'une offre compétitive française sur le marché } \\
\text { international de produits d'immeuble en bois (structure } \\
\text { et ameublement) de plus de } 15 \text { niveaux avec des } \\
\text { démonstrateurs sur le territoire français }\end{array}$ \\
\hline
\end{tabular}




\section{INDUSTRIES DU BOIS}
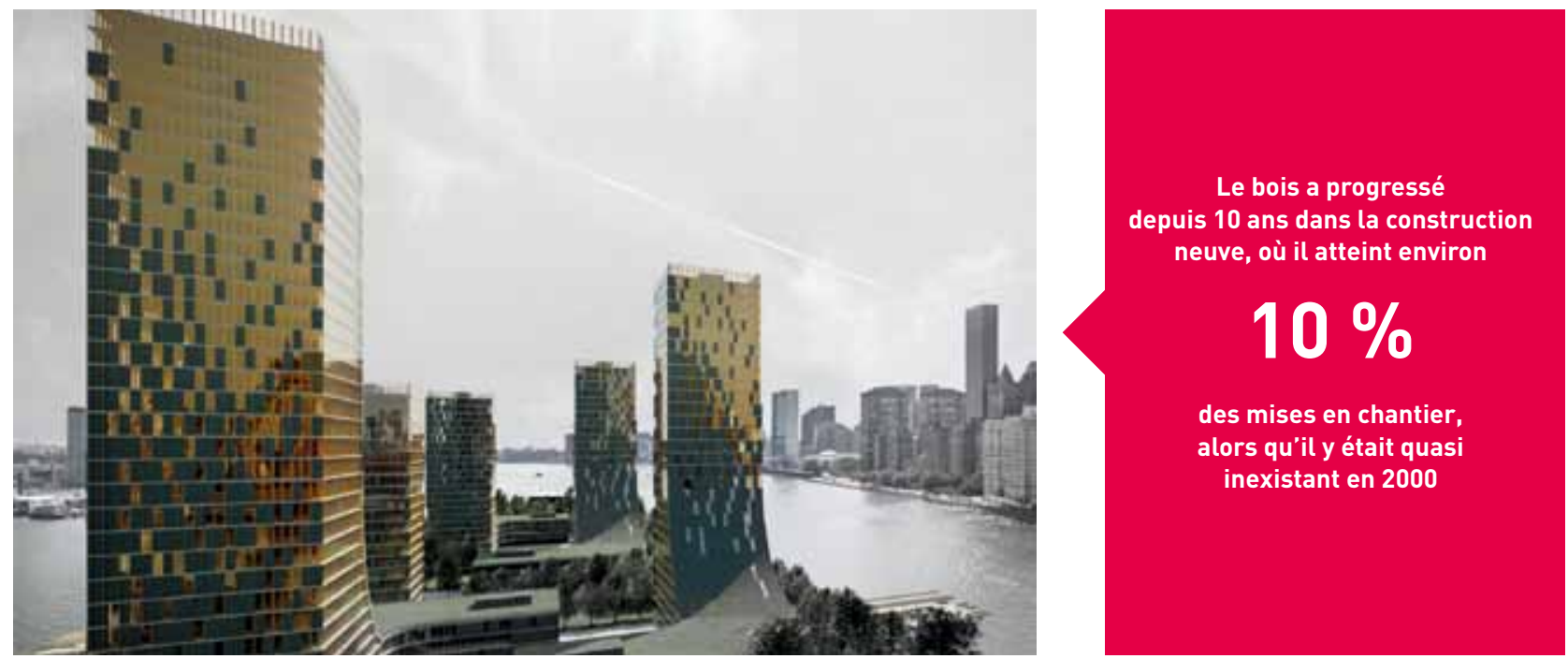

Immeuble Michael Green en ossature bois

Nous construisons la France de l'or vert où la forêt est une ressource d'avenir. Les propriétés techniques, économiques et environnementales du bois en font un matériau stratégique aux usages multiples : construction, ameublement, chimie, papier, biomasse... Il est désormais possible de construire de grands immeubles en ossature bois et de valoriser les sous-produits de l'industrie. Les innovations techniques offrent de fortes perspectives de croissance économique et de création d'emplois dans les industries du bois. - Troisième surface forestière d'Europe, la France fait face à un paradoxe: nous exportons notre bois fraîchement sorti de nos forêts et importons les produits finis à plus forte valeur ajoutée. Résultat : un poste déficitaire de plus de 6 milliards d'euros par an dans notre balance commerciale. - Le plan « Industries du bois » va corriger ce déséquilibre en réimplantant sur le territoire national les activités de transformation et en accélérant l'industrialisation de l'offre. Le bois de France, transformé en France, fournira ainsi matériaux de construction, biens de consommation et énergie, tout en représentant un potentiel pouvant aller jusqu'à 60000 emplois nouveaux notamment dans les zones rurales. Ce plan permettra de faire des forêts de France la source renouvelable d'une industrie moderne, innovante et responsable. - Il s'agit de mobiliser les industriels de la construction et de l'ameublement autour d'un objectif commun : parvenir à construire en France un immeuble de 30 étages en 2030. Le plan se fixe comme objectif intermédiaire de bâtir à l'horizon 2017, de 5 à 10 vrais immeubles urbains de moyenne hauteur (7 à 15 étages) dans des territoires. — La commande publique et des mesures de simplification réglementaire seront actionnées pour amorcer le marché. Ces premiers débouchés permettront à l'offre française de se constituer pour ensuite se lancer à l'international. 


\section{ÉQUIPE DU PLAN}

CHEF DE PROJET

Antoine Frérot est PDG de Veolia.

\section{LES INDUSTRIELS ET FÉDÉRATIONS}

PROFESSIONNELLES

Veolia, Suez Environnement, Séché

Environnement, PAPREC, Pellenc ST,

Siléane, FNADE, FEDEREC

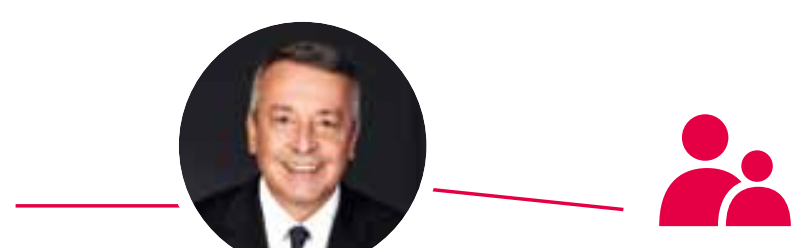

LES ACTEURS PUBLICS

MEIN, MEDDE, MENESR

et Régions Auvergne,

Basse-Normandie, Bourgogne, Champagne-Ardenne, Limousin et Nord-Pas-de-Calais

\section{SYNTHĖSE DES ACTIONS DU PLAN}

\begin{tabular}{|c|c|c|c|}
\hline Action & Calendrier & $\begin{array}{l}\text { Acteurs privés et publics, } \\
\text { leviers d'actions }\end{array}$ & $\begin{array}{l}\text { Finalités/livrables acteurs } \\
\text { publics }\end{array}$ \\
\hline \multicolumn{4}{|c|}{ Réduire les mises en décharge et développer des infrastructures de tri et de valorisation } \\
\hline $\begin{array}{l}\text { Réduction des mises en stockage, extension ou création } \\
\text { de centres de tri et de recyclage, visibilité sur la TGAP, } \\
\text { stabilité de la fiscalité globale des déchets, garanties } \\
\text { contre les risques d'impayés }\end{array}$ & $\begin{array}{l}\text { Dès } 2014 \\
\text { et horizon } 2020\end{array}$ & $\begin{array}{l}\text { Appui du CGI, } \\
\text { arrêtés préfectoraux, } \\
\text { loi de finances }\end{array}$ & $\begin{array}{l}50 \text { projets identifiés à examiner à l'aune } \\
\text { du maillage territorial }\end{array}$ \\
\hline \multicolumn{4}{|l|}{ Mieux valoriser énergétiquement les déchets } \\
\hline $\begin{array}{l}\text { Meilleur valorisation du potentiel énergétique des usines } \\
\text { d'incinération }\end{array}$ & $2014 / 2015$ & & $\begin{array}{l}\text { Expérimentation sur } 3 \text { sites, } \\
\text { puis éventuelle extension }\end{array}$ \\
\hline $\begin{array}{l}\text { Développement des CSR en clarifiant leur statut juridique, } \\
\text { en les rendant éligibles aux aides pour les ENR, en créant } \\
\text { des centres de production et combustion des CSR }\end{array}$ & $2014 / 2015$ & Ministères concernés & $\begin{array}{l}31 \text { projets associant centres de tri } \\
\text { et CSR }\end{array}$ \\
\hline \multicolumn{4}{|l|}{ Mieux valoriser les plastiques et soutenir la R\&D } \\
\hline Recours aux leviers législatif et réglementaire & 2015 & $\begin{array}{l}\text { Commission consultative } \\
\text { sur le statut de déchet }\end{array}$ & $\begin{array}{l}\text { Sortie des plastiques recyclés } \\
\text { du statut « déchets» }\end{array}$ \\
\hline Encouragement de la demande de plastiques recyclés & 2015 & $\begin{array}{l}\text { Ministères concernés, recours } \\
\text { notamment à une TVA à taux } \\
\text { réduit et à la commande } \\
\text { publique }\end{array}$ & $\begin{array}{l}\text { Augmentation de l'utilisation } \\
\text { des plastiques recyclés }\end{array}$ \\
\hline $\begin{array}{l}\text { Création ou extension de centres de tri et de valorisation } \\
\text { des déchets }\end{array}$ & 2014 & Appui du CGI & 18 projets identifiés \\
\hline \multicolumn{4}{|l|}{ Mieux valoriser les déchets du BTP } \\
\hline $\begin{array}{l}\text { Lutte contre les décharges illégales et création de centres } \\
\text { de tri et de valorisation des déchets }\end{array}$ & $2014 / 2015$ & $\begin{array}{l}\text { État, Appui du CGI (centres } \\
\text { de tri) }\end{array}$ & \\
\hline Établissement de normes en matière de recyclats & 2015 & $\begin{array}{l}\text { Fédérations professionnelles, } \\
\text { Ministères concernés }\end{array}$ & \\
\hline Recours aux leviers législatif et réglementaire & 2015 & $\begin{array}{l}\text { Commission consultative } \\
\text { sur le statut de déchet }\end{array}$ & $\begin{array}{l}\text { Sortie des matières recyclées issues } \\
\text { des déchets du BTP du statut « déchets " }\end{array}$ \\
\hline \multicolumn{4}{|c|}{ Mieux valoriser les fibres de carbone et soutenir la R\&D avec l'IRT Jules Verne } \\
\hline Amélioration de l'accès aux gisements & 2015 & Ministères concernés & $\begin{array}{l}\text { Attirer les avions en fin de vie en France } \\
\text { pour alimenter le secteur recyclage }\end{array}$ \\
\hline $\begin{array}{l}\text { Soutien à la demande de fibres recyclées } \\
\text { (notamment par une TVA à taux réduit) }\end{array}$ & 2015 & Ministères concernés & \\
\hline Recours aux leviers législatif et réglementaire & 2015 & $\begin{array}{l}\text { Commission consultative } \\
\text { sur le statut de déchet }\end{array}$ & $\begin{array}{l}\text { Sortie des fibres recyclées du statut } \\
\text { déchet }\end{array}$ \\
\hline Création des centres de tri et de valorisation & 2015 & Appui du CGI & 2 projets identifiés \\
\hline \multicolumn{4}{|l|}{ Mieux valoriser les cartes électroniques } \\
\hline $\begin{array}{l}\text { Massification des gisements, notamment par des dispositifs } \\
\text { de type consigne }\end{array}$ & 2015 & $\begin{array}{l}\text { Fédérations professionnelles, } \\
\text { Ministères concernés }\end{array}$ & \\
\hline $\begin{array}{l}\text { Création de centres de tri et valorisation, reposant } \\
\text { sur de nouvelles technologies d'extraction des métaux }\end{array}$ & $2014 / 2015$ & Appui du CGI & 6 projets identifiés \\
\hline
\end{tabular}




\section{RECYCLAGE ET MATÉRIAUX VERTS}
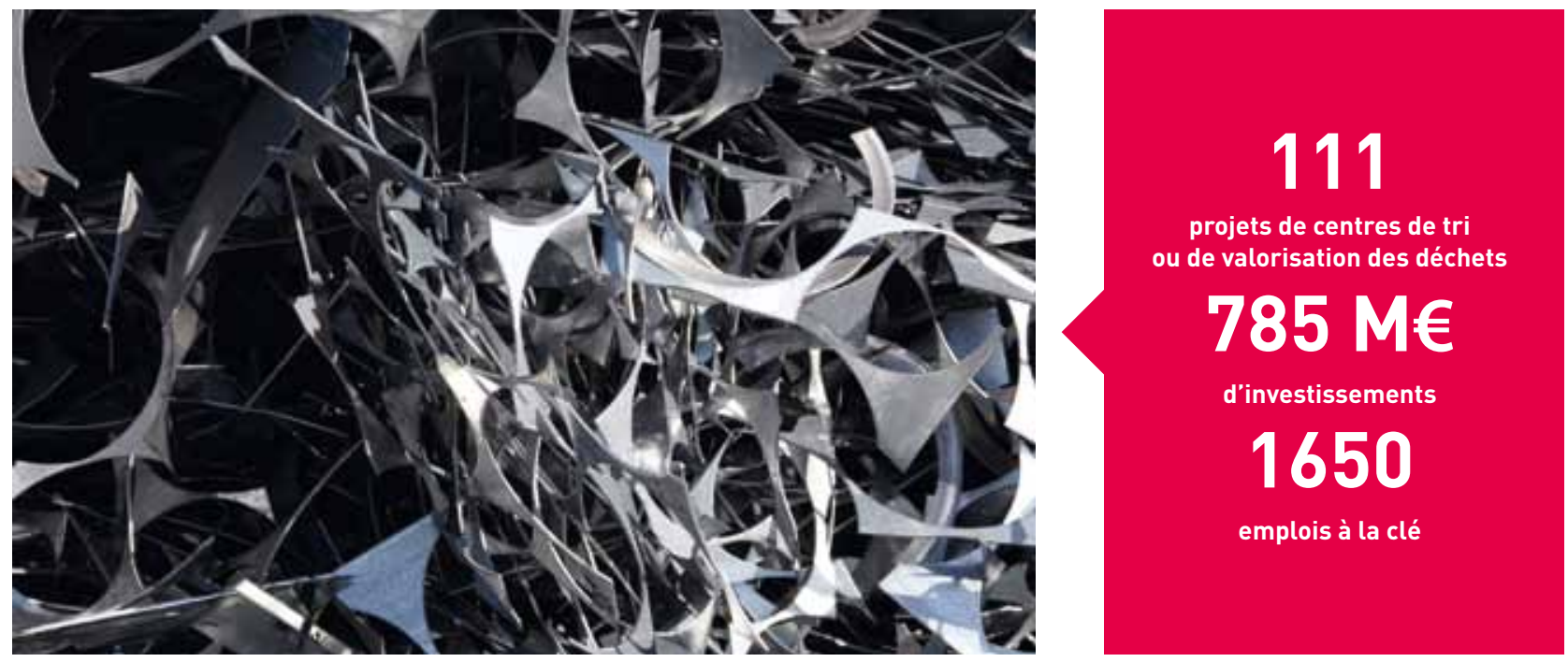

Recyclage métal

- Nous construisons la France industrielle éco-responsable.

- Recycler est une priorité en termes d'environnement : une tonne de papier recyclé permet d'économiser 830 litres de pétrole. Recycler est une priorité en termes d'emplois : recycler les déchets industriels crée 6 fois plus d'emplois que de les enfouir. Recycler est une priorité en termes de compétitivité : dans l'industrie papetière, des entreprises viennent s'installer en France pour bénéficier de la matière première recyclée existante sur notre territoire. \ Les industriels des déchets et du recyclage soutiennent une réduction importante, par voie réglementaire, des mises en décharge (-30\% de déchets en 2020 , $-50 \%$ en 2025$)$ et se mobilisent pour 111 projets de centres de tri ou de valorisation remontés dans le cadre du plan, représentant 785 M€ d'investissements et 1650 emplois directs. - Avec eux, le plan vise également à renforcer la valorisation énergétique des déchets, en développant notamment les Combustibles Solides de Récupération (CSR), source importante d'énergie renouvelable. - Pour que ces projets d'investissement voient le jour, l'environnement économique, législatif et fiscal du secteur devra être stable, pour permettre aux industriels d'avoir la visibilité nécessaire pour engager des investissements amortissables sur plusieurs années. - Le CGI est également sollicité pour appuyer les projets d'investissement, dans le cadre de l'Appel à manifestation d'intérêt « économie circulaire ». — Les industriels se mobilisent enfin sur quatre filières spécifiques de recyclage, choisies en raison des volumes de déchets traités (la filière plastique et les déchets du BTP), ou du caractère innovant des matières concernées (les fibres de carbone et les cartes électroniques). - Pour chacune de ces filières, le plan industriel propose un plan d'action spécifique : soutien à la R\&D, amélioration de l'accès au gisement, développement de la demande de matières premières recyclées, création d'infrastructures industrielles, etc. 


\title{
ÉQUIPE DU PLAN
}

\section{CHEFS DE PROJET}

Jacques Pestre est directeur général adjoint de Point $P$. Marcel Torrents est président du directoire de Delta Dore.

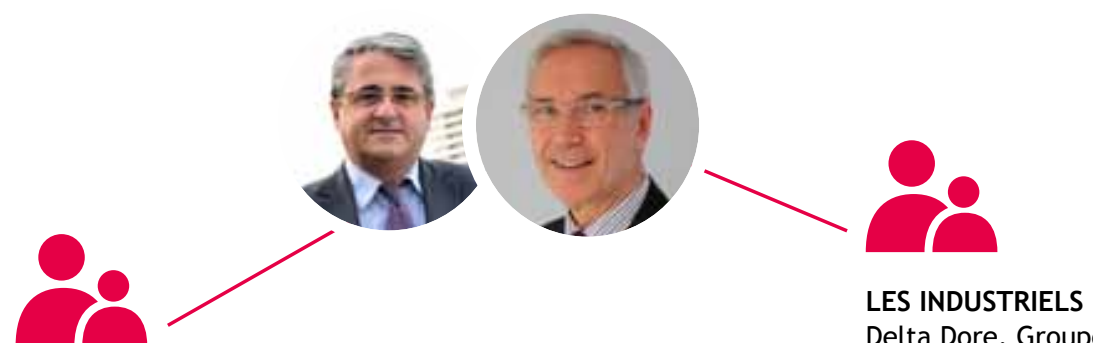

Delta Dore, Groupe Point P, Groupe Atlantic, Schneider Electric, Aldes, Velux France, LES ACTEURS PUBLICS MEIN, CGI, Bpifrance, DHUP, MENESR

Terreal, Capeb, FFB, Qualibat, Plan Bâtiment durable

\section{SYNTHÈSE DES ACTIONS DU PLAN}

Action

Calendrier

Engagement/objectif

Un engagement ferme des industriels et du négoce

Création du «Cercle des Industriels et des Négoces

de la rénovation» (CINR)

\author{
2014
} de la filière pour la performance de la rénovation au profit du consommateur

d'ici 2015

Déclinaison en cercles régionaux des décideurs de la rénovation (CERDR)

Promouvoir l'efficacité énergétique dans tous les travaux significatifs de rénovation

Campagne de communication de la filière industrielle et du négoce et développement d'offres produits packagées permettant d'integrer facilement l'efficacite énergetique

d'ici 2015 en coordination avec Inciter à l'intégration de l'efficacité énergétique dans la rénovation

Le RGE pour structurer la montée en compétence des professionnels de la rénovation énergétique

Mobilisation générale de l'industrie, du négoce, des artisans Second semestre 2014 et entreprises pour le RGE

Second semestre 2014 à 2017

Accompagnement des formations et mise en place d'outils de sensibilisation de proximité par les professionnels du négoce et création du RGE Négoce

Labellisation de 18000 professionnels au 1er juillet 2014 et de 30000 un an plus tard

Accompagnement de la montée en compétence des

Second semestre 2014

professionnels et renforcement du signe de qualité du RGE

Mise en place d'outils de formation pratiques, didactiques, adaptés et continus, en fonction des exigences du RGE.

exigences du RGE.
Convergence des pratiques des organismes certificateurs.

Montée en puissance des outils de regroupement d'entreprises Second semestre 2014

Émergence d'offres globales par des groupements d'entreprises

Mobilisation du fonds de compensation des risques

d'assurance-construction, pour soutenir les actions de

Second semestre 2014

Développer des outils innovants permettant de palier à la sinistralité spécifique à la rénovation prévention de la sinistralité en rénovation énergétique énergétique

\begin{tabular}{lll}
\hline Préparer un leadership mondial dans la rénovation 3.0 & & \\
\hline Emergence d'un écosystème de la rénovation 3.0 & 2014 & $\begin{array}{l}\text { Mise en place d'un feuille de route « rénovation } \\
3.0 \text { ", partagée entre acteurs du numérique et du } \\
\text { bâtiment }\end{array}$ \\
\hline Soutien à l'interopérabilité domotique & $2014-2015$, export en 2016 & $\begin{array}{l}\text { Mise au point d'un protocole d'interopérabilité } \\
\text { permettant la liaison entre les différents } \\
\text { équipements }\end{array}$
\end{tabular}




\section{RÉNOVATION THERMIQUE DES BÂTIMENTS}
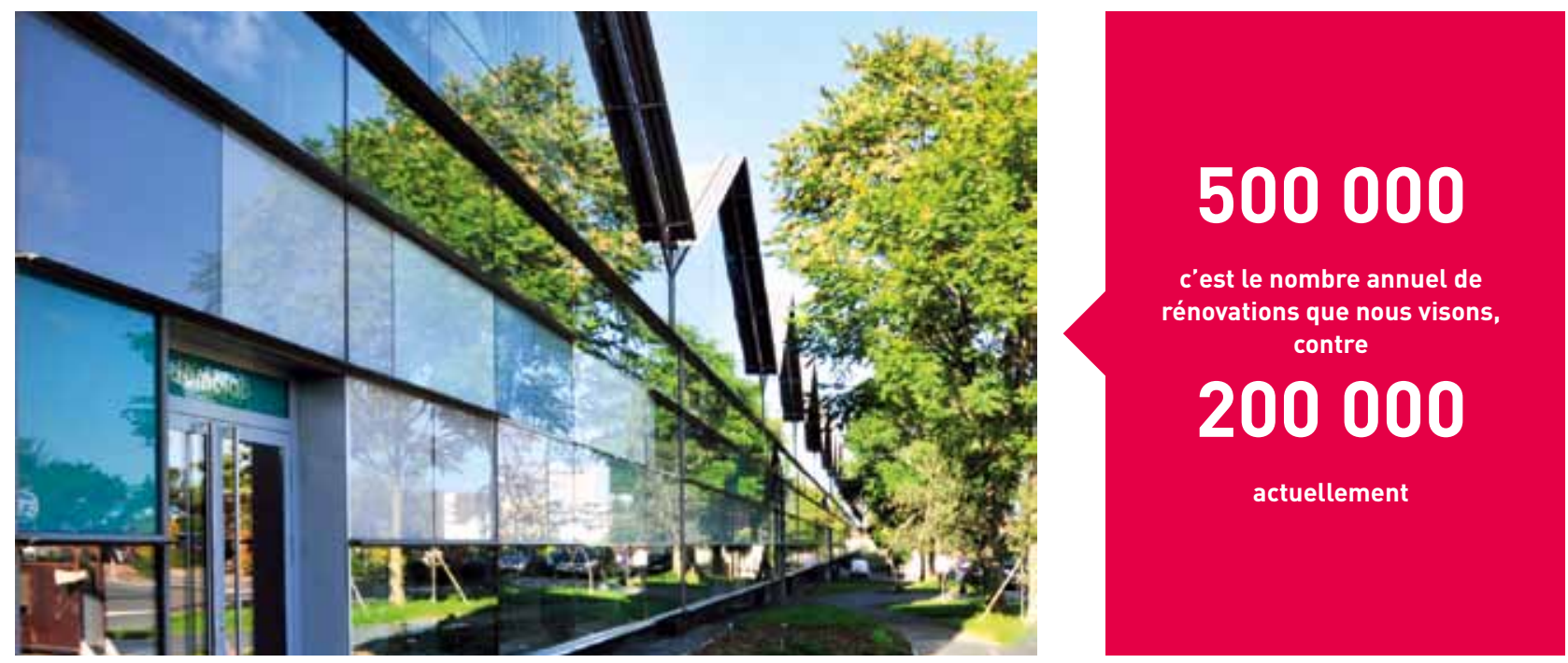

Domolab, premier centre d'innovation pour l'habitat, Aubervilliers

- Nous construisons la France de l'efficacité énergétique. Un tiers des émissions de $\mathrm{CO}_{2}$ produits sur le sol français proviennent du gaspillage énergétique des bâtiments. L'efficacité énergétique est un enjeu écologique, social et économique. - Les objectifs fixés par les pouvoirs publics en matière de performance énergétique et environnementale des bâtiments nécessitent une véritable mutation des acteurs du secteur. Une meilleure association entre l'industrie manufacturière et les professionnels du bâtiment est nécessaire. Elle permettra la mise au point d'offres industrialisées à la fois performantes et compétitives, et le développement de formations qualifiantes adaptées visant une réelle montée en compétence des professionnels du bâtiment sur le volet énergétique. - L'ambition du plan est de fédérer la filière, au niveau technique et organisationnel, pour qu'elle soit en mesure de proposer une offre globale de rénovation énergétique du bâtiment à des coûts maîtrisés supportables par les ménages. Notre objectif est de porter le nombre de rénovations d'environ 200000 par an actuellement, à 500 000. Le marché du bâtiment sera stimulé par de nouveaux outils tels que le fonds de garantie bancaire et le dispositif de tiers financeur. Le surcroît d'activité induit par ce plan engendrerait près de 75000 emplois. L'État apporte aux acteurs du plan ses propres leviers: la lisibilité et la simplicité des aides publiques, la mise en place du parcours de qualité avec un diagnostic de performance énergétique fiabilisé et la tierce vérification. - L'export doit enfin devenir un objectif de toute la filière pour stimuler le savoir-faire national, au contact des meilleures pratiques internationales et contribuer à l'emploi et au rééquilibrage de notre commerce extérieur. - La mise en place du plan s'inscrit dans un ensemble d'initiatives gouvernementales, dont le plan national de rénovation énergétique de l'habitat (PREH) et son label de qualité, le RGE, qu'il enrichit par la mobilisation des industriels. 


\section{ÉQUIPE DU PLAN}

CHEF DE PROJET

Dominique Maillard

est président du Directoire de RTE.

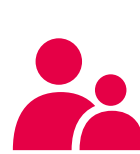

LES OPÉRATEURS DE RÉSEAUX

ERDF, RTE, URM

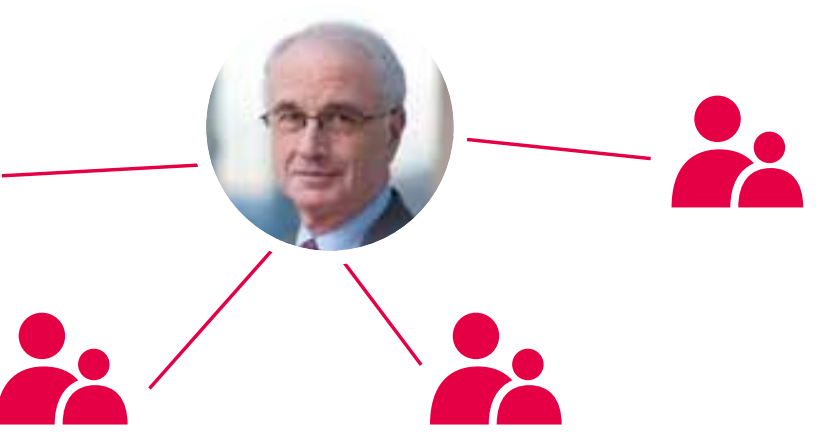

LES ACTEURS PUBLICS

MEIN, MEDDE, ADEME, CRE, CGI, Bpifrance

LES ÉCOLES, ORGANISMES DE RECHERCHE ET STRUCTURE D'ACCOMPAGNEMENT

CEA, Institut Carnot M.I.N.E.S,

pôles de compétitivité

Alcatel-Lucent, Capgemini, Sagemcom

SYNTHĖSE DES ACTIONS DU PLAN

\begin{tabular}{|c|c|c|}
\hline Action & Calendrier & Acteurs publics et levier d'action \\
\hline \multicolumn{3}{|c|}{ L'équipe de France des Réseaux électriques intelligents } \\
\hline \multirow[t]{3}{*}{$\begin{array}{l}\text { Création d'un groupement pour fédérer } \\
\text { la filière REI en France et organiser sa } \\
\text { promotion à l'international. }\end{array}$} & $\begin{array}{l}\text { Fin } 2014 \text { : Création du groupement } \\
\text { et établissement de l'annuaire de } \\
\text { la filière }\end{array}$ & MEIN et MEDDE associé au pilotage \\
\hline & $\begin{array}{l}\text { Renforcement de l'efficacité de } \\
\text { l'action française sur la normalisation }\end{array}$ & Rôle de facilitateur de l'État \\
\hline & $\begin{array}{l}2015 \text { : Mise en œuvre opérationnel } \\
\text { d'un réseau de vitrines du savoir- } \\
\text { faire, lancement d'un démonstrateur } \\
\text { à l'étranger }\end{array}$ & $\begin{array}{l}\text { Soutien de l'État à la visibilité de la filière (visites officielles, réseau } \\
\text { consulaire, UbiFrance...) et à l'innovation technologique, ou en qualité } \\
\text { d'investisseur avisé. }\end{array}$ \\
\hline \multirow{2}{*}{$\begin{array}{l}\text { Création d'une académie des REI pour } \\
\text { bâtir une offre de formation adaptée } \\
\text { aux enjeux de la filière }\end{array}$} & 2014 : Diagnostic & Pilotage de l'action par le réseau des Instituts Carnot \\
\hline & 2015 : Lancement de l'académie & \\
\hline \multicolumn{3}{|l|}{ De la démonstration à la réalisation } \\
\hline \multirow{2}{*}{$\begin{array}{l}\text { Organisation d'un déploiement ciblé } \\
\text { à grande échelle des réseaux } \\
\text { électriques intelligents en France }\end{array}$} & \multirow[t]{2}{*}{$\begin{array}{l}2014 \text { : Ciblage des zones prioritaires. } \\
2015 \text { : Lancement du projet }\end{array}$} & $\begin{array}{l}\text { Adaptation du cadre législatif pour une expérimentation poussée } \\
\text { (coordination par le MEDDE) }\end{array}$ \\
\hline & & $\begin{array}{l}\text { Sollicitation d'un soutien financier par les collectivités locales et les } \\
\text { Investissements d'avenir (50 à } 100 \mathrm{M} € \text { ) }\end{array}$ \\
\hline \multirow{2}{*}{$\begin{array}{l}\text { Mise en place sur des campus } \\
\text { universitaires un réseau électrique } \\
\text { intelligent à but de formation } \\
\text { et de recherche }\end{array}$} & \multirow{2}{*}{$\begin{array}{l}2014 \text { : Rédaction d'un cahier des } \\
\text { charges et appel à candidature } \\
2015 \text { : Lancement du/des projets }\end{array}$} & \multirow{2}{*}{$\begin{array}{l}\text { Pilotage par le CEA } \\
\text { Soutien financier pour le déploiement d'un réseau expérimental. }\end{array}$} \\
\hline & & \\
\hline \multicolumn{3}{|c|}{ Prendre de l'avance dans la course à l'innovation } \\
\hline $\begin{array}{l}\text { Définition de la stratégie R\&D } \\
\text { de la filière REI }\end{array}$ & $\begin{array}{l}2015 \text { : Identification de priorités R\&D } \\
\text { et structuration en réseau pour créer } \\
\text { des consortia répondant à des appels } \\
\text { à projets }\end{array}$ & Pilotage par les Instituts Carnot \\
\hline \multirow{2}{*}{$\begin{array}{l}\text { Organisation d'un concours d'idées } \\
\text { pour l'émergence et le déploiement } \\
\text { de solutions innovantes portées } \\
\text { par des start-ups }\end{array}$} & $\begin{array}{l}2014 \text { : Lancement d'un premier } \\
\text { concours d'idées par un opérateur } \\
\text { de réseau }\end{array}$ & \multirow[t]{2}{*}{$\begin{array}{l}\text { Soutien des initiatives pilotes retenues en appui des opérateurs de } \\
\text { réseau }\end{array}$} \\
\hline & $\begin{array}{l}2015-2016: \text { Lancement des } \\
\text { expérimentations }\end{array}$ & \\
\hline $\begin{array}{l}\text { Mise en place une structure } \\
\text { pour accompagner les start-ups }\end{array}$ & $\begin{array}{l}2014: \text { Mise en place des } \\
\text { démarches d'accompagnement; } \\
\text { démarrage des travaux sur les } \\
\text { blocages réglementaires limitant } \\
\text { l'industrialisation des innovations }\end{array}$ & $\begin{array}{l}\text { Participation au financement des coûts de fonctionnement des } \\
\text { pôles de compétitivité, participation aux réflexions sur les blocages } \\
\text { réglementaires }\end{array}$ \\
\hline
\end{tabular}




\section{RÉSEAUX ÉLECTRIQUES INTELLIGENTS}
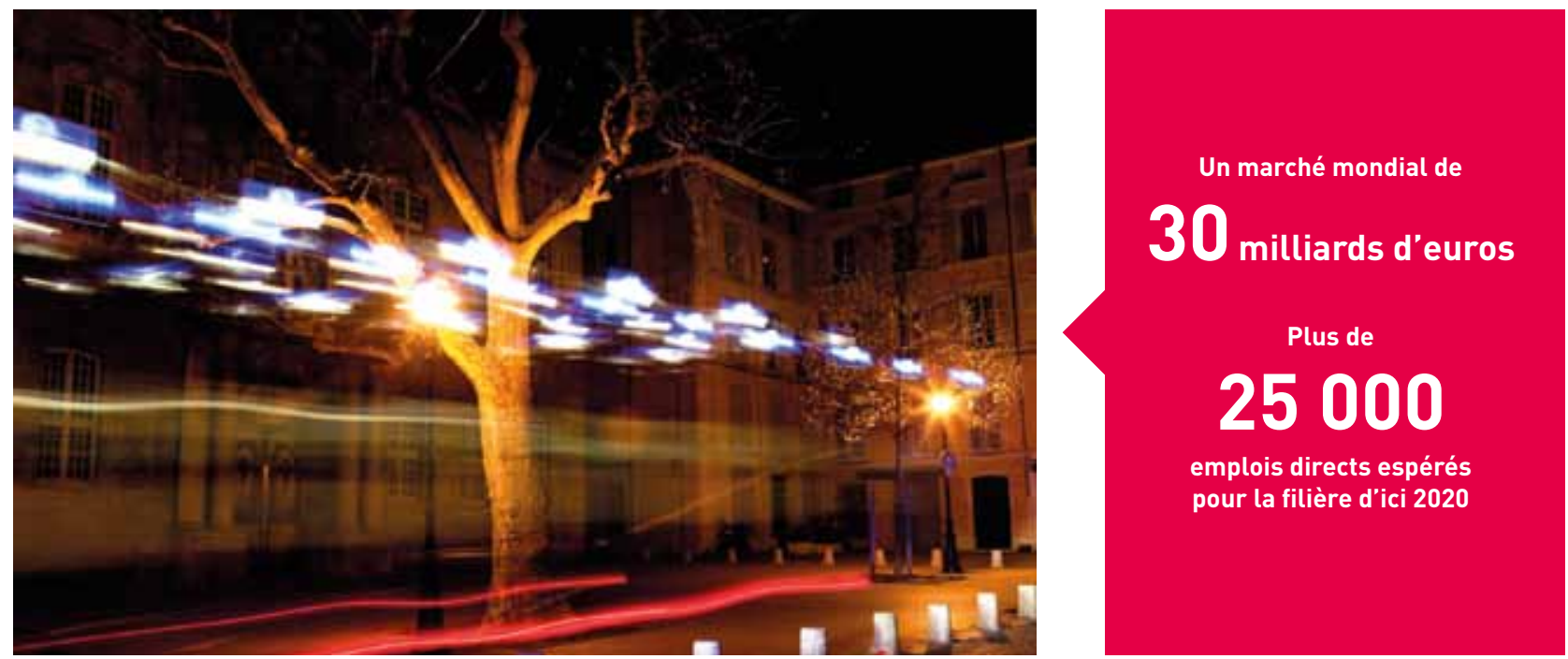

L'électricité au cœur de nos villes

- Nous construisons la France des réseaux électriques intelligents. Nos manières de consommer l'électricité se transforment, avec par exemple l'arrivée du véhicule électrique. Nos modes de production d'électricité évoluent, à la faveur du développement des énergies renouvelables et de notre volonté de réduire l'empreinte environnementale de nos activités. Ce sont de nouvelles contraintes pour le système électrique, qui doit évoluer pour maintenir une fourniture fiable d'électricité à faible émission de $\mathrm{CO}_{2}$ et à prix modéré. L'intégration des technologies de l'information et de la communication dans les réseaux peut y contribuer en créant des réseaux encore plus intelligents. Ils permettront aux producteurs d'électricité et aux opérateurs de réseaux d'adapter au plus juste les besoins d'investissement. Chaque consommateur pourra également adapter sa consommation, grâce notamment aux compteurs électriques intelligents, et être acteur du fonctionnement optimal du système électrique. - L'objectif de la filière française des réseaux électriques intelligents est de représenter d'ici 2020, plus de 25000 emplois directs en France pour un chiffre d'affaires d'au moins 6 milliards d'euros. Au croisement des transitions numérique et énergétique, le lancement du plan a vocation à consolider les filières électriques et informatiques et à en faire les chefs de file d'un secteur stratégique à forte croissance pour un marché annuel mondial de l'ordre de 30 milliards d'euros. - L'industrie française dispose d'atouts qu'il s'agit de valoriser dans la compétition internationale. Les trois axes majeurs du plan visent à amplifier et fédérer les efforts déjà engagés par l'État et les industriels. À court terme, le plan veut réunir les acteurs de la filière autour d'un «label» et d'une structure opérationnelle pour créer une «équipe de France des réseaux électriques intelligents» qui se positionnera comme un leader mondial capable d'exporter le savoir-faire français. À moyen terme, le plan permettra de passer des démonstrateurs à un déploiement industriel ciblé sur une zone géographique en France, pour concentrer les efforts, créer un véritable bassin d'emploi et rendre visible les atouts de la filière. À long terme, le plan préparera la compétitivité de la filière à l'horizon 2020, en peaufinant la stratégie R\&D et en favorisant l'émergence de solutions innovantes imaginées par les PME et les start-ups, notamment grâce à un concours d'idées. 


\title{
ÉQUIPE DU PLAN
}

\author{
CHEFS DE PROJET
}

Jean-Louis Chaussade est directeur général du groupe Suez Environnement. Christophe Chevillion est PDG du groupe Environnement SA.

LES ACTEURS PUBLICS DGCIS, MEIN, MEDDE, MENESR, MEAS, SIAAP, Agence de l'Eau Rhône Méditerranée et Corse, Bpifrance, COSEI Eau, les Régions (Centre, LanguedocRoussillon, Midi-Pyrénées et Alsace)
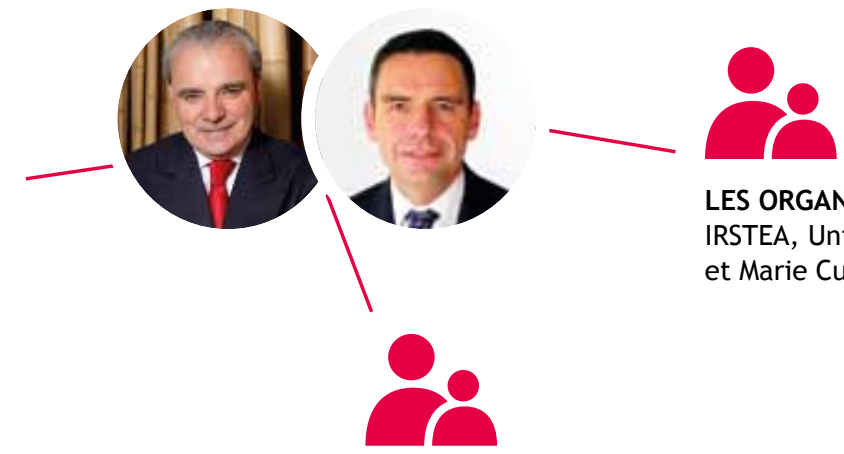

LES ORGANISMES DE RECHERCHE IRSTEA, Université Pierre et Marie Curie

\section{LES INDUSTRIELS}

Environnement SA, SUEZ Environnement, Véolia Environnement, Vinci Environnement, Areva, Saint Gobain Pam, ANTEA Group, ARTELIA, BIO-UV, Bordas UV GERMI, BRL Ingénierie, Électronique

SAS, Eseco Systems, ECOFILAE, IRH Environnement, Pôle EAU, Pôle HYDREOS, Pôle Mer Bretagne, Pôle DREAM, POLYMEM, Ponsel, PROFLUID, SAFEGE, Sappel, SOURCES, ITRON France, TERLI, MDB

TEXINOV, SAINTE LIZAIGNE SA, SAPPEL SAS, Swelia, et bien d'autres

\section{SYNTHĖSE DES ACTIONS DU PLAN}

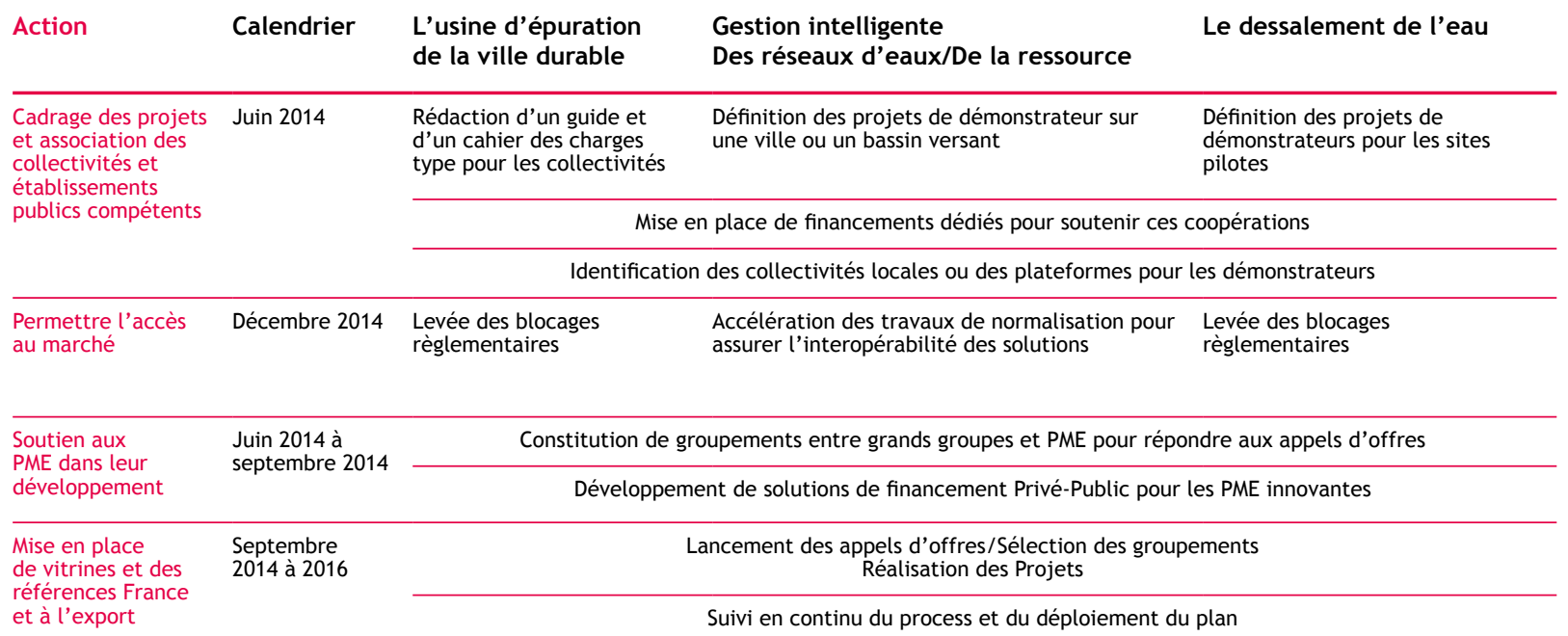




\section{QUALITÉ DE L'EAU ET GESTION DE LA RARETÉ}
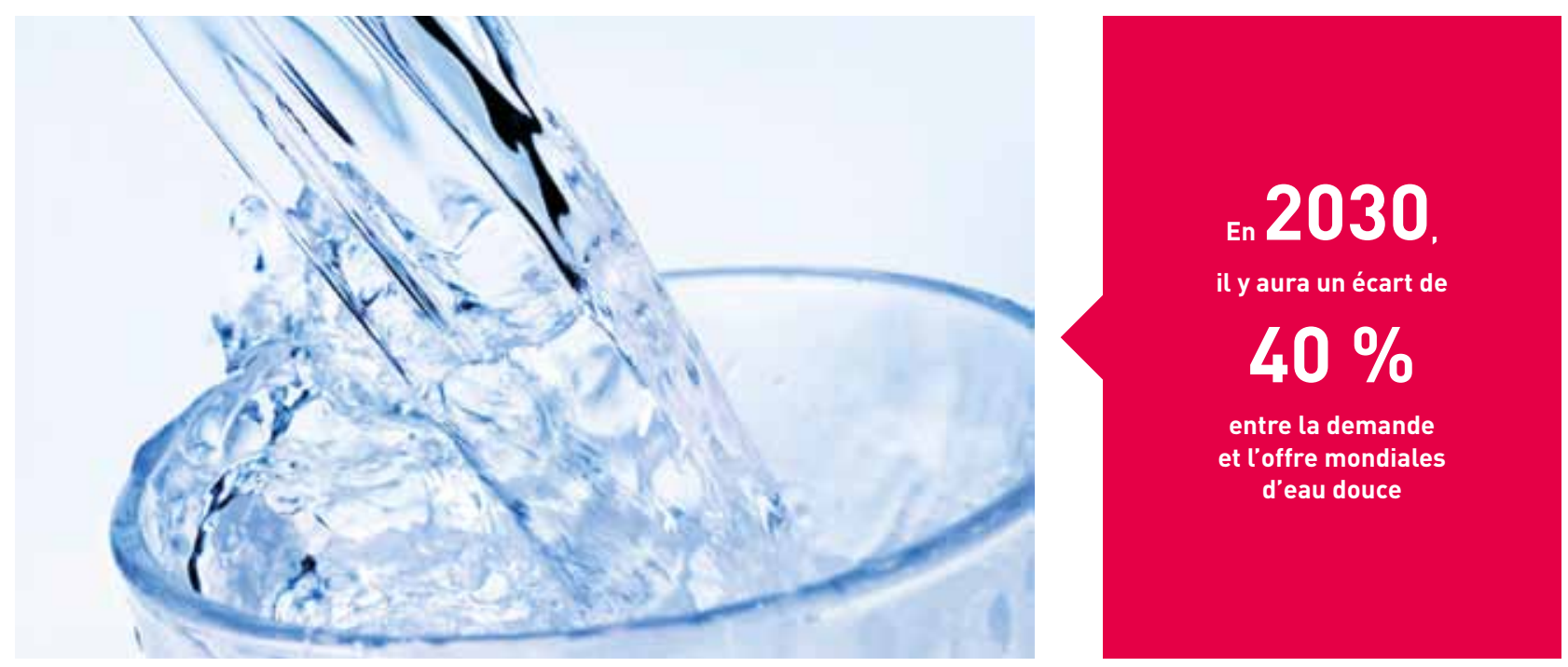

L'eau, ressource précieuse

Nous construisons la France de la gestion intelligente de l'eau. L'approvisionnement en eau est l'un des grands défis sociaux, politiques et économiques auxquels le monde est aujourd'hui confronté. En 2030, il y aura un écart de 40 \% entre la demande et l'offre mondiales d'eau douce, si les méthodes de gestion de l'eau n'évoluent pas vers une plus grande efficacité de production et de distribution. En 2014, la pénurie d'eau se classe troisième dans le Rapport mondial des Risques du Forum économique mondial sur les risques les plus préoccupants. - Face à ce défi, la France possède un savoir-faire et une expertise unique à l'échelle mondiale, ayant pour principal moteur une très forte capacité de recherche et d'innovation. Ce plan vise à soutenir l'effort d'innovation dans la filière eau, à conforter la position de leader dans le monde et à adapter les développements industriels aux enjeux de demain. Nous organisons l'interaction entre la capacité de grands groupes français à mener des projets innovants de grande ampleur, le dynamisme et la spécialisation de petites et moyennes entreprises françaises, ainsi que la volonté de l'État et des régions à stimuler le tissu économique. - L'effort se concentre ainsi autour de quatre axes de développement clé de l'offre industrielle: les usines d'épuration du futur à faible empreinte environnementale ; le grand cycle de l'eau, notamment face à l'adaptation au changement climatique ; le petit cycle de l'eau pour mieux limiter les pertes de ressources en eau sur le réseau et contrôler la qualité de l'eau ; le dessalement de l'eau, en vue de développer une offre à l'export. - La mise en œuvre de ce plan fera appel à la capacité de déploiement de l'innovation par la commande publique pour les innovations en matière de station d'épuration, de petit cycle de l'eau, par l'ouverture de certaines possibilités d'expérimentation (usages des eaux usées traitées notamment), par le soutien de l'État pour le passage de la preuve de concept à l'industrialisation pour des briques technologiques clés. Ces projets technologiques seront une vitrine du savoir-faire français pour le marché interne mais aussi et surtout pour l'export. 


\title{
ÉQUIPE DU PLAN
}

\author{
CHEF DE PROJET
}

Pascal Barthélemy

est directeur général adjoint de l'IFP Énergies nouvelles.

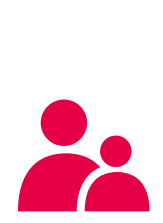

LES INDUSTRIELS

Sofiproteol, Solvay, Total,

Novacap, UIC, PCAS

Arkema, Roquette

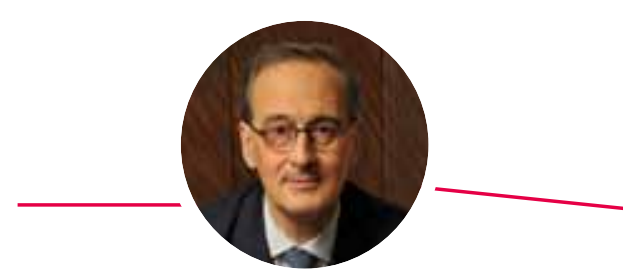

LES ACTEURS PUBLICS

CGI, MAAF, MENESR, MEIN, MEDDE, Ademe, DGCIS, BPI

\section{SYNTHÈSE DES ACTIONS DU PLAN}

\author{
Action \\ Pilote \\ Calendrier
}

Accompagnement par la puissance publique de projets d'investissement industriels pour la chimie verte et les biocarburants à partir du seconnd semestre 2014

Projets réduisant l'impact environnemental de la chimie et apportant aux autres secteurs des solutions pour réduire leur empreinte

Amélioration des procédés de la chimie, en particulier avec l'utilisation des biotechnologies

Production d'énergie plus performante (chaudières biomasse, méthanisation)

et amélioration de l'efficacité énergétique

Utilisations de matières premières recyclées

SNF, Adisseo,

2014 à 2018

Sofiproteol, Deinove,

PPC, Total, Bluestar,

Arkema, Cristal Union

Enersens Novacap,

Production de nouveaux matériaux pour répondre aux besoins de la transition

énergétique et de la lutte contre le changement climatique (gaz fluorés de

$4^{\mathrm{e}}$ génération, filière industrielle de la super isolation, etc.)

\section{Projets développant l'utilisation des ressources végétales}

Production de polymères biosourcés, destinés à des applications diverses:

peintures, emballages biodégradables, traitement des eaux, etc.

Baikowski, Minathiol..

Production de molécules biosourcées à forte valeur ajoutée

butadiène produit à partir d'éthanol, molécules pour des applications spécifiques

(additifs pour les plastiques, tensio-actifs...)

Projets développant les biocarburants avancés en complément des biocarburants de première génération

Production de biocarburants à partir de matières premières produites

en interculture (période séparant la récolte de la culture suivante)

Développement de la filière de production de biocarburants avancés :

biodiesel à base de déchets, éthanol et biodiesel 2G, biométhane

Mise en place d'un contexte favorable au développement de la chimie verte et des biocarburants

Favoriser le développement des marchés aval pour la chimie verte basée

sur des ressources végétales:

- Donner un accès privilégié aux marchés publics pour les produits biosourcés

- Mettre en place une fiscalité plus avantageuse (bonus vert) pour les produits biosourcés

la production des biocarburants de première génération

Faciliter l'accès aux ressources d'origine végétale à un prix compétitif tout en préservant les intérêts des agro-industries
Sofiproteol, GDF Suez, 2014 à 2019

MEDDE, ADEME, MEIN

Budget, MEIN, MEDDE

Groupe de travall avec

Groupe de travail avec MEIN, MAAF, MEDDE

SNF, Sekab, Solvay, Total, Cristal Union,

Salveco, Michelin,

2014 à 2020

Groupe de travail avec MEIN, MAAF
Benchmark: 2e semestre 2014

Proposition : 2015

Proposition : 2015

Décision: 2016

Mise en œuvre : 2016

Propositions de mesures : fin 2014

Propositions de mesures :

1 er trimestre 2015 


\section{CHIMIE VERTE ET BIOCARBURANTS}
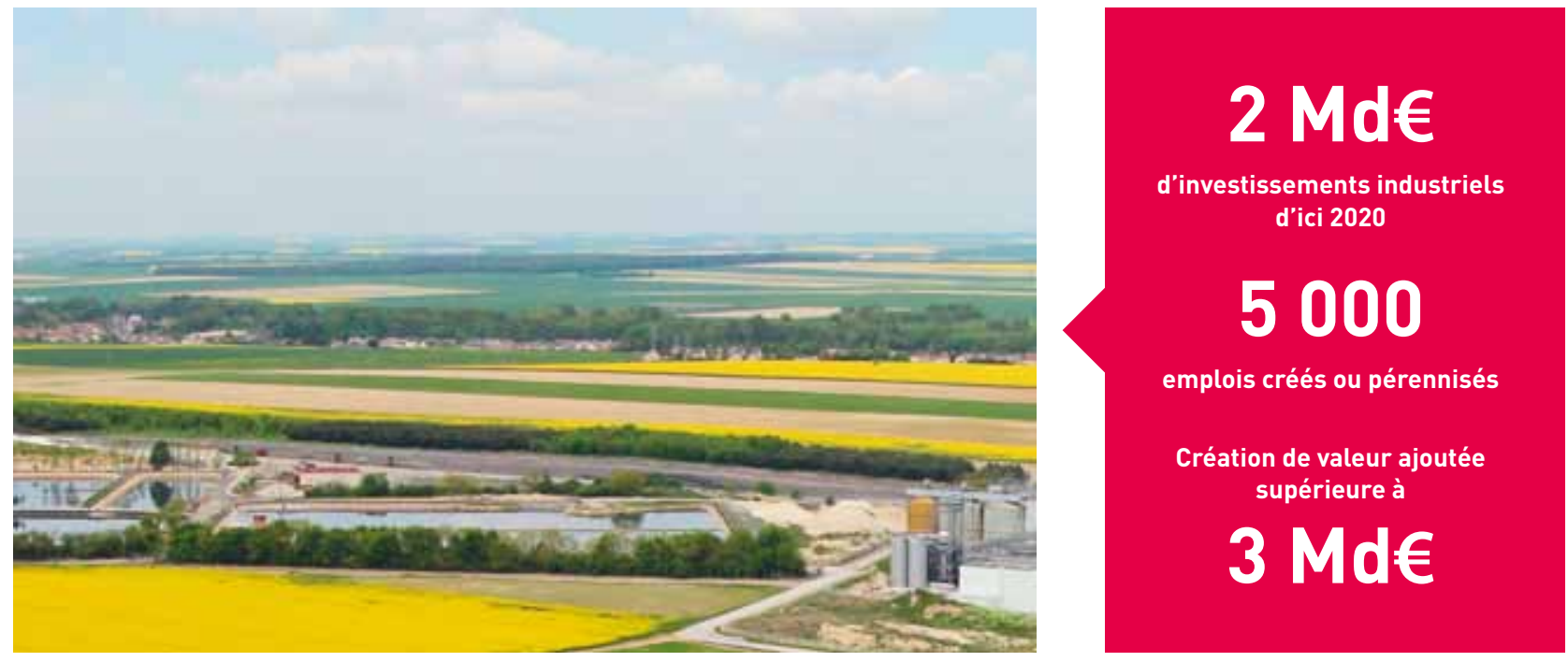

Usine de chimie végétale, transformation du colza

- Nous construisons la France des molécules vertes où la chimie anticipe l'après-pétrole. Une industrie chimique performante, capable de s'adapter aux enjeux environnementaux, est indispensable pour répondre aux besoins des activités industrielles situées en aval, qu'il s'agisse de l'allègement des véhicules, de l'isolation des bâtiments ou du stockage de l'énergie. De manière similaire, l'industrie des biocarburants contribue à la lutte contre le changement climatique et à l'indépendance énergétique de la France. Ces secteurs tiennent donc une importance considérable dans l'économie nationale. - Il faut favoriser la conversion des entreprises pour qu'elles utilisent des procédés plus propres et moins consommateurs d'énergie. L'utilisation des ressources végétales doit également être soutenue, en cohérence avec les atouts dont dispose la France dans les domaines agricole et forestier, car elle offre des opportunités de développement industriel (production de nouveaux polymères ou de molécules à forte valeur ajoutée, par exemple pour le secteur de la cosmétique). $\rightarrow$ Le développement de la production de biocarburants avancés, basés sur l'utilisation de déchets ou de la biomasse lignocellulosique, représente un enjeu important pour répondre aux exigences qui se mettent en place au niveau de l'Union européenne. Les entreprises qui s'engagent dans cette voie doivent cependant maintenir la production des biocarburants de première génération dans laquelle elles ont déjà investi. • L'élaboration de la feuille de route a conduit à identifier plus de 40 projets d'investissement industriels, à des niveaux de maturation plus ou moins avancés, mais pour lesquels des difficultés empêchent la décision d'investissement. Ces difficultés sont de différents ordres (financier, réglementaire, normatif, etc.). — L'État accompagnera ces projets d'investissement en analysant les difficultés rencontrées et en s'attachant à proposer des solutions. Des actions seront menées en parallèle par l'État pour mettre en place un contexte favorable au développement de la chimie verte et des biocarburants. 


\section{ÉQUIPE DU PLAN}

CHEF DE PROJET

André Choulika est président de Cellectis.

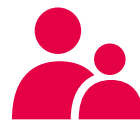

LES OPÉRATEURS

Erytech, Theravectys,

Sanofi, Ose Pharma Truffle Capital

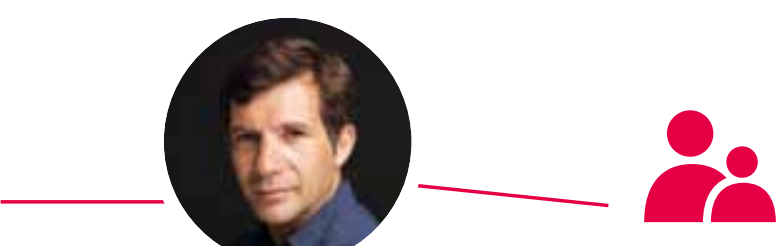

LES ACTEURS PUBLICS

Inserm pour MENESR, MASS, CGI, Bpifrance, INPI, DGCIS

\section{AVANCÉES THÉRAPEUTIQUES ET INDUSTRIELLES GÉNÉRÉES PAR LE PLAN}

\begin{tabular}{lll} 
Domaines & Calendrier & Finalités/livrables \\
\hline Cancer & $2015-2018$ & $\begin{array}{l}\text { Obtenir } 3 \text { preuves de concept et premières mondiales } \\
\text { Obtenir } 2 \text { mises sur le marché de nouveaux traitements } \\
\text { Obtenir au moins } 2 \text { nouveaux anticorps immunomodulateurs }\end{array}$ \\
\hline Maladies auto-immunes & $2016-2020$ & Preuve de concept dans le développement d'un médicament contre la maladie de Crohn \\
\hline Régénération & 2016 & $\begin{array}{l}\text { Première mondiale : la France met sur le marché un médicament de thérapie cellulaire } \\
\text { pour la régération du myocarde }\end{array}$ \\
\hline Vaccins thérapeutiques & $2015-2020$ & $\begin{array}{l}\text { La France renoue avec le leadership en termes de vaccins en développant un vaccin } \\
\text { thérapeutique contre l'hépatite B et un vaccin contre la bactérie responsable } \\
\text { des maladies nosocomiales }\end{array}$ \\
\hline Industrialisation & $\begin{array}{l}\text { La capacité de la France est renforcée par la construction d'un nouveau site industriel } \\
\text { dédié aux immunothérapies cellulaires }\end{array}$
\end{tabular}

\section{SYNTHÈSE DES ACTIONS DU PLAN}

\begin{tabular}{|c|c|c|c|c|}
\hline Action & Pilote & $\begin{array}{l}\text { Acteurs publics } \\
\text { et levier d'action }\end{array}$ & Acteurs & Finalités/livrables \\
\hline Assurer des leviers de financement & CoPil du Plan & 2014-2018 & Bpifrance, CGI & $\begin{array}{l}\text { Transformer les résultats de la } \\
\text { recherche en activité industrielle }\end{array}$ \\
\hline Optimiser l'environnement financier & Truffle Capital & Dès 2014 & & $\begin{array}{l}\text { Faciliter la croissance des } \\
\text { entreprises et leur investissement } \\
\text { en R\&D }\end{array}$ \\
\hline Adapter les contraintes réglementaires & CELLforCURE & 2015 & $\begin{array}{l}\text { ANSM, HAS CEPS, } \\
\text { DGCIS, LEEM, } \\
\text { EMaA }\end{array}$ & $\begin{array}{l}\text { Faciliter la mise en place d'essais } \\
\text { cliniques et accélérer l'accès au } \\
\text { marché }\end{array}$ \\
\hline $\begin{array}{l}\text { Favoriser le déploiement } \\
\text { à l'international }\end{array}$ & CELLECTIS & 2015-2016 & $\begin{array}{l}\text { Ubifrance, } \\
\text { COFACE, } \\
\text { Bpifrance } \\
\text { International }\end{array}$ & $\begin{array}{l}\text { Simplifier l'import-export de } \\
\text { matières premières et produits } \\
\text { biologiques et appuyer les } \\
\text { industriels à l'export }\end{array}$ \\
\hline Développer la Propriété Industrielle & $\begin{array}{l}\text { INPI, France } \\
\text { Brevets }\end{array}$ & $2014-2015$ & $\begin{array}{l}\text { INPI, France } \\
\text { Brevets }\end{array}$ & $\begin{array}{l}\text { Faire bénéficier l'Equipe de France } \\
\text { des Thérapies Innovantes } \\
\text { d'une approche globale en matière } \\
\text { de propriété industrielle }\end{array}$ \\
\hline
\end{tabular}




\section{BIOTECHNOLOGIES MÉDICALES}
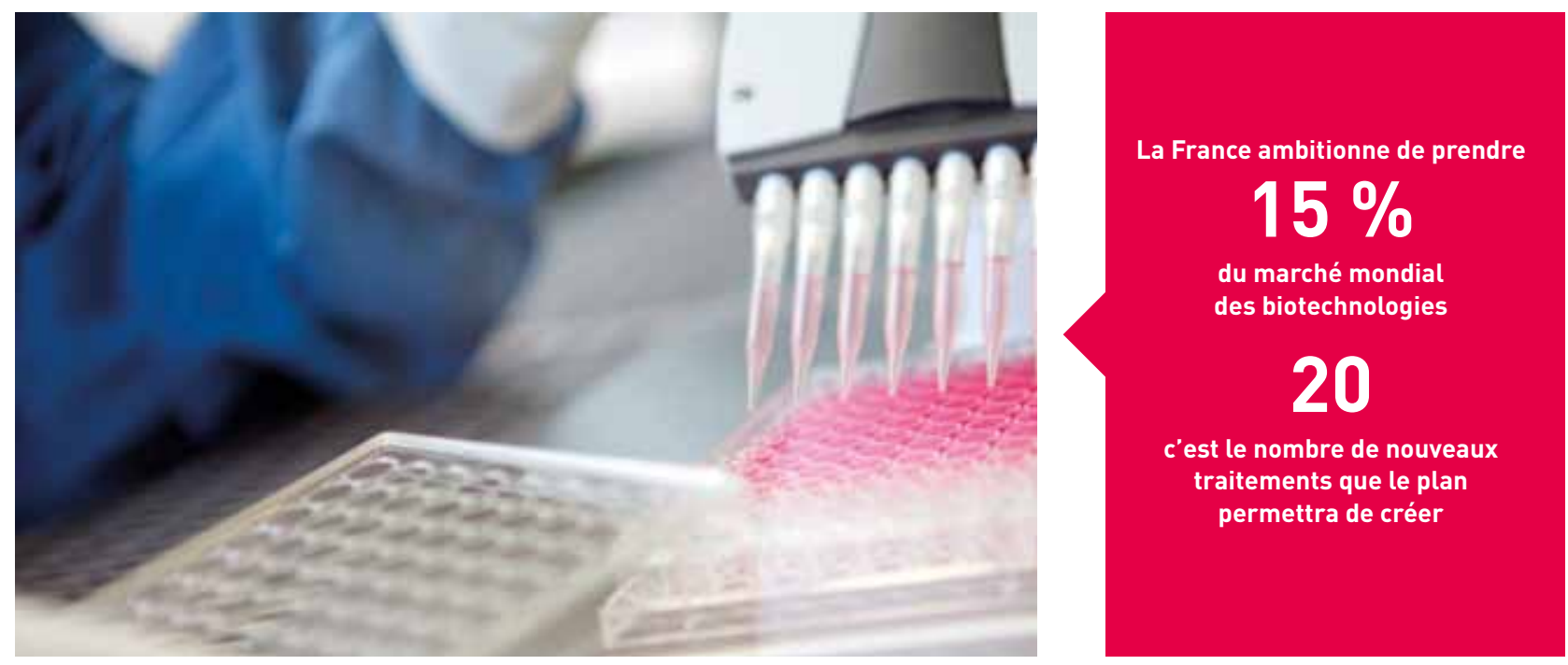

Laboratoire de biotechnologies, France Biotech

Nous construisons la France des thérapies innovantes. Le plan «Biotechnologies médicales» vise à renforcer les positions françaises en matière de thérapie cellulaire, immunitaire et vaccinale. Aujourd'hui, sur un marché pharmaceutique mondial de plus de 1150 milliards de dollars, $70 \%$ des produits pharmaceutiques en développement sont des biomédicaments. La France, historiquement en pointe dans le domaine des médicaments issus de la chimie, prend le virage des biotechnologies en se positionnant sur un sujet d'avenir : les médecines cellulaires. - Notre pays peut compter sur une recherche de premier rang dans certains domaines de pointe, sur des capacités de bioproduction importantes ainsi que sur un écosystème de PME de haute technologie qui représentent à elles seules plus de 9000 emplois. Le plan déploie un programme ambitieux, exécuté par une Equipe de France industrielle s'appuyant sur les atouts de notre pays dans ce domaine compétitif. Il vise à structurer la filière autour d'acteurs clefs et de capacités industrielles. Il s'agit notamment de faire évoluer l'environnement, l'écosystème et l'attractivité de notre territoire pour ces développements de technologie de pointe. - Le plan entreprend des grandes premières mondiales fondées sur des innovations de rupture, ciblant, entre autres, 6 nouveaux vaccins, 10 nouveaux médicaments contre le cancer, 2 traitements d'affections immunitaires, la régénération du myocarde et de la rétine, et la construction en France d'une capacité industrielle unique dédiée aux thérapies cellulaires. - La France a tous les atouts pour prendre une place de leader sur l'un des grands gisements de croissance des années et décennies à venir amené à constituer un marché de 100 Md€ d'ici 15 ans. Le plan «Biotechnologies médicales » se focalise sur les étapes clefs de l'industrialisation et du développement clinique, de nature à ancrer savoir-faire, emplois et capacités au sein du territoire. 


\section{ÉQUIPE DU PLAN}

CHEFS DE PROJET

Mireille Faugère est conseiller maître à la Cour des Comptes, ancienne directrice générale de l'AP-HP.

Christian Nibourel est PDG d'Accenture France.

LES ACTEURS PUBLICS GCS1 Île-de-France, APHP,

CH Eaubonne-Montmorency,

$\mathrm{CHU}^{2}$ Montpellier, MASS, MENESR, MEIN

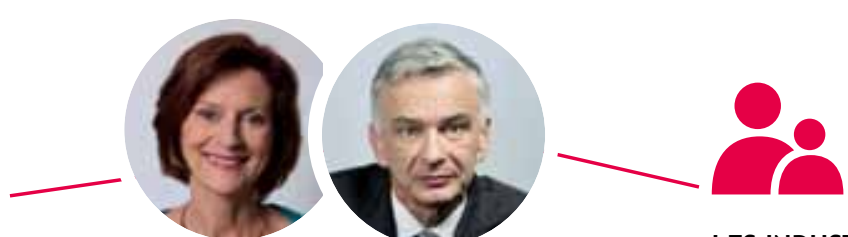

LES INDUSTRIELS

Bull, Orange, Voluntis, Syntec, Accenture

\section{SYNTHÈSE DES ACTIONS DU PLAN}

\begin{tabular}{|c|c|c|c|c|}
\hline Action & Pilote & Calendrier & Acteurs privés concernés & Finalités/livrables \\
\hline \multicolumn{5}{|c|}{ Des solutions industrielles de partage de données pour les professionnels de santé et les patients } \\
\hline $\begin{array}{l}\text { Création d'un cadre } \\
\text { opposable pour } \\
\text { l'échange de données, } \\
\text { des procédures } \\
\text { d'hébergement } \\
\text { simplifiées et } \\
\text { reconnaissance du NIR } \\
\text { comme identifiant } \\
\text { unique du patient } \\
\end{array}$ & MASS & $\begin{array}{l}\text { Premier trimestre } \\
2015 \text { pour la } \\
\text { création du cadre } \\
2015-2017 \text { pour } \\
\text { la reconnaissance } \\
\text { du NIR }\end{array}$ & $\begin{array}{l}\text { Éditeurs et intégrateurs, } \\
\text { hébergeurs, professionnels } \\
\text { de santé } \\
\text { Instances représentatives } \\
\text { des industriels du logiciel, } \\
\text { en relation étroite avec les } \\
\text { pilotes }\end{array}$ & $\begin{array}{l}\text { Travail sur les référentiels (identification, hébergement, } \\
\text { sécurité, interopérabilité, urbanisation) Simplification } \\
\text { législative permettant à l'État de rendre opposable les } \\
\text { référentiels élaborés par l'ASIP-Santé et de s'assurer de } \\
\text { leur respect } \\
\text { Extension progressive de l'éligibilité au suivi par le NIR à } \\
\text { tous les patients grâce aux mesures de la loi santé votée } \\
\text { en } 2015\end{array}$ \\
\hline $\begin{array}{l}\text { Renforcement } \\
\text { de la représentation } \\
\text { de la France dans } \\
\text { la normalisation }\end{array}$ & $\begin{array}{l}\text { MASS (DSSIS) } \\
\text { et MEIN } \\
\text { (DGCIS) ASIP } \\
\text { Santé ou } \\
\text { AFNOR }\end{array}$ & 2015 & $\begin{array}{l}\text { Chercheurs, industriels et } \\
\text { leurs instances représentatives } \\
\text { (SYNTEC, FIEEC...), } \\
\text { associations (PHAST...) }\end{array}$ & $\begin{array}{l}\text { Création d'une instance coordinatrice relayée dans } \\
\text { les fédérations industrielles, définissant les objectifs } \\
\text { prioritaires et assurant remontée des besoins et suivi des } \\
\text { actions de représentation }\end{array}$ \\
\hline \multicolumn{5}{|c|}{ Développer des services autour des parcours de soin et de la prévention, en commençant par les maladies chroniques } \\
\hline $\begin{array}{l}\text { Amplification de } \\
\text { l'utilisation des outils } \\
\text { numériques dans les } \\
\text { parcours de soin }\end{array}$ & $\begin{array}{l}\text { MASS(DGOS } \\
\text { et ARS) }\end{array}$ & 2016-2020 & $\begin{array}{l}\text { Ensemble des acteurs se } \\
\text { positionnant sur le marché de } \\
\text { la coordination de soins }\end{array}$ & $\begin{array}{l}\text { Extension progressive du périmètre des projets pilotes } \\
\text { TSN pour préparer un déploiement à grande échelle et } \\
\text { travailler à l'extension de la couverture en nombre de } \\
\text { patients } \\
\text { Développement des services d'appui à la coordination des } \\
\text { soins pour traiter } 700000 \text { cas très complexes et } 2000000 \\
\text { de cas complexes en } 2020 \text { dans des parcours de soin }\end{array}$ \\
\hline $\begin{array}{l}\text { Développement } \\
\text { des solutions de } \\
\text { télésurveillance } \\
\text { des maladies chroniques } \\
\text { en France }\end{array}$ & $\begin{array}{l}\text { MASS (DGOS } \\
\text { et ARS) }\end{array}$ & $2017-2020$ & $\begin{array}{l}\text { Industriels porteurs des } \\
\text { projets pour les thématiques } \\
\text { prioritaires choisies, sociétés } \\
\text { savantes, ordres et syndicats }\end{array}$ & $\begin{array}{l}\text { Emergence d'un marché des solutions de télésurveillance } \\
\text { des maladies chroniques (objectif de } 50000 \text { patients } \\
\text { suivis à horizon } 2017 \text { dans } 4 \text { domaines thérapeutiques } \\
\text { prioritaires, } 1000000 \text { patients en 2020). }\end{array}$ \\
\hline $\begin{array}{l}\text { Rendre le processus } \\
\text { réglementaire actuel } \\
\text { de prise en charge des } \\
\text { dispositifs médicaux et } \\
\text { des actes associés plus } \\
\text { prévisible et plus rapide }\end{array}$ & MASS & 2015 & Industriels de santé & $\begin{array}{l}\text { Simplification des procédures d'évaluation des dispositifs } \\
\text { médicaux (révision du «forfait innovation», leviers } \\
\text { législatifs au sein de la loi santé) }\end{array}$ \\
\hline \multicolumn{5}{|c|}{ Travailler sur la gouvernance et la qualité de la santé numérique et développer de nouveaux champs, comme la médecine personnalisée } \\
\hline $\begin{array}{l}\text { Mise en place une action } \\
\text { fédératrice pour la } \\
\text { médecine personnalisée } \\
\text { s'appuyant sur une base } \\
\text { de données nationale }\end{array}$ & $\begin{array}{l}\text { Groupe de } \\
\text { travail CGI, } \\
\text { puis pilote } \\
\text { industriel }\end{array}$ & 2014 & & $\begin{array}{l}\text { Organisation d'un système d'information susceptible } \\
\text { d'être accessible par tous les acteurs impliqués, montage } \\
\text { d'un consortium national regroupant plusieurs projets } \\
\text { de recherche et des industriels, de plusieurs spécialités } \\
\text { différentes }\end{array}$ \\
\hline $\begin{array}{l}\text { Développement d'une } \\
\text { offre de SI de qualité, } \\
\text { au niveau des standards } \\
\text { internationaux }\end{array}$ & MASS (DSSIS) & 2014 & & $\begin{array}{l}\text { Accélérer le processus de labellisation des acteurs } \\
\text { SI hospitaliers } \\
\text { Favoriser l'émergence d'un nombre limité de SIS } \\
\text { « champions » mutualisés }\end{array}$ \\
\hline $\begin{array}{l}\text { Définition et mise en } \\
\text { œuvre d'une politique } \\
\text { nationale en matière } \\
\text { de santé numérique }\end{array}$ & MASS & 2015 & & $\begin{array}{l}\text { Unifier et clarifier la gouvernance des } \mathrm{SI} \text { en matière de } \\
\text { santé pour mettre en œuvre la feuille de route santé } \\
\text { numérique } \\
\text { Massifier les investissements consacrés à la transition } \\
\text { numérique pour une période de } 5 \text { ans }\end{array}$ \\
\hline
\end{tabular}




\section{SANTÉ NUMÉRIQUE}
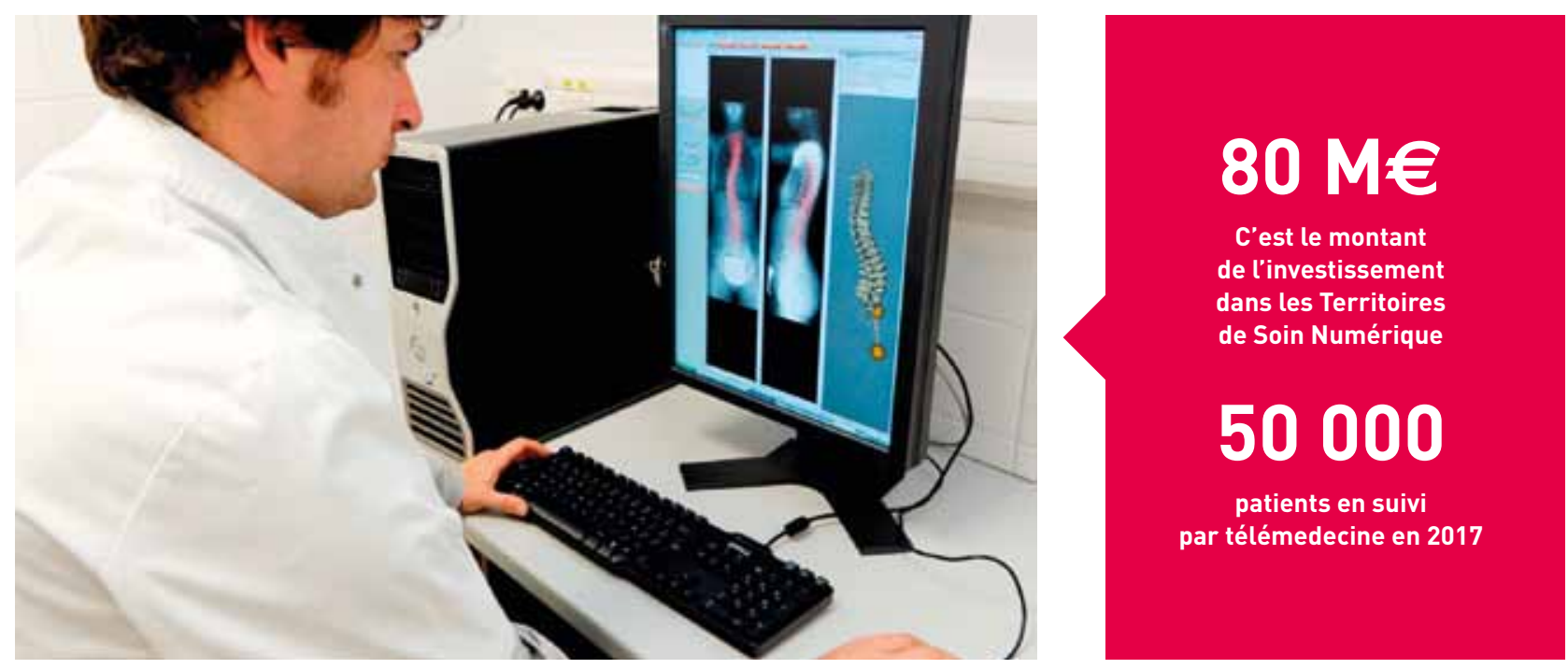

Imagerie médicale

- Nous construisons la France de la santé numérique.

Des dossiers médicaux électroniques à la télémédecine, la santé est entrée dans l'ère du numérique. La Stratégie Nationale de Santé identifie le numérique comme un levier essentiel de l'action publique pour combattre les injustices et les inégalités de santé et adapter le système de santé français aux profondes mutations qui sont à l'œuvre. Non seulement la santé numérique est une réalité, mais elle est en train de réinventer les modèles de santé. — Le plan a vocation à positionner la France comme le leader européen dans ce domaine. Il a été élaboré à la fois avec les fournisseurs de soins et les représentants de la filière qui mobilise un écosystème dynamique composé de grands industriels et de start-up. - Le marché français de la santé numérique atteint aujourd'hui 3 milliards d'euros, avec une croissance annuelle de l'ordre $5 \%$. Les actions majeures du plan visent à la fois à offrir de meilleurs soins aux patients, de l'innovation au service des professionnels de santé et une excellence industrielle pour toute la filière santé numérique. - Le plan permettra le développement de SI de qualité pour le continuum ville-hôpital autour des territoires de soin numériques et l'émergence d'un nombre limité de SI hospitaliers mutualisés, au niveau des standards internationaux. - À court terme, le déploiement du plan offrira un cadre réglementaire clair, simple et sécurisant de partage de données et de distribution de services pour les professionnels de santé et les patients. À moyen terme, le plan permettra le développement de la télésurveillance de maladies chroniques au-delà des expérimentations en cours pour soutenir les domaines d'excellence français et atteindre 50000 patients suivis en 2017. À long terme, le plan se fixe l'ambition d'étendre les services d'appui à la coordination de soins dans les parcours de soins de près de 700000 cas très complexes et 2000000 de cas complexes à l'horizon 2020. - L'État accompagnera ces efforts par la mise en œuvre d'une politique nationale en matière de santé numérique soutenue par une gouvernance unifiée, une trajectoire et des investissements massifiés. - Enfin, La France de la santé numérique s'engage également dans la voie prometteuse de la génomique et de la médecine personnalisée. 


\section{ÉQUIPE DU PLAN}

\section{CHEFS DE PROJET}

André-Michel Ballester est directeur général de Sorin Group. Sacha Loiseau est directeur général et fondateur de Mauna Kea Technologies.

LES ÉCOLES ET ORGANISMES DE RECHERCHE ITMO Technologies pour la Santé (AVIESAN)
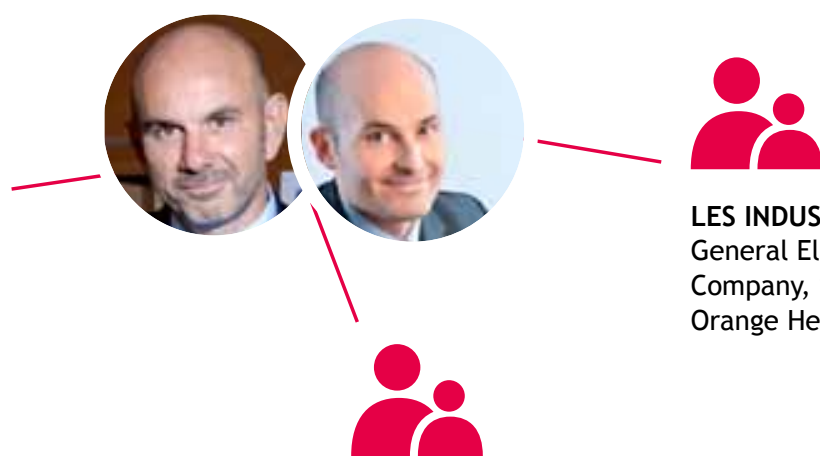

LES INDUSTRIELS

General Electric, Mc Kinsey \& Company, SOFINNOVA Partners, Orange Healthcare

LES ACTEURS PUBLICS

MEIN, MASS, AP-HP, CGI Bpifrance

\section{SYNTHĖSE DES ACTIONS DU PLAN}
Action
Pilote
Calendrier
Acteurs publics et leviers d'action

Accompagnement et accélération du développement des startups MedTech

Renforcement et soutien des incubateurs/

accélérateurs spécifiques

Soutien à des fonds d'entrepreneurs

spécialisés

Bpifrance, CGI Second semestre 2014

Création de MedTech Cities (mini-cluster)

regroupant les acteurs de l'écosystème

Medtech

\section{Consolidation des PME et ETI}

Identification des pilotes d'intégration

et réalisation des consolidations autour

d'eux

Accompagnement d'une opération de

consolidation par des dispositifs publics

pour maximiser l'effet de levier auprès

des financements privés

Réalisation d'une

$1^{\text {re }}$ consolidation d'ici

Organisation d'une journée de

valorisation des brevets académiques

avec les industriels de la filière ultrasons

et mise à disposition d'un panorama des

brevets des organisations publiques

début 2015

Bpifrance, CGI

201
Acteurs publics:

Bpifrance, CGI, Collectivités territoriales

Leviers:

Fonds French Tech pour co-investissement

Rapprochement d'entreprises françaises leaders, technologiquement complémentaires, autour de projets communs

Formation d'une Alliance des technologies

médicales (structure collaborative entre

grands groupes, ETI et PME) autour de

grands groupes,

Sélection d'un projet démonstrateur de

R\&D collaboratif fédérant grand groupe

Organisation de la journée de

Acteurs publics:

MEIN, Bpifrance, CGI, AVIESAN/COVALLIANCE

la valorisation en novembre

Leviers:

Intervention de Bpifrance en capital, prêts, garanties de

prêt, avec ses outils d'intervention: fonds Large Venture, fonds ETI 2020, etc.

Création de l'Alliance fin 2014

Sélection d'un projet de R\&D au 1 er trimestre 2015
Acteurs publics:

Bpifrance, CGI

Leviers:

Mobilisation des outils de soutien à l'innovation: PSPC, FUI, PIPC

Accompagnement du développement à l'international des entreprises

Simplification et raccourcissement

des procédures d'accès au marché des

technologies médicales

Utilisation des achats hospitaliers comme

levier de développement des entreprises

innovantes

Fin 2014

Mise en place d'un outil favorisant les

transferts de technologies de la recherche

academique et hospitalière vers la filière

industrielle
Démarrage l'outil de transfert dernier trimestre 2014
Levier:

Publication de textes réglementaires et législatifs, inspirés notamment des propositions du CSF industries et technologies de sante 


\section{DISPOSITIFS MÉDICAUX ET NOUVEAUX ÉQUIPEMENTS DE SANTÉ}
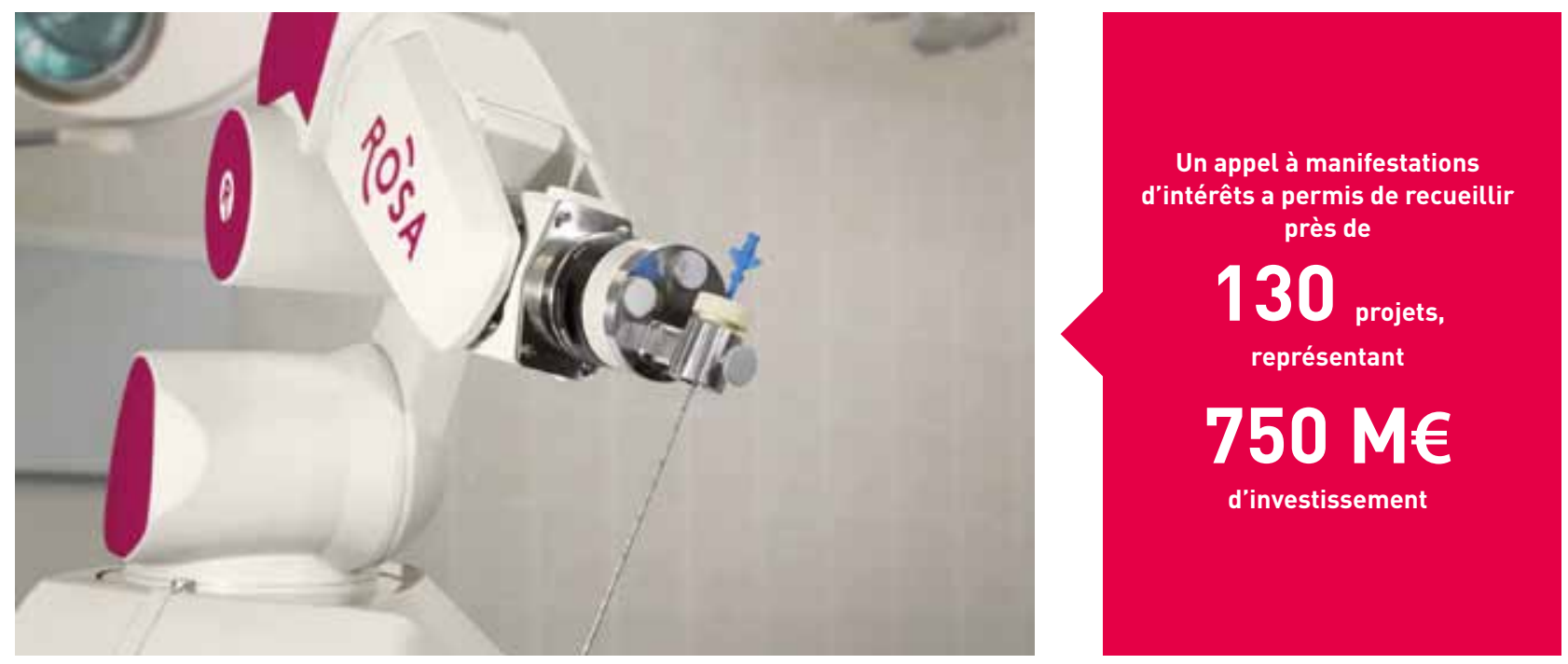

Robot Rosa d'assistance aux gestes chirurgicaux, Medtech

- Nous construisons les dispositifs médicaux et les équipements de santé de la France de demain. Des équipements d'imagerie médicale (scanner, IRM, échographes.), jusqu'aux dispositifs médicaux implantables (stents, pacemakers, valves cardiaques etc.), en passant par les dispositifs de diagnostic in vitro, les implants orthopédiques, les textiles médicaux ou encore les logiciels informatiques, les opportunités sont vastes. - Grâce au dynamisme de nombreuses PME et de quelques ETI, et grâce à une recherche scientifique et technologique internationalement reconnue, la France a les atouts pour faire naître et produire sur son territoire les technologies médicales innovantes capables de conquérir les marchés étrangers. Au niveau mondial, le marché des dispositifs médicaux est estimé à 300 milliards d'euros et il connaît un rythme annuel de croissance de $4 \%$ à $5 \%$. Les domaines technologiques à mobiliser sont variés : électronique, pharmacie, mécanique, physique, informatique, chimie, plasturgie, optique. Les bénéfices potentiels pour les malades sont multiples, en particulier lorsqu'il s'agit d'accompagner leur vieillissement. Le plan dispositifs médicaux et nouveaux équipements de santé permettra d'accélérer le développement de startups, de consolider les PME et ETI qui ont du mal à relever les défis réglementaires et cliniques du secteur, de rapprocher les entreprises appartenant à des secteurs technologiques complémentaires autour de projets communs et enfin d'aider au développement d'une offre française à l'international. Sous l'égide du Conseil National de l'Industrie, des États Généraux de la filière dispositifs et technologies médicales seront régulièrement tenus dès 2015, pour suivre la mise en œuvre de la feuille de route et traiter de sujets spécifiques, notamment en matière de financement privé et public. 


\section{ÉQUIPE DU PLAN}

CHEF DE PROJET

Jean-Philippe Girard est PDG d'Eurogerm.

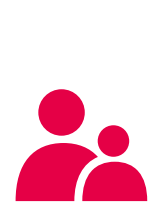

LES INDUSTRIELS

Eurogerm, Pernod Ricard, Sofiprotéol, Tereos, Copalis, Isigny Sainte Mère, LimagrainURIAA Auvergne, ANIA, Coop de

France, Food For Life France,

Fédération des Industries Mécaniques, Comité de Liaison des Industriel Francais de l'Emballage

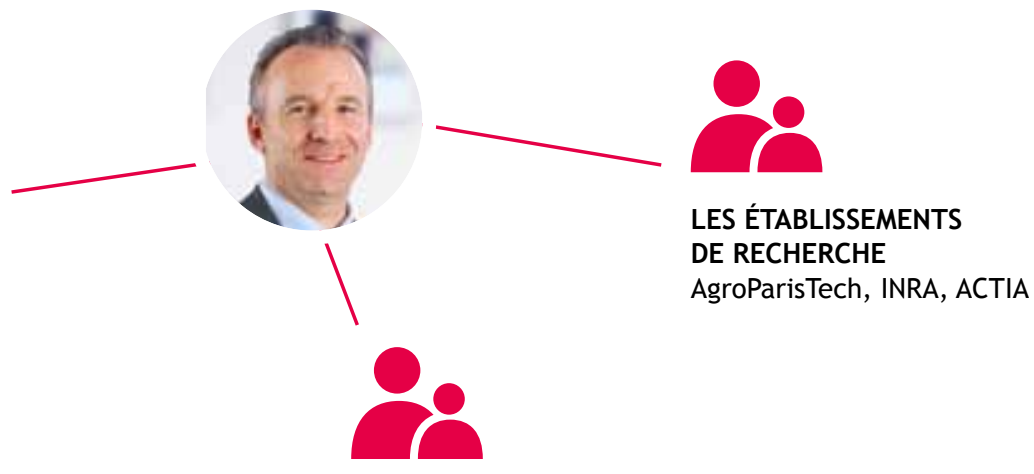

LES ACTEURS PUBLICS

MEIN, MAAF, DGCIS, DGPAAT, CGI,

FranceAgrimer, Bpifrance,

Association des Régions de France,

pôles de compétitivité Vitagora et Valorial

\section{SYNTHĖSE DES ACTIONS DU PLAN}

\begin{tabular}{|c|c|c|c|c|}
\hline Action & Pilote & Exemples de projets & Calendrier & Acteurs publics et leviers d'action \\
\hline $\begin{array}{l}\text { Reconquérir } \\
\text { la compétitivité } \\
\text { des métiers } \\
\text { de la viande }\end{array}$ & $\begin{array}{l}\text { Coop de } \\
\text { France, ACTIA }\end{array}$ & $\begin{array}{l}\text { - Développement d'un automate } \\
\text { de découpe adapté au canard } \\
\text { pour intégrer des systèmes de } \\
\text { reconnaissance } 3 \text { D } \\
\text { - Mécanisation et robotisation du } \\
\text { pliage et de la dépose des cuirs de } \\
\text { bovins sur palette }\end{array}$ & 2014-2017 & $\begin{array}{l}\text { Mobilisation de financements: fonds d'investissement } \\
\text { Robot Start PME, prêts robotiques de Bpifrance, fonds } \\
\text { FEADER et FEDER } \\
\text { Plan Abattoirs } 2014 \text { de FranceAgrimer }\end{array}$ \\
\hline $\begin{array}{l}\text { S'imposer dans } \\
\text { les emballages } \\
\text { et les } \\
\text { conditionnements } \\
\text { actifs, intelligents } \\
\text { et éco-conçus }\end{array}$ & $\begin{array}{l}\text { ANIA, Coop } \\
\text { de France, } \\
\text { ACTIA }\end{array}$ & $\begin{array}{l}\text { - Développement de nouveaux } \\
\text { emballages pour des produits } \\
\text { fragiles, avec intégration d'une puce } \\
\text { permettant leur géolocalisation } \\
\text { - Conception d'emballages à partir } \\
\text { de nouvelles matières premières } \\
\text { végétales, de déchets de production } \\
\text { des IAA etc. }\end{array}$ & 2014-2017 & $\begin{array}{l}\text { Action Démonstrateurs et plateformes technologiques en } \\
\text { énergies renouvelables et décarbonées et chimie verte } \\
\text { de l'ADEME } \\
\text { Mobilisation de financements: Bpifrance, FEADER, FEDER }\end{array}$ \\
\hline $\begin{array}{l}\text { Prendre le } \\
\text { leadership } \\
\text { du froid durable }\end{array}$ & $\begin{array}{l}\text { ANIA, Coop de } \\
\text { France, ACTIA }\end{array}$ & $\begin{array}{l}\text { - Développement de techniques } \\
\text { de production de froid sans utilisation } \\
\text { de gaz réfrigérant } \\
\text { - Gestion des appels des machines } \\
\text { de froid connectées au réseau de } \\
\text { distribution de l'énergie de façon } \\
\text { à pouvoir gérer leur charge. }\end{array}$ & 2014-2017 & $\begin{array}{l}\text { Action Démonstrateurs et plateformes technologiques en } \\
\text { énergies renouvelables et décarbonées et chimie verte } \\
\text { de l'ADEME } \\
\text { Mobilisation de financements Bpifrance: aides à } \\
\text { l'innovation, prêts innovation, prêts verts (ces derniers } \\
\text { avec des critères élargis } \\
\text { au développement et aux investissements pour de } \\
\text { nouvelles techniques et installations frigorifiques } \\
\text { plus économes en énergie, à faible impact } \\
\text { environnemental) ; mobilisation de fonds FEADER, FEDER }\end{array}$ \\
\hline $\begin{array}{l}\text { Garantir la qualité } \\
\text { et la sécurité } \\
\text { des aliments et } \\
\text { des boissons par } \\
\text { des technologies } \\
\text { de contrôle plus } \\
\text { précises et plus } \\
\text { rapides }\end{array}$ & $\begin{array}{l}\text { ANIA, Coop de } \\
\text { France, ACTIA }\end{array}$ & $\begin{array}{l}\text { - Mise au point d'un procédé PCR } \\
\text { (Polymerase chain Reaction) en temps } \\
\text { réel pour la détection de bactéries } \\
\text { pathogènes ou indicatrices d'hygiène } \\
\text { - Mise au point de tests rapides pour la } \\
\text { détection de mycotoxines dans des lots } \\
\text { de céréales } \\
\text { - Développement de capteurs pour } \\
\text { détecter des traces de résidus dans les } \\
\text { opérations de nettoyage en place en } \\
\text { cours de process }\end{array}$ & 2014-2017 & $\begin{array}{l}\text { Mobilisation de financements Bpifrance: aides à } \\
\text { l'innovation, prêts innovation, prêts robotique, prêts } \\
\text { verts, prêts numériques (pour les capteurs de contrôle } \\
\text { en ligne) } \\
\text { Mobilisation de financements FEADER, FEDER }\end{array}$ \\
\hline $\begin{array}{l}\text { Ouvrir le marché } \\
\text { de l'alimentation } \\
\text { fonctionnelle/sur } \\
\text { mesure }\end{array}$ & $\begin{array}{l}\text { ANIA, Coop de } \\
\text { France }\end{array}$ & $\begin{array}{l}\text { Production d'ingrédients, additifs etc. } \\
\text { à partir de techniques d'extraction } \\
\text { naturelles (sans utilisation de solvant } \\
\text { chimique) } \\
\text { Production d'ingrédients, additifs etc. } \\
\text { à partir de co-produits de fabrication } \\
\text { de produits animaux }\end{array}$ & $\begin{array}{l}\text { Lancement } \\
\text { d'un appel } \\
\text { à projets : } \\
\text { juillet } 2014\end{array}$ & $\begin{array}{l}\text { Mobilisation de financements Bpifrance, FEDER, FEADER. } \\
\text { Appel à projet spécifique }(20 \mathrm{M} € \text { ) avec un dépôt au fil de } \\
\text { l'eau, sous forme d'avance remboursable, de subvention } \\
\text { ou d'intervention en fonds propres }\end{array}$ \\
\hline
\end{tabular}




\section{PRODUITS INNOVANTS POUR UNE ALIMENTATION SÛRE, SAINE ET DURABLE}
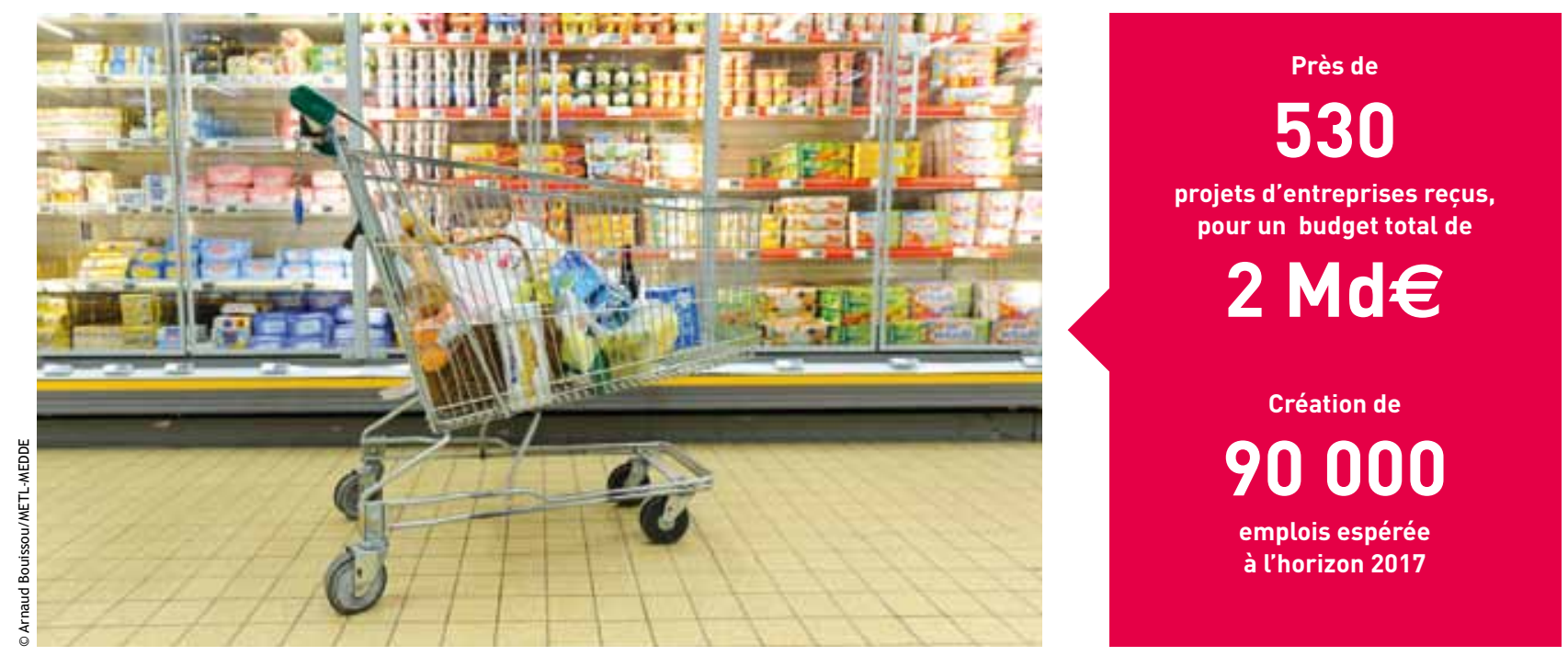

Linéaire de produits alimentaires

- Nous construisons la France de l'alimentation de qualité. Dans la mondialisation et face à la crise de 2008, l'agro-alimentaire est l'un des seuls secteurs de notre industrie à avoir résisté au phénomène de désindustrialisation. Il constitue un soutien précieux à la balance commerciale en y contribuant à hauteur de 8,5 Md€ en 2013. Mais il est parallèlement confronté à des défis de taille, aussi bien sociétaux qu'économiques, sanitaires et environnementaux. Les modes de consommation se sophistiquent, avec une orientation de l'alimentation vers la santé et le bien-être. Les citoyens sont plus vigilants vis-à-vis de la qualité et la traçabilité de ce qu'ils mangent et de l'impact environnemental de leur consommation. - Dans ce monde en pleine évolution, les industries agroalimentaires françaises peuvent reconquérir des parts de marché et contribuer par la qualité de leur offre au bien-être des consommateurs. - Le Plan industriel agro-alimentaire s'inscrit dans cette mission, au carrefour des nouvelles exigences sanitaires, environnementales et sociétales. Il a pour objectif de conforter la position de leader mondial de la France dans le secteur en choisissant de développer des avantages compétitifs qualitatifs, en favorisant l'émergence de produits et de procédés innovants. Partant du constat que le secteur agro-alimentaire réunit des métiers très différents, le groupe projet a choisi de sélectionner des défis transversaux porteurs et pertinents pour la majorité : reconquérir la compétitivité des métiers de la viande, s'imposer dans les emballages du futur, prendre le leadership du froid durable, garantir la qualité et la sécurité des aliments et boissons et ouvrir le marché de l'alimentation fonctionnelle, au croisement du bien-être et de la nutrition. 


\section{ÉQUIPE DU PLAN}

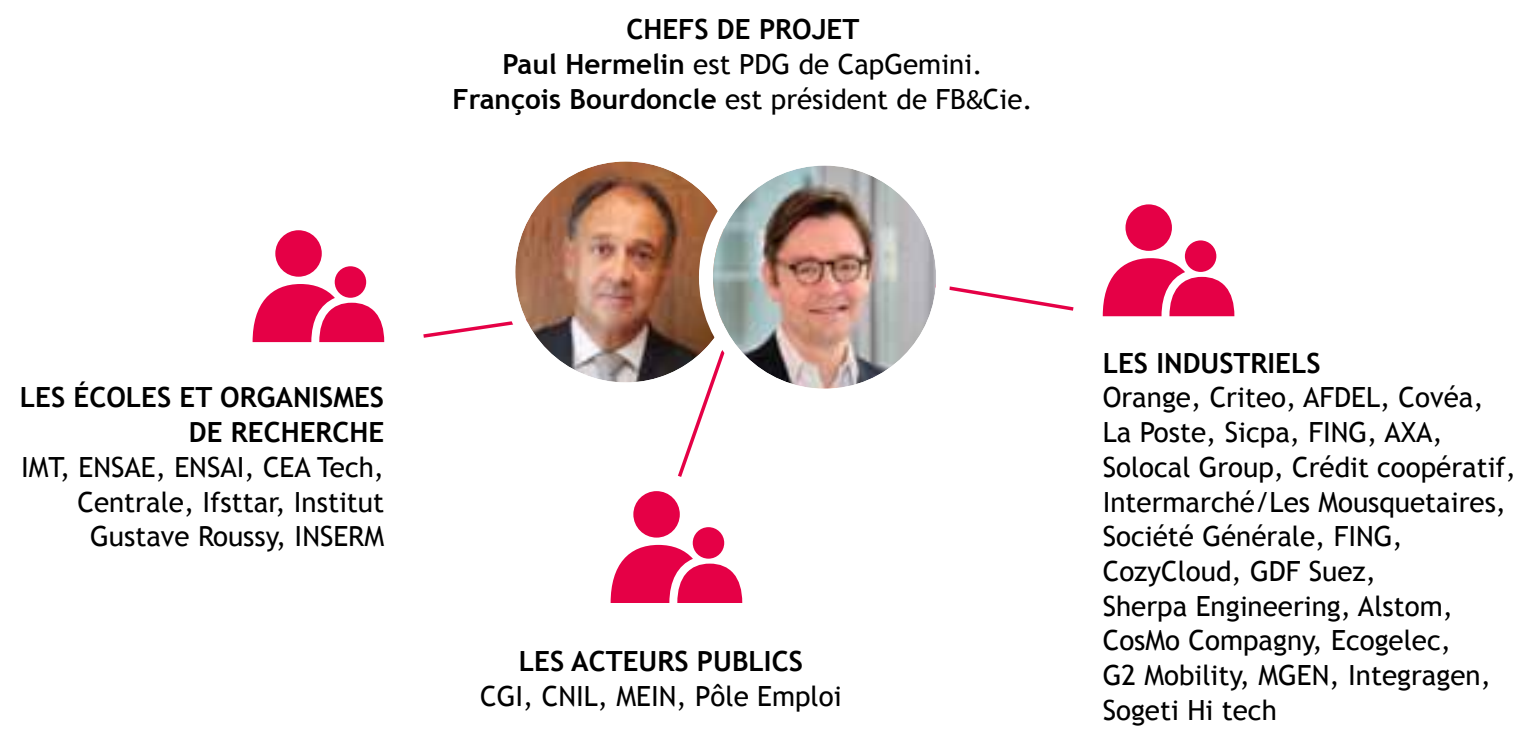

\section{SYNTHĖSE DES ACTIONS DU PLAN}

\begin{tabular}{|c|c|c|}
\hline Action & Calendrier & Finalité/livrables \\
\hline \multicolumn{3}{|l|}{ Développement de l'écosystème «Big Data» en France } \\
\hline Formation de «data scientists" & Second semestre 2014 & $\begin{array}{l}\text { Mise en place de cursus de formation adaptés aux } \\
\text { besoins du Big Data: MOOC, masters... }\end{array}$ \\
\hline Accès des startups aux données et aux infrastructures & Second semestre 2014 & $\begin{array}{l}\text { Création d'un centre qui sera un lieu privilégié } \\
\text { pour les acteurs privés ou publics, détenteurs de } \\
\text { jeux de données, souhaitant travailler avec des } \\
\text { startups dans un modèle « d'innovation ouverte » }\end{array}$ \\
\hline Soutien au financement et à l'accélération des startups & Courant 2014 & $\begin{array}{l}\text { Financement pour les startups et les PME dans } \\
\text { le cadre du Concours Mondial d'Innovation } \\
\text { Soutien aux éventuels projets d'accélérateurs } \\
\text { Big Data et aux fonds d'amorçage qui pourraient } \\
\text { émerger dans le cadre de l'initiative French Tech }\end{array}$ \\
\hline Observatoire des usages & Fin 2014 - début 2015 & $\begin{array}{l}\text { Lancement d'une étude sur les enjeux } \\
\text { économiques liés au Big Data dans les filières } \\
\text { utilisatrices }\end{array}$ \\
\hline \multicolumn{3}{|l|}{ Lancement d'initiatives sectorielles sur le Big Data } \\
\hline \multirow[t]{2}{*}{$\begin{array}{l}\text { Favoriser la diffusion des technologies du Big Data } \\
\text { dans le secteur privé }\end{array}$} & Premières initiatives lancées en 2014 & $\begin{array}{l}\text { Soutien à des projets visant à favoriser la } \\
\text { diffusion du Big Data dans diverses filières } \\
\text { industrielles. }\end{array}$ \\
\hline & & $\begin{array}{l}\text { Des initiatives seront lancées au second semestre } \\
\text { dans le secteur de l'énergie, des assurances, } \\
\text { de la traçabilité... }\end{array}$ \\
\hline Moderniser l'action publique grâce à l'utilisation du Big Data & Premières initiatives lancées en 2014 & $\begin{array}{l}\text { Mise en place de projets innovants dans le } \\
\text { domaine de l'action publique grâce au Big Data : } \\
\text { emploi, santé... }\end{array}$ \\
\hline \multicolumn{3}{|l|}{ Évolutions de la réglementation } \\
\hline $\begin{array}{l}\text { Adapter le contexte réglementaire et législatif } \\
\text { pour permettre le développement d'une filière Big Data }\end{array}$ & Second semestre 2014 & $\begin{array}{l}\text { Permettre des certifications de processus } \\
\text { industriels afin de favoriser l'utilisation } \\
\text { du Big Data }\end{array}$ \\
\hline & & $\begin{array}{l}\text { Etudier l'opportunité d'adapter le contexte } \\
\text { réglementaire et législatif }\end{array}$ \\
\hline
\end{tabular}




\section{BIG DATA}
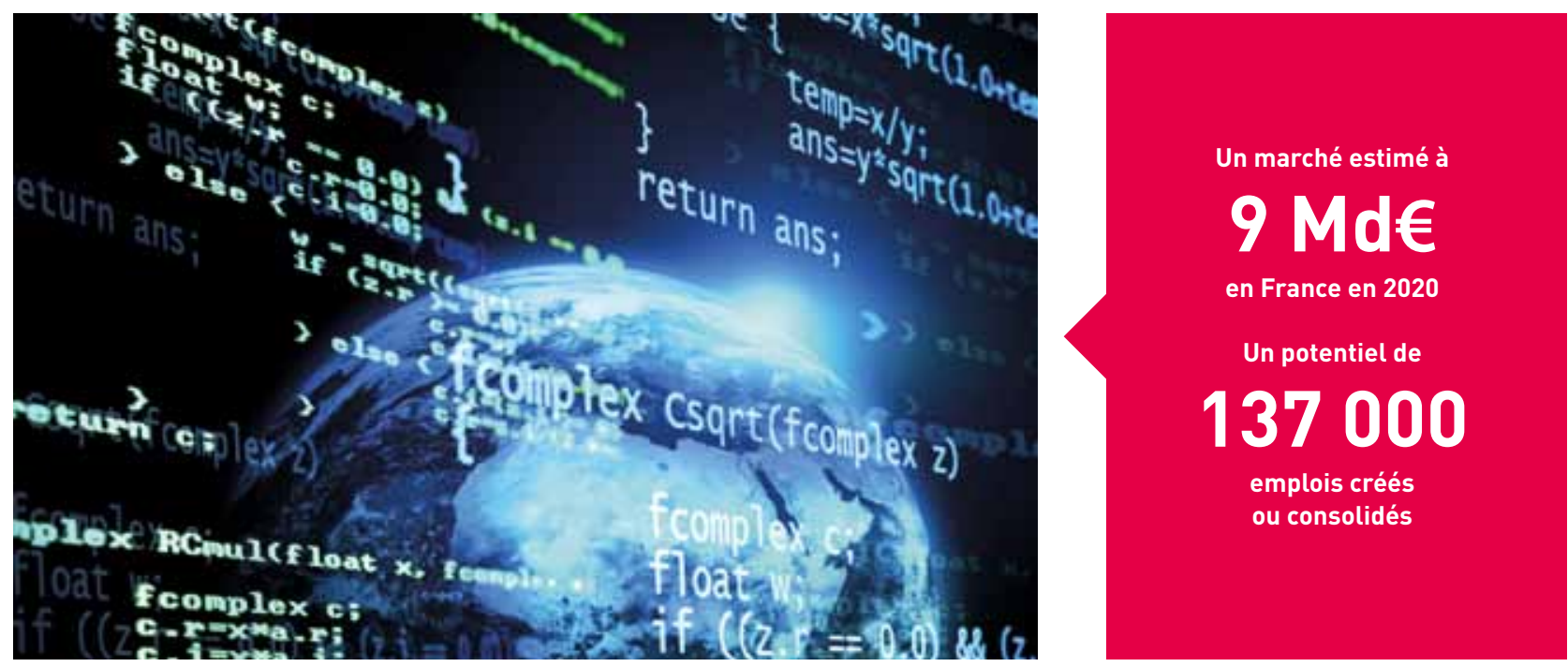

Données informatiques, lignes de code

- Nous construisons la France de la souveraineté numérique. - Le développement des services de l'internet, des ordinateurs, smartphones, tablettes et objets connectés engendre la production, le traitement et l'utilisation de quantités inédites de données numériques. Ces données représentent, dans tous les secteurs, une source de valeur nouvelle et encore trop peu exploitée. - Les enjeux liés au Big Data sont considérables, tant sur le plan économique que pour garantir un espace numérique protecteur de la vie privée et des libertés fondamentales. - Le plan vise à positionner la France comme le pays de référence dans le domaine du Big Data en associant à la fois des fournisseurs de technologies - essentiellement des PME et des startups -, de grands groupes utilisateurs, mais également des instituts d'enseignement supérieur. \ Le plan favorisera le développement d'un écosystème industriel compétitif dans le domaine du Big Data. Pour ce faire, il facilitera par exemple l'accès des startups aux données de grandes entités publiques et privées, ainsi qu'aux infrastructures nécessaires pour exploiter ces données. L'accent sera également mis sur la formation de « data scientists ». - Le plan permettra en outre le lancement d'initiatives visant à accélérer la diffusion du Big Data dans divers secteurs utilisateurs: tourisme, transports, énergie, assurance... Le plan prévoit également des actions de modernisation de l'action publique grâce au Big Data, par exemple dans le domaine de l'emploi. - Il s'attachera enfin à adapter le contexte réglementaire et législatif à l'émergence des traitements de données massifs, dans un souci conjoint d'innovation et de respect de la vie privée de chacun. - Les actions du plan représentent un potentiel de création ou de consolidation de plus de 137000 emplois d'ici 2020. Elles invitent les acteurs de notre pays à s'organiser pour que la puissance publique comme les entreprises puissent pleinement tirer profit du Big Data. 


\title{
ÉQUIPE DU PLAN
}

\author{
CHEFS DE PROJET
}

Thierry Breton est PDG d'ATOS.

Octave Klaba est fondateur et directeur général d’OVH.

LES ÉCOLES ET ORGANISMES DE RECHERCHE INRIA pour le MENESR

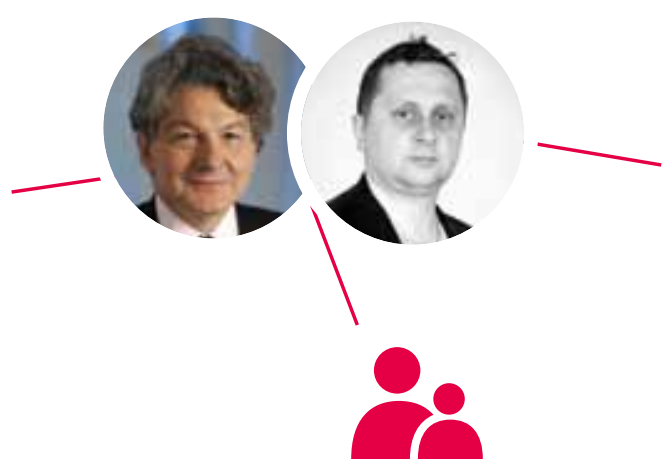

LES ACTEURS PUBLICS

Bpifrance, CGI, MEIN, DGCIS

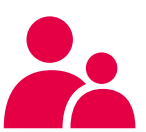

LES INDUSTRIELS

Atos, OVH, Bull, Orange,

Numergy, Cloudwatt, Axway, Cegid, Talentsoft, Prestashop,

Cozy cloud, Jolicloud

\section{SYNTHĖSE DES ACTIONS DU PLAN}

\begin{tabular}{|c|c|c|}
\hline Action & Calendrier & Finalité/livrables \\
\hline \multicolumn{3}{|l|}{ Mesures en faveur de la demande } \\
\hline $\begin{array}{l}\text { Création d'un label sécurité } \\
\text { «Secure cloud» pour les offres } \\
\text { de cloud computing }\end{array}$ & $\begin{array}{l}\text { Premières expérimentations } \\
\text { au second semestre } 2014\end{array}$ & $\begin{array}{l}\text { Labellisation à vocation européenne pour les acteurs hébergeant } \\
\text { et traitant les données sur le territoire européen selon des normes } \\
\text { contractuelles de qualité et de sécurité élevées }\end{array}$ \\
\hline $\begin{array}{l}\text { Exemplarité de l'action publique } \\
\text { et création d'une place de marché } \\
\text { numérique pour les collectivités locales }\end{array}$ & $\begin{array}{l}\text { Courant 2014, } \\
\text { généralisation en } 2015\end{array}$ & $\begin{array}{l}\text { Passage au cloud de l'informatique de l'État, des organismes publics } \\
\text { et des collectivités locales et création d'un guide de bonnes } \\
\text { pratiques; création de « places de marché cloud» proposant des } \\
\text { applications validées et sécurisées pour les collectivités locales } \\
\text { (puis, plus largement, les entités publiques) }\end{array}$ \\
\hline Neutralité cloud dans les marchés publics & $\begin{array}{l}\text { Second semestre } 2014 \text { (premiers } \\
\text { marchés), } 2015 \text { (généralisation) }\end{array}$ & $\begin{array}{l}\text { Mise en place de procédures de marché public assurant une neutralité } \\
\text { vis-à-vis des offres cloud par rapport aux solutions internalisées }\end{array}$ \\
\hline $\begin{array}{l}\text { Adoption du cloud par les entreprises } \\
\text { (aide à la transformation numérique) }\end{array}$ & Courant 2014 & $\begin{array}{l}\text { Amplifier l'accompagnement des entreprises, et notamment des PME, } \\
\text { vers le cloud et la création ou l'extension de relations numériques } \\
\text { avec leurs clients; promotion/création de places de marché } \\
\text { numériques de solutions pour PME }\end{array}$ \\
\hline $\begin{array}{l}\text { Adoption du cloud par le grand public } \\
\text { («cloud personnel») }\end{array}$ & Courant 2014 & $\begin{array}{l}\text { Lancement d'une initiative autour des domaines personnels pour } \\
\text { permettre à chaque Français de disposer de son nom de domaine } \\
\text { à faible coût, de la gestion d'identité numérique, des espaces de } \\
\text { stockage personnels sécurisés... }\end{array}$ \\
\hline \multicolumn{3}{|l|}{ Mesures en faveur de l'offre } \\
\hline $\begin{array}{l}\text { Garantir un traitement sécurisé } \\
\text { des données en Europe (normes élevées } \\
\text { de qualité et de sécurité) }\end{array}$ & $\begin{array}{l}2015 \text { (à coordonner avec la } \\
\text { Commission Européenne) }\end{array}$ & $\begin{array}{l}\text { Mise en place de règles harmonisées de qualité de service } \\
\text { et de protection des données au niveau européen }\end{array}$ \\
\hline $\begin{array}{l}\text { Attractivité de la France pour } \\
\text { l'installation de datacenters }\end{array}$ & Second semestre 2014 & Simplification des démarches de création de datacenters \\
\hline $\begin{array}{l}\text { Aide aux éditeurs de logiciels à la } \\
\text { transition vers le cloud }\end{array}$ & Second semestre 2014 & $\begin{array}{l}\text { Création d'une «plate-forme Tremplin» globale permettant } \\
\text { la transformation cloud des offres des acteurs de l'informatique } \\
\text { et notamment des éditeurs de logiciels }\end{array}$ \\
\hline $\begin{array}{l}\text { Soutien à l'innovation dans le cloud, avec } \\
\text { une orientation usage }\end{array}$ & Courant 2014 & $\begin{array}{l}\text { Soutien à des projets visant à développer des services cloud } \\
\text { correspondant aux besoins de communautés d'utilisateurs (éducation, } \\
\text { santé, seniors, etc.) }\end{array}$ \\
\hline $\begin{array}{l}\text { Renforcer les formations cloud, } \\
\text { l'attractivité et l'organisation de la filière }\end{array}$ & Courant 2014 & $\begin{array}{l}\text { Développement de cursus en formation initiale et continue, s'appuyant } \\
\text { sur des méthodes pédagogiques issues du numérique. Contribution à } \\
\text { l'attractivité des métiers, et création d'un " observatoire du cloud " }\end{array}$ \\
\hline
\end{tabular}




\section{CLOUD COMPUTING}
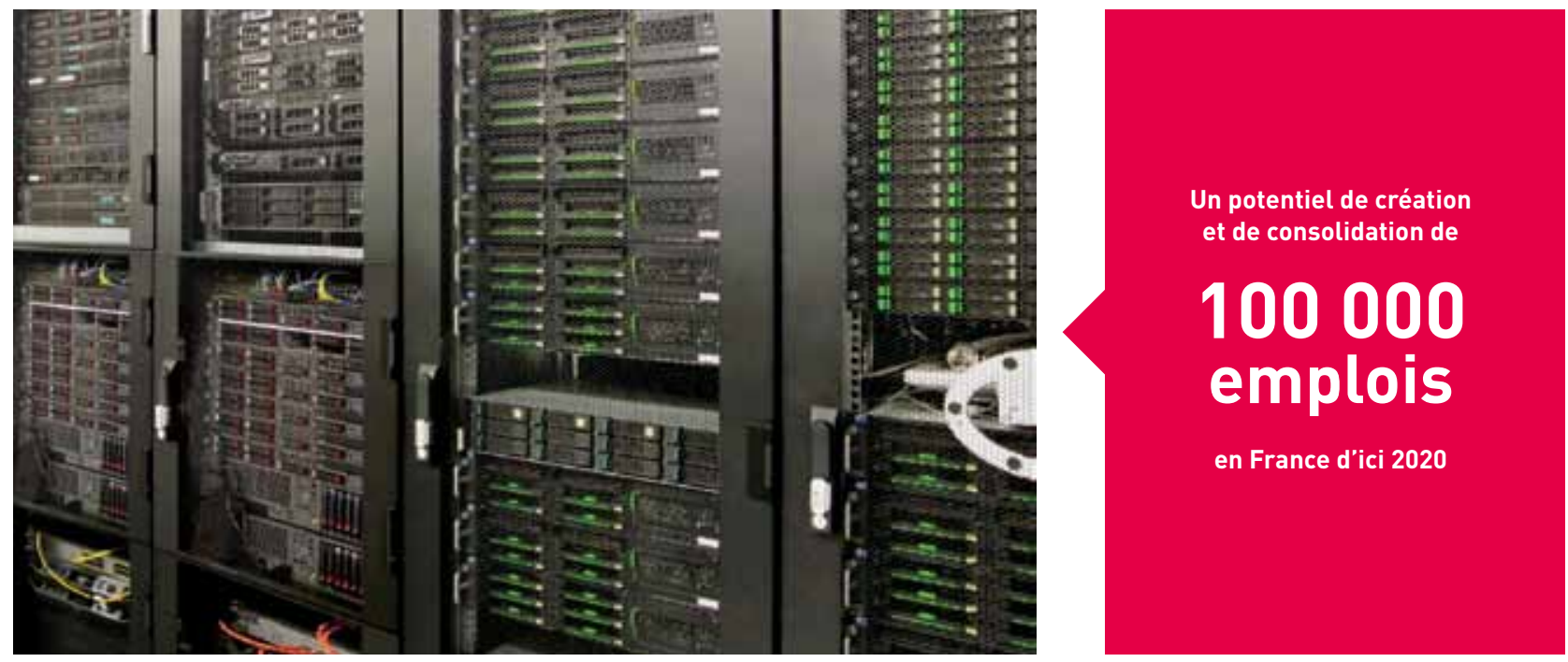

Serveur informatique

Nous construisons la France de l'accès et du partage sécurisés et simplifiés des données informatiques, en développant un puissant écosystème industriel. - Facilitant un accès «mobile» aux services numériques, en tous lieux et indépendamment de l'appareil utilisé, le cloud computing permet d'accroître « à la demande » l'efficacité du stockage et du traitement de l'information, tout en réduisant significativement les dépenses des consommateurs de ressources informatiques. Les utilisateurs paient uniquement pour les ressources informatiques qu'ils utilisent effectivement. Simultanément, les opérateurs du cloud computing sont confrontés à un changement de paradigme majeur, car ils doivent, à la place du client, assumer des investissements de plus en plus importants. - La révolution technologique du cloud computing, qui est à la base d'autres révolutions comme le Big Data, représente une fantastique opportunité en termes de croissance, de productivité, d'emploi et de création de nouveaux services innovants. L'économie du cloud computing enregistre actuellement des taux de croissance de $20 \%$ à $30 \%$ par an dans le monde, avec un marché représentant déjà un chiffre d'affaires de plus de 2 milliards d'euros en France en 2012. - Le plan cloud computing vise en premier lieu à accroître la confiance envers ses solutions, grâce notamment à la création d'un label « Secure cloud » et à la promotion d'une réglementation européenne plus protectrice. Ce plan encourage le développement d'une filière du cloud français et européen pour renforcer notre souveraineté numérique sur les données personnelles, tout en dynamisant la compétitivité de nos entreprises. - Les actions du plan représentent un potentiel de création ou de consolidation de plus de 100000 emplois d'ici 2020. Elles invitent les acteurs de notre pays à s'organiser pour que la puissance publique comme les entreprises et les particuliers puissent pleinement tirer profit du cloud dans un contexte approprié de qualité, de protection des données et de sécurité. 


\section{ÉQUIPE DU PLAN}

CHEFS DE PROJET

Jean-Yves Hepp est le président et fondateur de Unowhy. Deborah Elalouf est présidente de Tralalère.

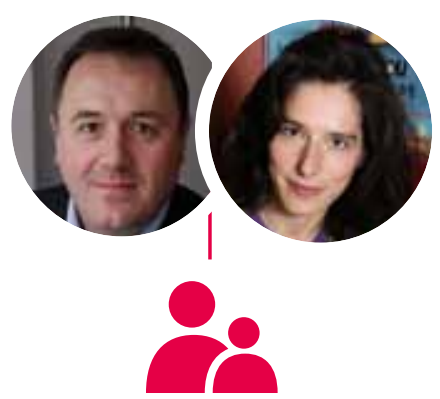

$>$ Les acteurs de la filière, éditeurs traditionnels, producteurs de ressources éducatives numériques, fabricants de terminaux, fournisseurs de services et d'infrastructures, opérateurs de plateformes logicielles, ont été auditionnés par les chefs de projet.

$>$ Les collectivités locales, les enseignants et les équipes pédagogiques seront pleinement associés à la mise en œuvre du plan.

\section{SYNTHÈSE DES ACTIONS DU PLAN}

\begin{tabular}{|c|c|c|c|c|}
\hline Action & Pilote & Calendrier & $\begin{array}{l}\text { Acteurs publics et leviers } \\
\text { d'action, acteurs privés }\end{array}$ & Finalités/livrables \\
\hline \multirow{2}{*}{$\begin{array}{l}\text { Soutien à une politique } \\
\text { massive d'équipement } \\
\text { des élèves et des } \\
\text { enseignants du premier } \\
\text { degré et du second }\end{array}$} & \multirow[t]{2}{*}{$\begin{array}{l}\text { MENESR, } \\
\text { MEIN }\end{array}$} & 2020 & $\begin{array}{l}\text { MENESR, MEIN, collectivités } \\
\text { territoriales }\end{array}$ & $\begin{array}{l}70 \% \text { des élèves de primaire et collège équipés en } \\
\text { terminaux individuels et collectifs à l'horizon } 2020\end{array}$ \\
\hline & & Fin 2014 & Commande publique & $\begin{array}{l}\text { Lancement des premiers appels d'offre lauréats par les } \\
\text { collectivités territoriales dès la fin } 2014\end{array}$ \\
\hline \multirow{2}{*}{$\begin{array}{l}\text { Développement du } \\
\text { marché des ressources } \\
\text { numériques éducatives } \\
\text { pour guider la transition } \\
\text { en faveur du numérique }\end{array}$} & \multirow[t]{2}{*}{$\begin{array}{l}\text { MENESR, } \\
\text { MEIN }\end{array}$} & 2020 & $\begin{array}{l}\text { MENESR, MEIN, collectivités } \\
\text { territoriales }\end{array}$ & $\begin{array}{l}\text { Part de plus de } 60 \% \text { de ressources numériques dans les } \\
\text { budgets dédiés aux contenus pédagogiques }\end{array}$ \\
\hline & & Fin 2014 & Commande publique & $\begin{array}{l}\text { Lancement des premiers achats de ressources numériques } \\
\text { éducatives }\end{array}$ \\
\hline \multirow{2}{*}{$\begin{array}{l}\text { Création d'offres } \\
\text { de Cloud spécifiques } \\
\text { à l'éducation en } \\
\text { s'appuyant sur le label } \\
\text { "Secure Cloud» } \\
\text { du plan industriel Cloud }\end{array}$} & \multirow[t]{2}{*}{ MEIN } & \multirow[t]{2}{*}{$\begin{array}{l}1 \text { er semestre } \\
2015\end{array}$} & \multirow[t]{2}{*}{$\begin{array}{l}\text { MEIN, MENESR, CNIL, collectivités } \\
\text { territoriales, fournisseurs de } \\
\text { solutions Cloud } \\
\text { et opérateurs télécom }\end{array}$} & $\begin{array}{l}\text { Attribution des premiers labels Cloud éducatif garantissant } \\
\text { la localisation et la confidentialité des données des élèves } \\
\text { dans le respect de la réglementation }\end{array}$ \\
\hline & & & & Solutions cloud labellisées \\
\hline $\begin{array}{l}\text { Soutien au financement } \\
\text { de l'innovation et à } \\
\text { l'accompagnement } \\
\text { des entreprises dans } \\
\text { le déploiement du } \\
\text { numérique à l'école }\end{array}$ & $\begin{array}{l}\text { MEIN, } \\
\text { MENESR }\end{array}$ & Fin 2014 & $\begin{array}{l}\text { Sec. Comm. Ext, MAE, UbiFrance, } \\
\text { MJS, et MCC le cas échéant, } \\
\text { BPIfrance, CGI, MESR, ANR }\end{array}$ & $\begin{array}{l}\text { Ouverture des fonds aux candidatures des entreprises pour } \\
\text { la création de solutions numériques éducatives innovantes } \\
\text { et prêtes à être déployées, en France et à l'international }\end{array}$ \\
\hline \multirow{2}{*}{$\begin{array}{l}\text { Garantie d'un cadre de } \\
\text { confiance pour l'usage } \\
\text { des ressources et mise } \\
\text { en place des conditions } \\
\text { d'un choix éclairé } \\
\text { pour les prescripteurs } \\
\text { de ressources }\end{array}$} & \multirow[t]{2}{*}{$\begin{array}{l}\text { MENESR, } \\
\text { MEIN }\end{array}$} & 2014-2015 & \multirow{2}{*}{$\begin{array}{l}\text { MENESR, MEIN, collectivités } \\
\text { territoriales, opérateurs et } \\
\text { développeurs de portails et } \\
\text { d'outils d'identification, de mise } \\
\text { en valeur, d'achat et d'accès aux } \\
\text { contenus }\end{array}$} & $\begin{array}{l}\text { Étude sur la concurrence entre édition scolaire publique } \\
\text { et privée } \\
\text { Pilote d'un système d'accès aux ressources }\end{array}$ \\
\hline & & 2016 & & Mise en ligne opérationnelle \\
\hline $\begin{array}{l}\text { Mise en place d'un } \\
\text { cadre de référence } \\
\text { pour accompagner } \\
\text { l'achat public dans le } \\
\text { numérique éducatif }\end{array}$ & MENESR & $\begin{array}{l}\text { Septembre } \\
2014\end{array}$ & $\begin{array}{l}\text { MENESR, MEIN, collectivités } \\
\text { territoriales }\end{array}$ & $\begin{array}{l}\text { Publication en ligne d'un cadre de référence des achats } \\
\text { numériques éducatifs }\end{array}$ \\
\hline
\end{tabular}




\section{E-ÉDUCATION}
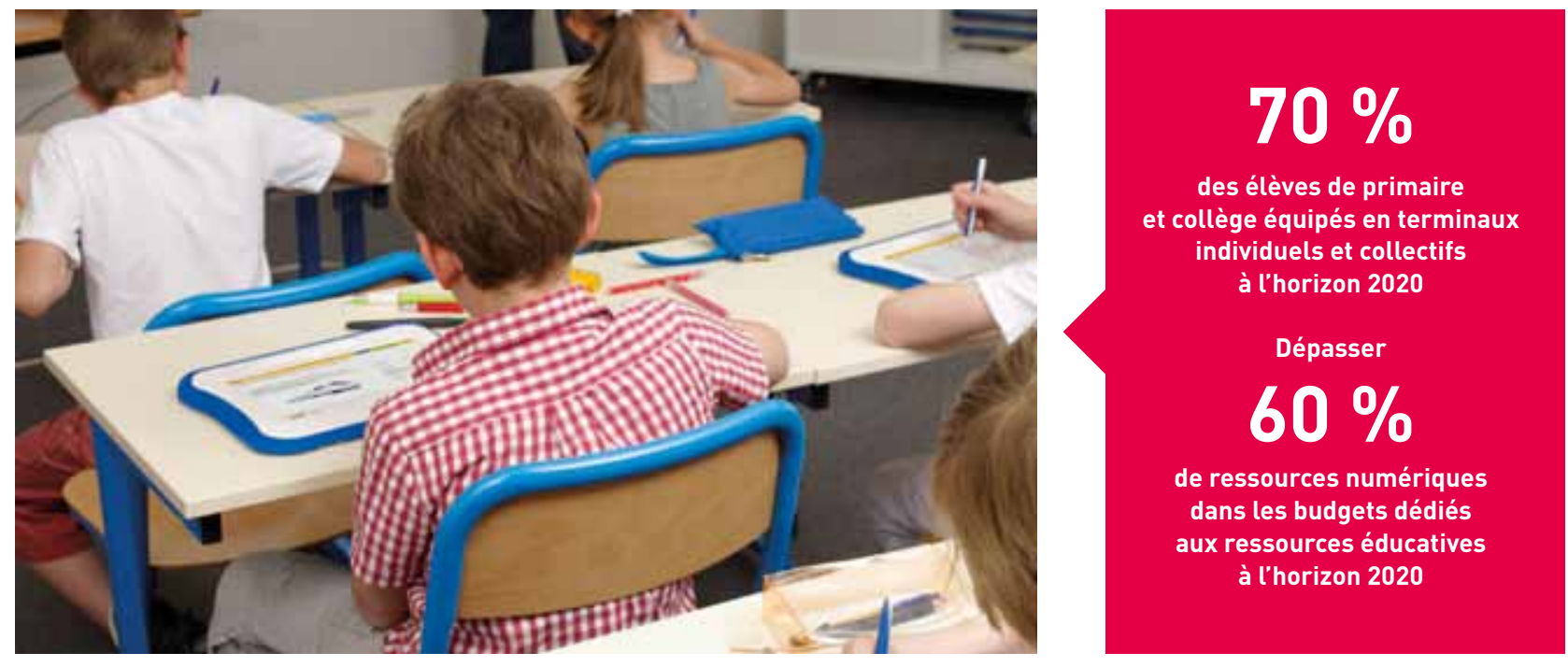

Élèves étudiant sur des tablettes numériques

- Nous construisons la France de la révolution pédagogique numérique pour tous. évoluer toutes les formes traditionnelles de l'éducation: la relation entre l'enseignant ou le formateur et ses élèves, les relations entre les pédagogues, la relation entre les élèves, la relation entre l'Ecole et les familles. L'e-Éducation est une formidable occasion de développer l'interactivité et la créativité de tous.

développement d'une filière industrielle française du numérique éducatif performante est un enjeu politique de souveraineté nationale, une source de création d'emplois qualifiés et un élément clef pour permettre à la France de continuer à faire entrer l'Ecole dans l'ère du numérique : cela constitue donc un investissement essentiel pour l'avenir de la jeunesse. Il existe un tissu dynamique de startups, travaillant sur le développement d'applications et la conception de ressources numériques et d'objets connectés à usage éducatif. Les enjeux économiques sont également considérables: le marché mondial de l'e-Éducation est ainsi évalué à 91 milliards de dollars, avec une perspective de croissance annuelle de 23 \% d'ici à 2017. — La France peut s'appuyer sur de nombreuses expérimentations en milieu éducatif, auxquelles la stratégie numérique pour la refondation de l'École du Gouvernement donne un nouvel élan. - Il n'est pas de stratégie numérique pour la refondation de l'École sans volet industriel. Réduire la fracture numérique éducative, structurer l'offre et la demande en offrant des débouchés concrets aux PME en France et à l'international aux différents niveaux d'enseignement (primaire, secondaire), accompagner la transition numérique des acteurs de l'éducation, faire émerger un écosystème global de l'e-Éducation, depuis les contenus et services jusqu'au matériel, tels sont les enjeux les plus importants de ce plan industriel « e-Éducation ». 


\section{ÉQUIPE DU PLAN}

\section{CHEF DE PROJET}

Philippe Keryer est directeur de la Stratégie et de l'Innovation d'Alcatel-Lucent.

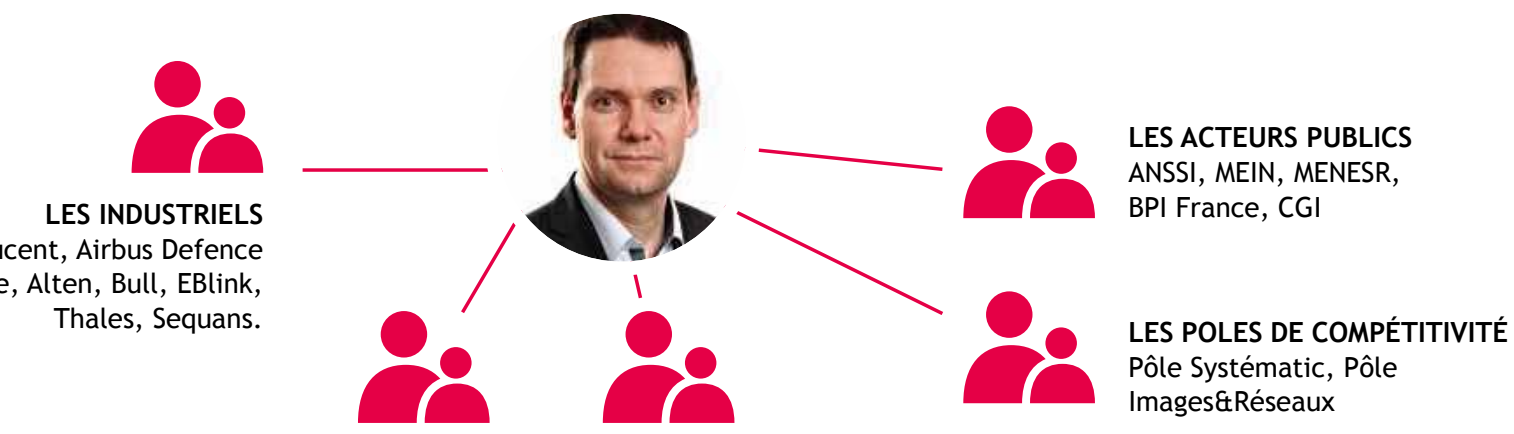

LES OPERATEURS TELECOMS

Bouygues Telecom, Completel Numéricable, Free, Hub One, Orange

\section{LES ÉCOLES ET ORGANISMES \\ DE RECHERCHE}

Inria, IRT SystemX,

IRT B COM, LINCS, NOVEA,

Telecom ParisTech

LES ACTEURS PUBLICS

ANSSI, MEIN, MENESR,

BPI France, CGI

Pôle Systématic, Pôle

Images\&Réseaux

\section{SYNTHĖSE DES ACTIONS DU PLAN}

\begin{tabular}{|c|c|c|c|}
\hline Action & Pilote & Calendrier & Finalités/livrables \\
\hline \multicolumn{4}{|l|}{ Très haut débit mobile } \\
\hline Travaux de recherche sur la $5 \mathrm{G}$ & Orange & 2014-2015 & $\begin{array}{l}\text { Former un consortium pour les appels à projet européen } \\
\text { du } 5 \text { GPPP de novembre } 2014 \text {, en vue d'établir une vision } \\
\text { commune européenne pour la définition de la future } \\
\text { norme } 5 \text { G }\end{array}$ \\
\hline \multicolumn{4}{|l|}{ Très haut débit fixe } \\
\hline $\begin{array}{l}\text { Pôle de référence et réseau de centres } \\
\text { de formation aux métiers de déploiement } \\
\text { de la fibre }\end{array}$ & Novea & Courant 2015 & $\begin{array}{l}\text { Créer un réseau de centres de formation réparti sur } \\
\text { l'ensemble du territoire organisé autour d'un pôle de } \\
\text { référence afin de déployer le plan national de formation } \\
\text { Favoriser et accélérer la formation aux métiers de } \\
\text { déploiement du réseau THD fixe (fibre) }\end{array}$ \\
\hline \multicolumn{4}{|l|}{ Infrastructure sécurisée et virtualisation } \\
\hline $\begin{array}{l}\text { Évolution de la réglementation sur } \\
\text { la sécurisation des réseaux actuels } \\
\text { et virtualisés }\end{array}$ & ANSSI & Second semestre 2014 & $\begin{array}{l}\text { Recommandations concernant l'évolution de la } \\
\text { réglementation sur la sécurité des réseaux }\end{array}$ \\
\hline \multirow[t]{2}{*}{$\begin{array}{l}\text { Démonstrateur expérimental multi-usages } \\
\text { de virtualisation de réseau et plateforme } \\
\text { de référence sécurité }\end{array}$} & Alcatel-Lucent & 2014-2015 & $\begin{array}{l}\text { Plateforme de référence de virtualisation accueillant les } \\
\text { différentes briques constitutives d'une infrastructure } \\
\text { virtualisée et accueillant les fournisseurs d'API et } \\
\text { d'applications }\end{array}$ \\
\hline & ANSSI & $2014-2015$ & $\begin{array}{l}\text { Mise en œuvre d'une plateforme de référence sécurité } \\
\text { permettant la validation des solutions de sécurité réseau }\end{array}$ \\
\hline \multicolumn{4}{|l|}{ Radiocommunications sécurisées } \\
\hline \multirow{2}{*}{$\begin{array}{l}\text { Démonstrateur d'un réseau } 4 \mathrm{G} \\
\text { permettant d'offrir un réseau haut-débit } \\
\text { et sécurisé, dédié aux services de secours } \\
\text { et aux opérateurs d'importance vitales } \\
\text { (OIV) }\end{array}$} & $\begin{array}{l}\text { Airbus Defence } \\
\text { \& Space }\end{array}$ & Second semestre $2014-2015$ & $\begin{array}{l}\text { Projet de R\&D «LTE4PMR»: développement d'une } \\
\text { solution de réseau mobile professionnel PMR } 400 \mathrm{MHz} \text { et } \\
\text { standardisation }\end{array}$ \\
\hline & Thales & Second semestre 2014-2015 & $\begin{array}{l}\text { Démonstrateur LTE PMR } 700 \mathrm{MHz} \text { : basé sur les cas } \\
\text { d'usage des réseaux commerciaux de la feuille de route } \\
\text { « communications sécurisées haut débit » ACN }\end{array}$ \\
\hline \multicolumn{4}{|c|}{ Développement économique des start-ups et PME } \\
\hline Accompagnement des start-ups et PME & EBlink & $\begin{array}{l}\text { Tout au long des projets } \\
\text { lancés }\end{array}$ & $\begin{array}{l}\text { Recréer un écosystème, promouvoir la participation de } \\
\text { start-ups et de PME innovantes qui ont un potentiel de } \\
\text { croissance à partir de la France }\end{array}$ \\
\hline
\end{tabular}




\section{SOUVERAINETÉ TÉLÉCOMS}

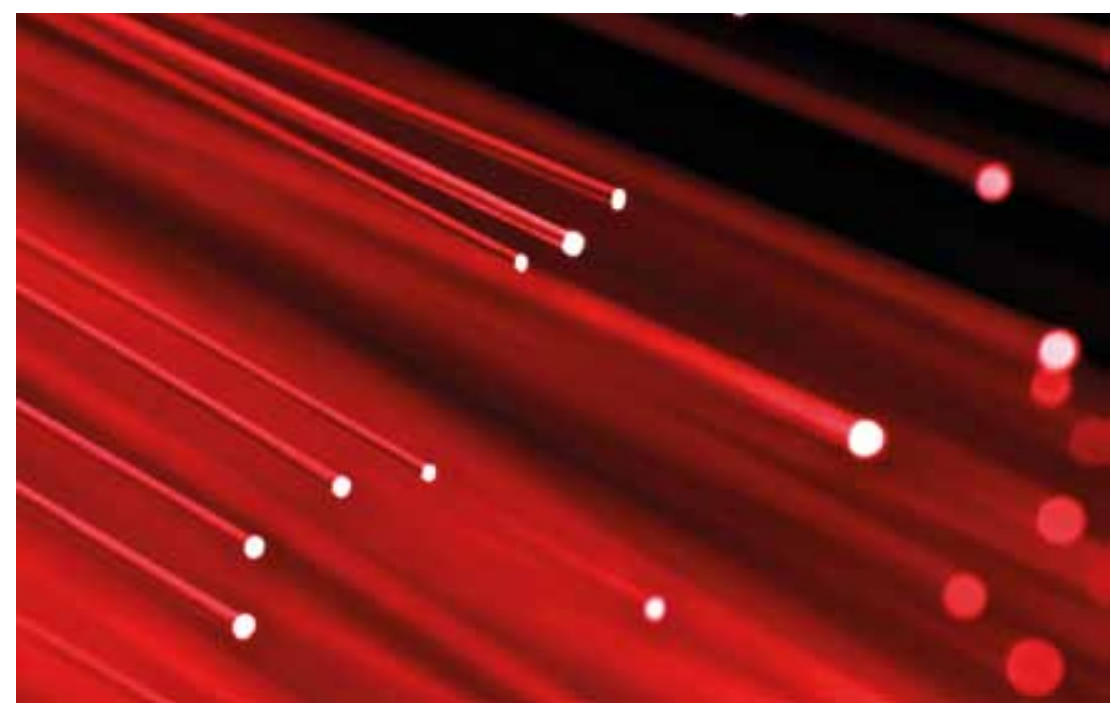

\section{$5,5 \%$}

du PIB en 2015, c'est le poids du numérique en France

La $5 G$, ce sont des capacités

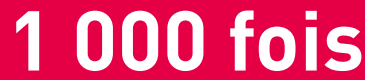

supérieures à celles des réseaux mobiles actuels

\section{0 milliards}

d'objets connectés dans le monde en 2020

Fibres optiques

- Nous construisons la France qui met la défense de sa souveraineté numérique au service de la compétitivité de ses entreprises - Le numérique occupe une place croissante dans notre vie quotidienne et professionnelle. En 2012, le marché combiné des matériels, logiciels, services en ligne et télécoms représentait 2450 milliards d'euros, et des investissements d'environ 8 milliards d'euros pour la France. - L'industrie française du numérique n'en est pourtant qu'à une première phase de son développement et est confrontée à des enjeux déterminants pour son futur. - Le développement d'un écosystème télécom national devient crucial face à la globalisation des échanges de données et à la numérisation de nombreux usages critiques. - Il est nécessaire d'assurer la compétitivité de l'industrie française, mais aussi une maîtrise nationale des infrastructures et de leur sécurisation. - Les réseaux doivent devenir plus rapides (très haut débit), plus sécurisés, fournissant des services plus intégrés avec notamment le «Cloud » et toujours plus proches des utilisateurs. - Pour cela, la France possède un savoir-faire important qui s'appuie sur l'innovation technologique de nos multinationales et de nos PME et start-ups ainsi que sur l'excellence de nos filières académiques. Ce savoir-faire sera mobilisé pour développer des réseaux de communication haut débit et sécurisés, destinés aux opérateurs d'importance vitale (technologie LTE/4G), pour préparer l'ultra-haut débit mobile (5G) et pour apporter des solutions de supervision et de sécurisation de nos infrastructures. Ce plan fait des propositions concrètes de recherche, de développement et d'industrialisation, et des recommandations pour les évolutions réglementaires ou législatives et pour la formation aux nouveaux métiers. \ Nous préparons ainsi nos industries à l'évolution des besoins et formons un socle pour la conquête de parts de marché à l'international. 


\section{ÉQUIPE DU PLAN}

CHEF DE PROJET

Laurent Malier est directeur du CEA-LETI.

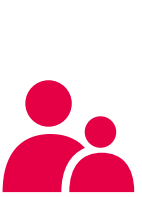

LES INDUSTRIELS

STMicroelectronics, Altis, Dolphin Integration, IPDIA, EADS, Syndicats professionnels ACSIEL et GIFAS, et plus de 40 industriels

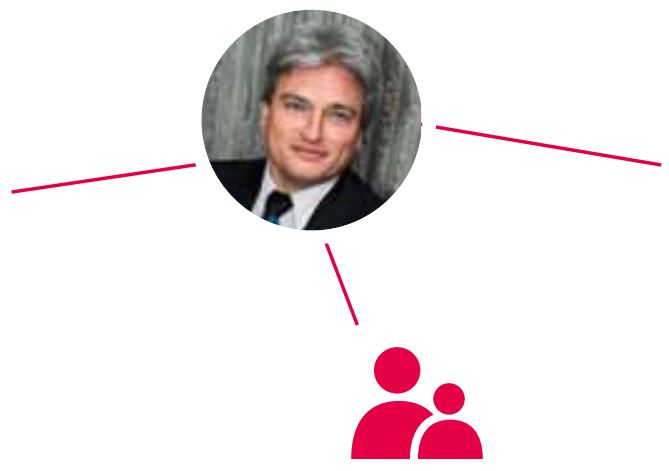

LES ACTEURS PUBLICS MEIN, DGA, CGI

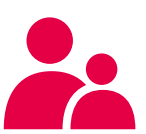

LES ÉTABLISSEMENTS DE RECHERCHE

plus de 30 laboratoires issus de l'université, du CNRS et du CEA

\section{SYNTHĖSE DES ACTIONS DU PLAN}

Action Pilote Calendrier Acteurs Finalités/livrables

Assurer un positionnement de leader européen dans les technologies de production de composants nanoélectroniques

\begin{tabular}{|c|c|c|c|c|}
\hline Programme Nano2017 & $\begin{array}{l}\text { STMicroelectronics } \\
\text { (STM) }\end{array}$ & $2014-2017$ & $\begin{array}{l}\text { STM et les entreprises } \\
\text { du secteur, laboratoires } \\
\text { publics. }\end{array}$ & $\begin{array}{l}\text { Mettre en production des filières } \\
\text { industrielles compétitives par rapport } \\
\text { aux USA, à Tä̈wan, à la Corée, être leader } \\
\text { sur la consommation énergétique en } \\
\text { numérique }(-30 \%)\end{array}$ \\
\hline $\begin{array}{l}\text { Consolidation d'un large écosystème } \\
\text { européen autour du site de Crolles }\end{array}$ & STM & Dès 2014 & $\begin{array}{l}\text { Industriels (STM et } \\
\text { entreprises du secteur), } \\
\text { laboratoires publics et } \\
\text { partenaires en Europe }\end{array}$ & $\begin{array}{l}\text { Elargir l'exploration de nouvelles } \\
\text { applications pour accroître la compétitivité } \\
\text { des acteurs européens en lançant } 4 \text { grands } \\
\text { programmes de coopération }\end{array}$ \\
\hline
\end{tabular}

Renforcer l'offre française de fonderies silicium et de services de conception

\begin{tabular}{|c|c|c|c|c|}
\hline $\begin{array}{l}\text { Fonderie silicium: renforcer l'offre } \\
\text { de ALTIS }\end{array}$ & Altis & mi 2014 & Altis & $\begin{array}{l}\text { Programme de R\&D au service de la } \\
\text { compétitivité et de l'attractivité }\end{array}$ \\
\hline $\begin{array}{l}\text { Création de plateformes de } \\
\text { conception/test/packaging couplées } \\
\text { aux centres de production (FabLabs) }\end{array}$ & & $3^{\mathrm{e}}$ trimestre 2014 & $\begin{array}{l}\text { Industriels de } \\
\text { l'ensemble } \\
\text { du secteur, laboratoires }\end{array}$ & $\begin{array}{l}\text { Valorisation des produits issus de la filière } \\
\text { nano auprès d'autres secteurs industriels }\end{array}$ \\
\hline
\end{tabular}

Assurer la disponibilité des composants revêtant un caractère stratégique pour les filières industrielles clés

\begin{tabular}{llll}
\hline $\begin{array}{l}\text { Organisation de la concertation } \\
\text { sectorielle sur la problématique ITAR }\end{array}$ & GIFAS & 2014-2015 & $\begin{array}{l}\text { Industriels des secteurs } \\
\text { concernés }\end{array}$ \\
\hline $\begin{array}{l}\text { Orientation accrue des achats de l'État } \\
\text { vers l'offre française et européenne }\end{array}$ & FGA-Etat & État & $\begin{array}{l}\text { Meilleur recours à l'offre nationale/ } \\
\text { européenne }\end{array}$ \\
\hline $\begin{array}{l}\text { Renforcement de l'offre industrielle } \\
\text { sur quelques familles de composants } \\
\text { critiques }\end{array}$ & $\begin{array}{l}\text { (lancement de } \\
\text { programme de } \\
\text { R\&D industrielle) }\end{array}$ & $\begin{array}{l}\text { EEV, Sofradir, Tronics, } \\
\text { Dolphin Integration, } \\
\text { SERMA et autres } \\
\text { entreprises privées, } \\
\text { BPIfr, CEA, CNRS, ONERA }\end{array}$ & $\begin{array}{l}\text { Capité d'exportation accrue dans } \\
\text { d'électrours fortement utilisateurs }\end{array}$ \\
& &
\end{tabular}

Faciliter l'accès aux innovations en composants

\begin{tabular}{|c|c|c|c|c|}
\hline $\begin{array}{l}\text { Partenariats verticaux dans la filière } \\
\text { pour la création de démonstrateurs }\end{array}$ & $\begin{array}{l}\text { Industriels } \\
\text { intégrateurs }\end{array}$ & $\begin{array}{l}\text { Début } 2015 \text { (appel à } \\
\text { projet) }\end{array}$ & $\begin{array}{l}\text { Industriels de } \\
\text { l'ensemble } \\
\text { du secteur }\end{array}$ & $\begin{array}{l}\text { Amélioration des relations entre } \\
\text { entreprises de la filière au service } \\
\text { de la R\&D }\end{array}$ \\
\hline $\begin{array}{l}\text { Mobilisation de fonds européens au } \\
\text { profit des PME }\end{array}$ & DGCIS et Jessica & Dès que possible & & $\begin{array}{l}\text { Amplifier l'action de Jessica au profit } \\
\text { des PME }\end{array}$ \\
\hline
\end{tabular}




\section{NANOÉLECTRONIQUE}
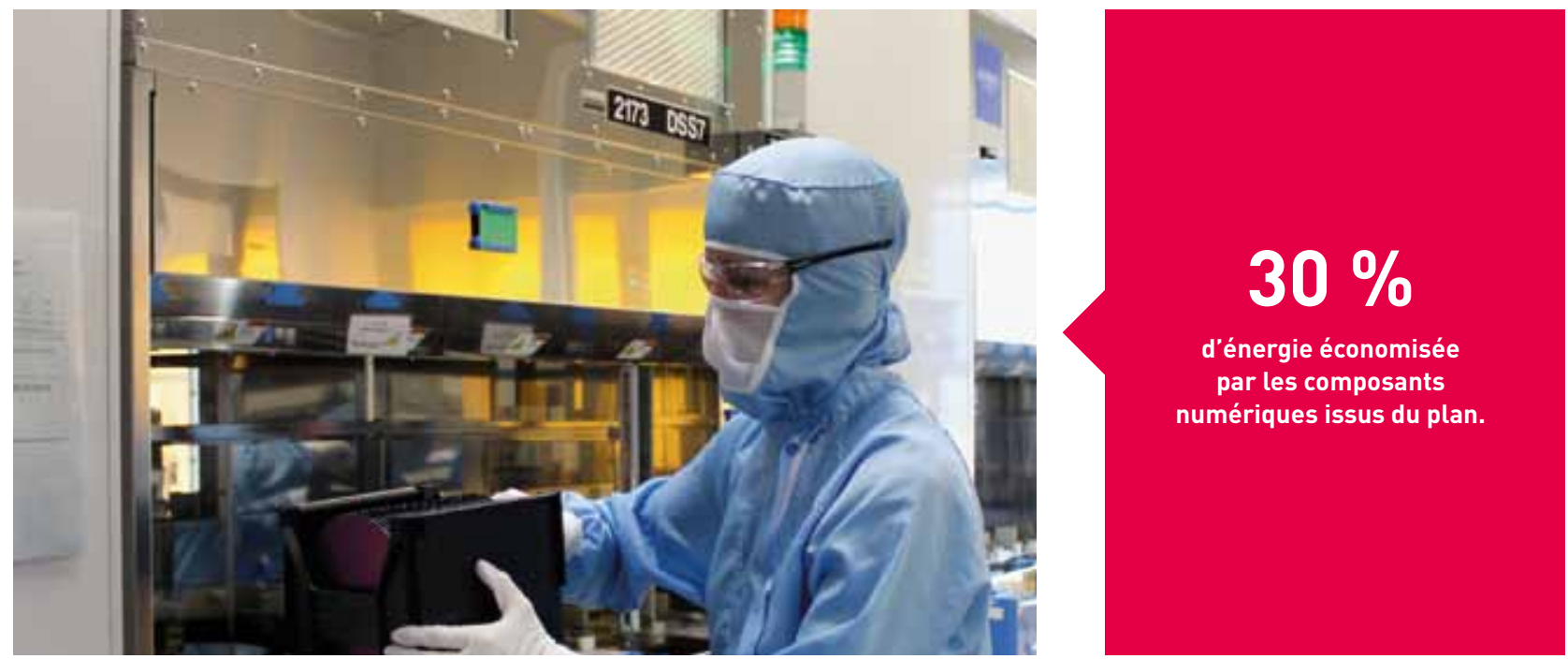

Usine de composants électroniques à Crolles (Isère)

Nous construisons la France de l'infiniment petit au service de tous. Les composants nanoélectroniques sont omniprésents dans notre quotidien : ils stockent l'information sur les clés USB, régissent nos ordinateurs, assurent la disponibilité des serveurs internet, déterminent la qualité des télévisions ou des consoles de jeux, permettent les services sans contact, contribuent à la sécurité de nos voitures comme au fonctionnement des pacemakers, optimisent la consommation énergétique des bâtiments ou nous aident à mesurer notre activité physique. Proposant de nouvelles fonctionnalités toujours plus intégrées, ils permettent de réinventer de nombreux secteurs pour lesquels ils sont stratégiques. - Les enjeux du plan sont triples, et associent de nombreux acteurs privés et publics. - Il s'agit d'une part d'assurer un leadership européen dans les technologies les plus avancées pour la production de composants intégrés numériques, qui irriguent la plupart des branches de l'industrie. Au travers du plan Nano2017, le site de Crolles (Isère) sera le plus avancé en Europe, offrant des gains importants en termes de performances et de consommation électrique ; son attractivité conduira à doubler sa capacité de production d'ici 2020. - Au-delà de ce socle technologique et industriel, le plan vise à assurer la disponibilité d'une offre de fonderie et de services associés, et à faciliter l'accès aux innovations en composants pour les industriels utilisateurs, tout en exploitant les compétences industrielles d'assemblage en électronique. Ces actions contribueront à fluidifier les relations entre acteurs de la filière et à accroître leur capacité à répondre aux besoins des marchés, tel que celui, émergent, des objets connectés. - Enfin, le plan cherche à assurer la disponibilité des composants stratégiques pour certaines filières industrielles clés, comme l'aéronautique, l'automobile ou la défense, dont ils conditionnent la capacité d'innovation et la compétitivité. 


\section{ÉQUIPE DU PLAN}

CHEF DE PROJET

Eric Carreel est PDG de Withings.

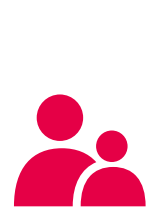

LES INDUSTRIELS

Eolane, Livosphere, CETIM, PEP, WE Network, FIEEC, Jessica/ Captronic, Cap Digital, Auchan, Carrefour, FNAC, Darty, Neodesign, Ouest Décolletage, Dedienne, SLTS, Mécareso, Process, Commeca, CDPlast, CNRFID, Urbanwave, Nexess, Editag, AFNIC

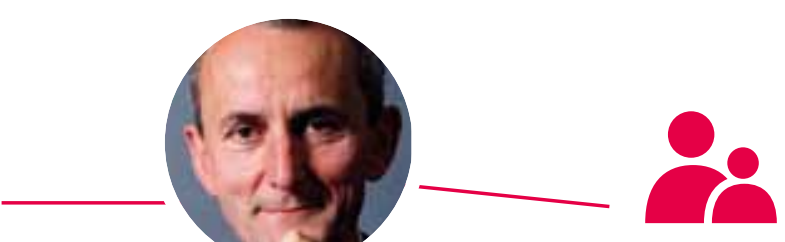

LES ACTEURS PUBLICS MEIN, DGCIS, DIRECCTE Pays de la Loire, CGI, Mission French Tech, MENESR, CGSP, Bpifrance, Communauté d'agglomération d'Angers Loire Métropole et Conseil régional des Pays de la Loire

\section{SYNTHĖSE DES ACTIONS DU PLAN}

\begin{tabular}{|c|c|c|c|c|}
\hline Action & Pilote & Calendrier & Acteurs & Finalités/livrables \\
\hline $\begin{array}{l}\text { Mettre en place } \\
\text { une première } \\
\text { cité de l'objet } \\
\text { connecté }\end{array}$ & Eolane & $\begin{array}{l}\text { Septembre } 2014: \text { Cahier } \\
\text { des charges } \\
\text { Année } 1: \text { : Espaces } \\
\text { Co-working, Fablab et } \\
\text { Innovation industrielle } \\
\text { Année } 3: \text { Production } \\
\text { industrielle }\end{array}$ & $\begin{array}{l}\text { Industriels: Ouest Decolletage, } \\
\text { Dedienne, SLTS, Mécareso, Process, } \\
\text { Commeca, CD Plast, Hubert Chevalier } \\
\text { Acteurs publics: collectivités } \\
\text { territoriales; DIRECCTE Pays de la } \\
\text { Loire, CGI, Mission French Tech }\end{array}$ & $\begin{array}{l}\text { Création d'un site industriel propice au } \\
\text { développement et à la production, en France, } \\
\text { d'objets connectés innovants }\end{array}$ \\
\hline $\begin{array}{l}\text { Stimuler } \\
\text { l'émergence } \\
\text { d'une offre } \\
\text { française }\end{array}$ & $\begin{array}{l}\text { Bpifrance, } \\
\text { CGI, MEIN } \\
\text { Une } \\
\text { entreprise } \\
\text { pilotes à } \\
\text { identifier }\end{array}$ & $\begin{array}{l}2015: \text { Mise en place des } \\
\text { prêts à l'industrialisation }\end{array}$ & $\begin{array}{l}\text { Banques, Bpifrance, fonds de } \\
\text { garanties régionaux, CGI, MEIN } \\
\text { Assurances, Ministère des affaires } \\
\text { sociales et de la santé: étude des } \\
\text { liens entre l'utilisation d'outils } \\
\text { innovants et la santé }\end{array}$ & $\begin{array}{l}\text { 1/Mise en place de prêts à l'industrialisation et } \\
\text { d'outils facilitants l'accès aux financements pour les } \\
\text { entreprises qui produisent en France } \\
\text { 2/Développement de la commande publique } \\
\text { et privée innovante pour les objets connectés. } \\
\text { Développement du Corporate Wellness }\end{array}$ \\
\hline $\begin{array}{l}\text { Promouvoir } \\
\text { l'offre } \\
\text { française }\end{array}$ & $\begin{array}{l}\text { Enseignes } \\
\text { de grande } \\
\text { distribution } \\
\text { Mission } \\
\text { French Tech }\end{array}$ & $\begin{array}{l}\text { Calendrier propre à chaque } \\
\text { enseigne. } \\
\text { Mi-2015 : Première } \\
\text { « Keynote» intégrant des } \\
\text { annonces sur le lancement } \\
\text { d'objets connectés French } \\
\text { Tech }\end{array}$ & $\begin{array}{l}\text { Auchan, Carrefour, FNAC, Darty, Lick, } \\
\text { Mission French Tech } \\
\text { Entreprises de la French Tech : } \\
\text { Netatmo, Withings, Parrot, } \\
\text { Medissimo, Sen.se, Sigfox, Holî... } \\
\text { French Tech, CGI, MEIN, AFII }\end{array}$ & $\begin{array}{l}\text { 1/Mettre en place des show rooms sur les objets } \\
\text { connectés pour mettre en avant la French Tech } \\
\text { auprès des consommateurs et des voyageurs } \\
\text { 2/Organiser un évènement de visibilité international } \\
\text { mettant en avant l'excellence française }\end{array}$ \\
\hline $\begin{array}{l}\text { Déployer } \\
\text { un réseau } \\
\text { européen pour } \\
\text { l'internet des } \\
\text { objets }\end{array}$ & Sigfox & $\begin{array}{l}\text { Déploiement progressif d'un } \\
\text { réseau pan-Européen }\end{array}$ & Industriels français et européens & $\begin{array}{l}\text { Réseau pan-Européen ( } 28 \text { pays) dédié à l'internet } \\
\text { des objets } \\
\text { Standardisation } 5 G\end{array}$ \\
\hline \multirow{2}{*}{$\begin{array}{l}\text { Mettre en } \\
\text { œuvre le } \\
\text { déploiement } \\
\text { d'objets } \\
\text { connectés } \\
\text { à l'échelle } \\
\text { de filières } \\
\text { industrielles }\end{array}$} & \multirow{2}{*}{$\begin{array}{l}\text { CNRFID } \\
1 \text { chef de } \\
\text { projet par } \\
\text { filière }\end{array}$} & 9 mois de travaux & $\begin{array}{l}\text { CNRFID, GS1, Orange, EDF, Viaméca. } \\
\text { autres acteurs privés à mobiliser. } \\
\text { Co-financement public des travaux } \\
\text { Participation des pôles de } \\
\text { compétitivité }\end{array}$ & $\begin{array}{l}1 / \text { Projets pilotes dans cinq filières clés pour } \\
\text { construire des projets ambitieux de renforcement } \\
\text { de la traçabilité : alimentaire, luxe, énergie, } \\
\text { mécanique, une filière à identifier }\end{array}$ \\
\hline & & $\begin{array}{l}\text { Finalisation et lancement } \\
\text { sur la base des résultats } \\
\text { des projets pilotes dans les } \\
5 \text { filières pionnières }\end{array}$ & $\begin{array}{l}\text { PME et grands groupes de chaque } \\
\text { filière avec au besoin un co- } \\
\text { financement public }\end{array}$ & $\begin{array}{l}\text { 2/Définition d'au moins } 3 \text { projets majeurs } \\
\text { d'utilisation des objets connectés à l'échelle d'une } \\
\text { filière industrielle (renforcement de la traçabilité, } \\
\text { gestion des flux logistiques, marquage d'outils...) }\end{array}$ \\
\hline $\begin{array}{l}\text { Créer un label } \\
\text { pour les objets } \\
\text { et procédés } \\
\text { dont l'impact } \\
\text { sur la vie } \\
\text { privée des } \\
\text { utilisateurs est } \\
\text { maitrisé }\end{array}$ & $\begin{array}{l}\text { Association } \\
\text { adossée au } \\
\text { CNRFID }\end{array}$ & $\begin{array}{l}\text { Référentiel de labellisation : } \\
\text { juin } 2014 \text { - mars } 2015 \\
\text { Centre de labellisation : } \\
\text { janvier } 2016\end{array}$ & $\begin{array}{l}\text { Fournisseurs de technologies, } \\
\text { Fédérations professionnelles, } \\
\text { Opérateurs/Utilisateurs d'application } \\
\text { RFID... } \\
\text { Financement public sollicité pour la } \\
\text { mise en place du référentiel du label }\end{array}$ & $\begin{array}{l}\text { Label identifiant les objets et procédés dont l'impact } \\
\text { sur la vie privée des utilisateurs est maitrisé }\end{array}$ \\
\hline
\end{tabular}




\section{OBJETS CONNECTÉS}

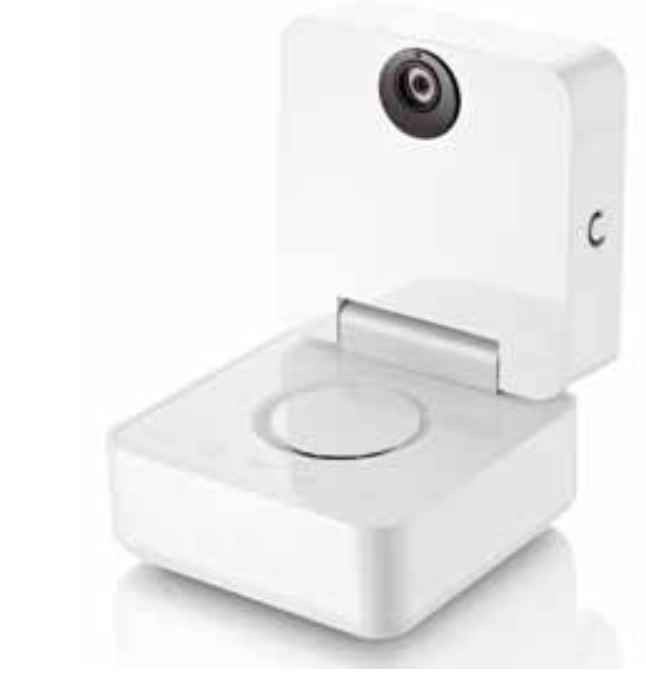

Smart baby monitor, Withings
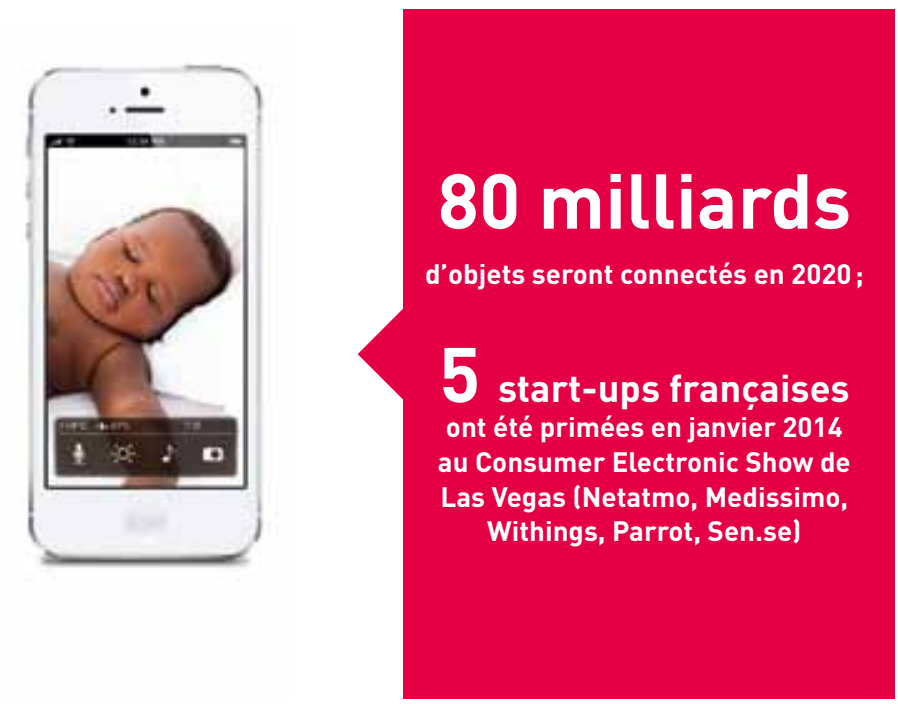

- Nous construisons la France des objets communicants. - Avec des entreprises telles que Medissimo, Myfox, Netatmo, Parrot, Sen.se, Sigfox ou Withings, l'expertise française dans le domaine des objets connectés est aujourd'hui reconnue. À l'étranger, la multiplication des objets connectés s'accompagne d'une massification des investissements. Dans cette période charnière, la France doit rester parmi les leaders. Elle doit pour cela renforcer son savoir-faire... et le faire savoir! - Le rapport intime qui se crée entre objets et services pousse les entreprises à s'adapter aux nouveaux usages et les engage dans une course effrénée à l'innovation. Le raccourcissement des cycles d'innovation constitue une opportunité pour la localisation d'usines en France, au plus près des équipes de développement. - Sous l'impulsion d'Eolane et d'industriels locaux, une première cité de l'objet connecté sera mise en place dès 2014 à Angers. Pour concevoir et produire les objets les plus innovants, cette cité rassemblera un fablab, un centre d'innovation technologique et des usines de production. $15 \mathrm{M} €$ d'investissement et 50 personnes seront nécessaires au fonctionnement de cette cité d'un nouveau type, propice au croisement des expertises électroniques et mécaniques. - Les objets connectés French Tech doivent être demain connus et reconnus, du consommateur français au journaliste américain. 4 enseignes de distribution se mobilisent pour mettre en avant ces objets. 2 d'entre elles s'engagent en outre à faciliter la commercialisation des objets les plus innovants. - Des problèmes techniques devront être surmontés pour permettre le raccordement de 80 milliards d'objets en 2020. Le projet de réseau européen porté par Sigfox constituera une des réponses. - Appliqués aux filières industrielles, les objets connectés offrent des applications nombreuses : renforcement de la traçabilité, marquage d'outils... Pour faire émerger des champions français, il est proposé de construire des projets, non pas à l'échelle d'entreprises, mais à l'échelle de filières. L'alimentation, le luxe, l'énergie et les industries mécaniques sont d'ores et déjà identifiées. 


\section{ÉQUIPE DU PLAN}

CHEF DE PROJET

Vincent Marcatté est vice-président Open Innovation d’Orange Labs, président du pôle Images \& Réseaux et président de l'IRT B-Com

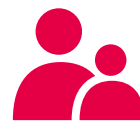

LES INDUSTRIELS

Laster Technologies, Optinvent, Orange, Dassault Systèmes, Immersion/AFRV, Artefacto, Total Immersion

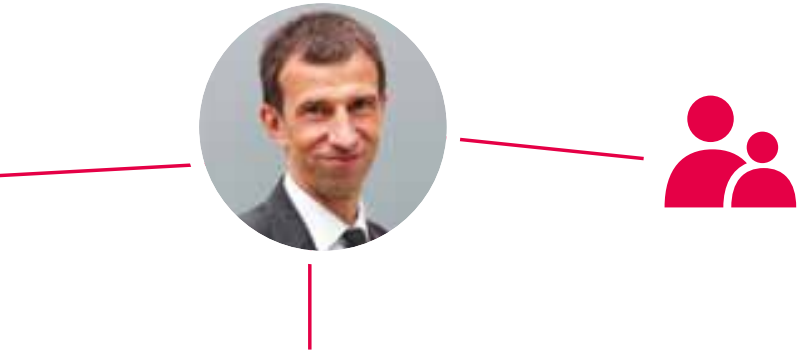

LES ACTEURS PUBLICS MEIN, MEDDE, ADEME, CRE, DGCIS, BPIfrance

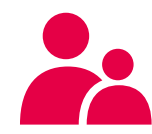

LES ÉCOLES ET ORGANISMES DE RECHERCHE

IRT B-com

\section{SYNTHÈSE DES ACTIONS DU PLAN}

\begin{tabular}{|c|c|c|c|c|c|}
\hline Action & Pilote & Calendrier & $\begin{array}{l}\text { Acteurs } \\
\text { privés }\end{array}$ & $\begin{array}{l}\text { Acteurs publics } \\
\text { et leviers d'action }\end{array}$ & Finalités/livrables \\
\hline \multicolumn{6}{|c|}{ Accélérer le développement de projets industriels matures grâce à des initiatives centrées sur les usages } \\
\hline $\begin{array}{l}\text { Première vague de projets } \\
\text { présélectionnés }\end{array}$ & $\begin{array}{l}\text { Orange } \\
\text { B-Com } \\
\text { Diotasoft } \\
\text { Artefacto }\end{array}$ & $\begin{array}{l}\text { Premiers } \\
\text { projets lancés } \\
\text { en } 2014 \text { avec } \\
\text { résultats en } \\
2015\end{array}$ & $\begin{array}{l}\text { Dassault } \\
\text { Aviation } \\
\text { Theoris } \\
\text { Total } \\
\text { Immersion } \\
\text { Voxygen }\end{array}$ & $\begin{array}{l}\text { MEIN, BPIfrance, CGI } \\
\text { CHU de Rennes } \\
\text { et CHU de Brest } \\
\text { Rennes Metropole }\end{array}$ & $\begin{array}{l}\text { Plusieurs projets pilotes emblématiques à résultats } \\
\text { visibles rapidement seront lancés afin de démontrer } \\
\text { les possibilités offertes par la réalité augmentée (visite } \\
\text { augmentée du musée d'Orsay, voix augmentée dans la } \\
\text { santé, Réalité augmentée projective dans l'aéronautique, } \\
\text { ville augmentée à Rennes) }\end{array}$ \\
\hline $\begin{array}{l}\text { Appel à manifestation } \\
\text { d'intérêt permettant de } \\
\text { sélectionner la seconde vague }\end{array}$ & MEIN & 2015 & $\begin{array}{l}\text { " French } \\
\text { Augmented } \\
\text { Reality " }\end{array}$ & $\begin{array}{l}\text { BPIfrance, CGI, pôles } \\
\text { de compétitivité }\end{array}$ & $\begin{array}{l}\text { Aide à la mutation de l'appareil productif français et } \\
\text { encouragement d'applications à fortes valeurs applicative } \\
\text { et démonstrative }\end{array}$ \\
\hline \multicolumn{6}{|c|}{ Mettre en place une plateforme ouverte Réalité augmentée Factory } \\
\hline $\begin{array}{l}\text { Plateforme ouverte Réalité } \\
\text { augmentée Factory, servant } \\
\text { de référence pour la } \\
\text { standardisation }\end{array}$ & $\begin{array}{l}\text { MEIN } \\
\text { Dassault } \\
\text { Systèmes, } \\
\text { B-com, } \\
\text { Rmn-GP }\end{array}$ & $\begin{array}{l}1 \text { er semestre } \\
2015: \\
\text { lancement } \\
\text { Réalité } \\
\text { augmentée } \\
\text { Factory }\end{array}$ & $\begin{array}{l}\text { Orange, } \\
\text { Artefacto, } \\
\text { Robocortex, } \\
\text { Logilab, } \\
\text { Zengularity }\end{array}$ & $\begin{array}{l}\text { Rennes Métropole, } \\
\text { IGN } \\
\text { MEIN, BPIfrance, CGI, } \\
\text { CMN } \\
\text { Télécom Sud Paris, } \\
\text { Institut Mines Telecom }\end{array}$ & $\begin{array}{l}\text { Mise à disposition de briques technologiques, de services } \\
\text { d'expérimentations, d'infrastructures et de bases de } \\
\text { données afin de faciliter la réalisation d'applications } \\
\text { utilisant la Réalité augmentée. Secteurs visés au départ : } \\
\text { urbanisme, communication interpersonnelle, et contexte } \\
\text { muséal }\end{array}$ \\
\hline \multicolumn{6}{|c|}{ Réalité augmentée industrielle dans le domaine de l'aéronautique } \\
\hline $\begin{array}{l}\text { Fourniture d'une assistance } \\
\text { Réalité augmentée aux usines } \\
\text { aéronautiques }\end{array}$ & $\begin{array}{l}\text { IRT Jules } \\
\text { Verne, IRT } \\
\text { B-com, } \\
\text { Dassault } \\
\text { Systèmes }\end{array}$ & & $\begin{array}{l}\text { Airbus, } \\
\text { Aérolia, Stx, } \\
\text { Clarté }\end{array}$ & MEIN, CGI, BPIfrance & $\begin{array}{l}\text { Les tâches productives visées sont relatives } \\
\text { à l'assemblage, au montage, au contrôle qualité, à la } \\
\text { maintenance industrielle. } \\
\text { Deux applications seront plus particulièrement ciblées : } \\
\text { le contrôle qualité avec relocalisation sans marqueur } \\
\text { et un guide d'instruction de montage de structures } \\
\text { complexes }\end{array}$ \\
\hline \multicolumn{6}{|c|}{ Créer les écosystèmes et la gouvernance associée, valoriser le savoir-faire } \\
\hline $\begin{array}{l}\text { Création du groupe «FRENCH } \\
\text { AUGMENTED REALITY» (FAR) } \\
\text { et création d'un observatoire } \\
\text { du marché }\end{array}$ & $\begin{array}{l}\text { Images et } \\
\text { Réseaux }\end{array}$ & 2014 & $\begin{array}{l}\text { IRT B-Com et } \\
\text { Jules Verne, } \\
\text { AFRV, } \\
\text { AR Pro, Laval } \\
\text { Virtual, } \\
\text { NUMA, } \\
\text { Orange, } \\
\text { Immersion }\end{array}$ & $\begin{array}{l}\text { FrenchTech, pôles de } \\
\text { compétitivité } \\
\text { (Cap Digital, } \\
\text { Imaginove, } \\
\text { Systematic, } \\
\text { PICOM) } \\
\text { Etalab, BPIfrance }\end{array}$ & $\begin{array}{l}\text { - Mise en relation et coopération entre acteurs } \\
\text { académiques et économiques } \\
\text { - Favorisation de l'émergence de champions } \\
\text { - Concertation sur la standardisation et la réglementation } \\
\text { - Mise en place d'un annuaire des acteurs du secteur } \\
\text { - Facilitation du recrutement et de la formation } \\
\text { - Animation de la communauté des développeurs et } \\
\text { créateurs de start-ups }\end{array}$ \\
\hline
\end{tabular}




\section{RÉALITÉ AUGMENTÉE}
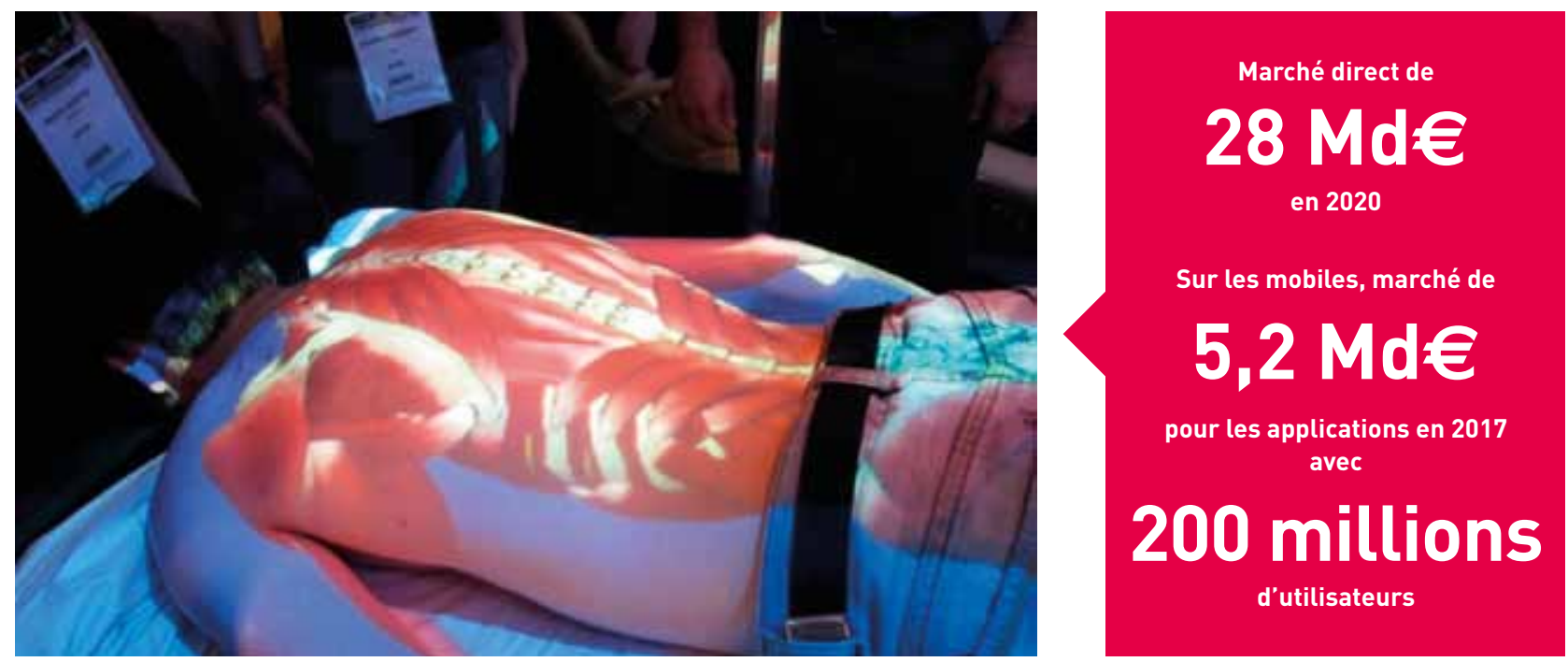

Exemple de réalité augmentée à usage médical

- Nous construisons la France où le virtuel s'ajoute au réel, pour plus de services personnalisés. En superposant des informations et images virtuelles à notre perception naturelle, la réalité augmentée révolutionne notre vie quotidienne et ouvre pour tous et dans tous les domaines, des perspectives nouvelles en termes d'amélioration des services et des procédés. - Sur ce marché, la France s'appuie sur de nombreux grands groupes et PME innovantes, producteurs de contenus, fabricants de matériels, concepteurs de logiciels. Afin de bénéficier pleinement de la croissance de ce marché et pour en démultiplier les opportunités, le plan Réalité augmentée vise à accélérer la diffusion de la technologie «Made in France » dans les usages professionnels et grand public. Conjuguant l'excellence française dans l'aéronautique, l'usine du futur, la culture, la santé et l'urbanisme, à notre savoir-faire sur la réalité augmentée, des projets industriels à forte valeur applicative seront lancés dès 2014 sur ces thématiques, associant grands groupes nationaux et pépites technologiques. - Afin d'encourager les partenariats futurs et d'aider à la mutation de l'appareil productif français, notamment dans les secteurs du commerce et du jeu vidéo, un appel à manifestation d'intérêt sera lancé en 2015. Pour répondre aux enjeux de standardisation, faciliter la diffusion de nos technologies et le développement de services s'appuyant sur la réalité augmentée, la mutualisation des moyens et l'interopérabilité seront encouragées. - Nous mettrons pour cela en place une plateforme collaborative mettant à disposition de l'écosystème des créateurs d'applications : des bases de données urbaines et iconographiques, des briques technologiques et des infrastructures. Des actions de communication seront déployées pour sensibiliser à l'apport de la réalité augmentée, susciter des partenariats et encourager la diffusion du numérique dans les entreprises. Les leviers réglementaires nécessaires à l'essor du marché de la réalité augmentée seront actionnés. 


\section{ÉQUIPE DU PLAN}

CHEF DE PROJET

Olivier Piou est directeur général de Gemalto.

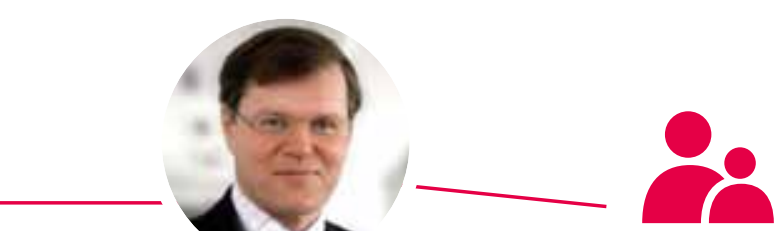

LES ACTEURS PUBLICS Mission FrenchTech, MEIN, MEDDE (AFIMB), Communautés Urbaines de Strasbourg et Bordeaux, Bpifrance, UBI France, $\mathrm{CGI}, \mathrm{CCl}$

\section{SYNTHÈSE DES ACTIONS DU PLAN}

\begin{tabular}{|c|c|c|c|}
\hline Action & Pilote et acteurs impliqués & Calendrier & Objectifs en 2020 \\
\hline $\begin{array}{l}\text { Généralisation du paiement } \\
\text { sans contact: } \\
\text { - diffusion massive des cartes } \\
\text { - déploiement dans les TPE } \\
\text { - communication vers les commerçants } \\
\text { et consommateurs }\end{array}$ & $\begin{array}{l}\text { GIE CB, Mercatel, banques, } \\
\text { enseignes de grande distribution, } \\
\text { opérateurs mobile, DGFIP, DG Trésor, } \\
\text { DGCIS, CGI, BPI France }\end{array}$ & D’ici 2020 & $\begin{array}{l}100 \% \text { des cartes et des terminaux } \\
\text { de paiement sont sans contact. } \\
55 \% \text { de paiements en espèces en valeur, } \\
\text { sont remplacés par des paiements } \\
\text { par carte } \\
8 \text { millions de personnes paient } \\
\text { avec leurs mobiles }\end{array}$ \\
\hline $\begin{array}{l}\text { Massification de la billettique transport } \\
\text { sur support mobile : } \\
\text { - diffusion d'une application nationale } \\
\text { interopérable } \\
\text { - élaboration d'une solution d'émission } \\
\text { de titres }\end{array}$ & $\begin{array}{l}\text { SNCF, opérateurs } \\
\text { de transport, opérateur mobile, } \\
\text { autorités organisatrices des } \\
\text { transports, AFIMB, DGCIS }\end{array}$ & D’ici 2020 & $\begin{array}{l}\text { Une application de billettique interopérable } \\
\text { est implémentée dans } 50 \% \text { des villes } \\
\text { de plus de } 200000 \text { habitants } \\
20 \% \text { des titres de transport sont achetés } \\
\text { sur mobile }\end{array}$ \\
\hline $\begin{array}{l}\text { Développement et déploiement } \\
\text { des services sans contact mobiles } \\
\text { pour le commerce } \\
\text { ( } 2 \text { expérimentations à grande échelle) }\end{array}$ & $\begin{array}{l}\text { Fournisseurs de solutions } \\
\text { dans la grande distribution, } \\
\text { enseignes (grande distribution } \\
\text { et restauration rapide), } \\
\text { DGCIS, CGI, BPI France }\end{array}$ & Pilotes dès 2015 & $\begin{array}{l}\text { Au sein des enseignes équipées, } \\
\text { au moins } 20 \% \text { des clients utilisent } \\
\text { la solution mobile sans contact }\end{array}$ \\
\hline $\begin{array}{l}\text { Facilitation du déploiement } \\
\text { par les territoires des services } \\
\text { sans contact pour les villes } \\
\text { et les citoyens: } \\
\text { - étude de faisabilité } \\
\text { - développement et déploiement } \\
\text { de la solution mutualisée }\end{array}$ & $\begin{array}{l}\text { SGMAP, Collectivités territoriales, } \\
\text { MDREFP, DGCIS, CGI, BPI France, } \\
\text { industriels fournisseurs de solutions }\end{array}$ & D’ici 2020 & $\begin{array}{l}\text { Solution de déploiement des services } \\
\text { aux citoyens développée et adoptée par au } \\
\text { moins } 10 \text { collectivité }\end{array}$ \\
\hline $\begin{array}{l}\text { Promotion de l'interopérabilité } \\
\text { à travers une plateforme de test } \\
\text { de bout-en-bout }\end{array}$ & $\begin{array}{l}\text { Pôle SCS, GIE-CB, } \\
\text { Laboratoires de test, } \\
\text { fournisseurs de solutions } \\
\text { et matériels sans contact, } \\
\text { DGCIS, CGI, BPI France }\end{array}$ & $\begin{array}{l}\text { D'ici fin } 2015 \\
\text { (déploiement } \\
\text { de la plateforme) }\end{array}$ & Exportation des services de la plateforme \\
\hline $\begin{array}{l}\text { Dynamisation de l'écosystème } \\
\text { et soutien aux startups: } \\
\text { - concours de startups chaque année } \\
\text { - programme d'accompagnement }\end{array}$ & $\begin{array}{l}\text { Orange, industriels impliqué } \\
\text { dans les services sans contact } \\
\text { (banque, grande distribution, } \\
\text { transport) DGCIS, CGI, BPI France }\end{array}$ & A partir de 2014 & $\begin{array}{l}50 \text { startups dans le programme } \\
20 \text { ont été accompagnées avec succès } \\
\text { à l'exportation }\end{array}$ \\
\hline
\end{tabular}




\section{SERVICES SANS CONTACT}
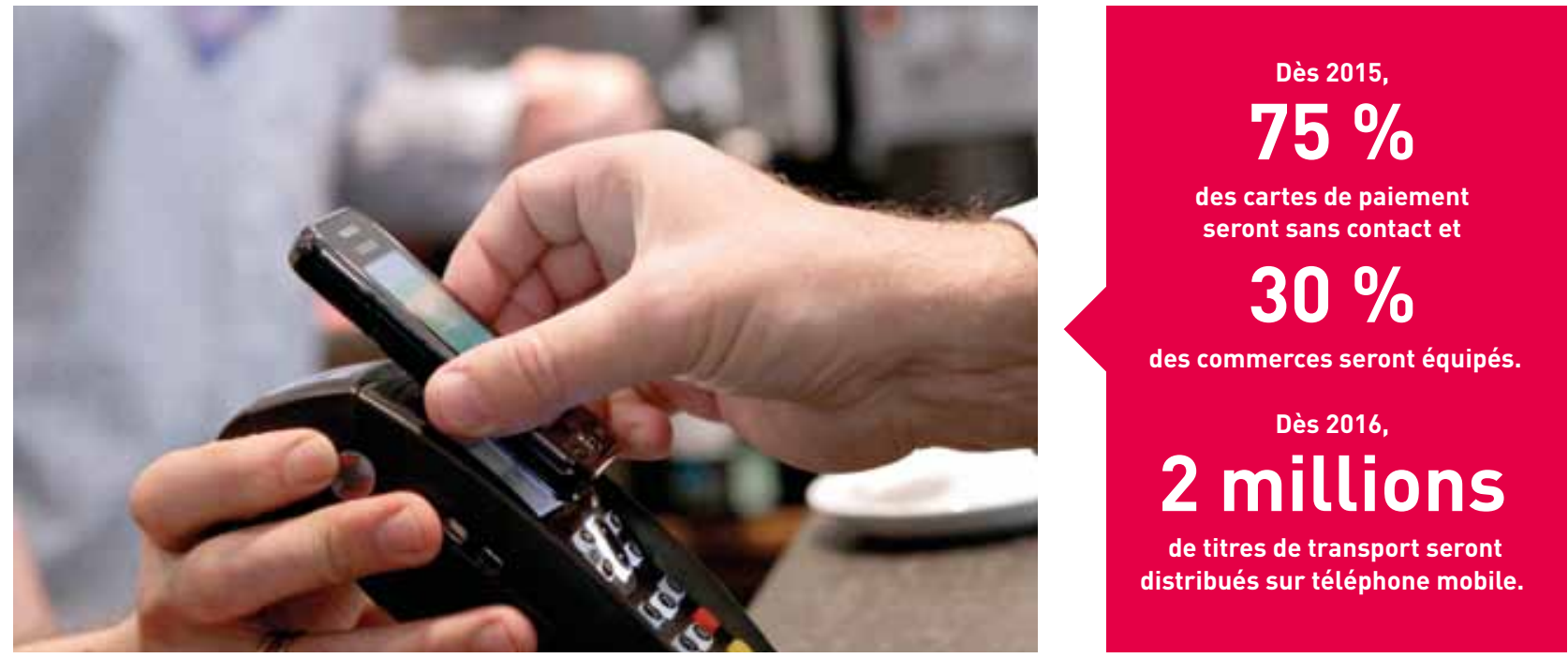

Paiement avec smartphone

- Nous construisons la France des villes intelligentes où la vie devient plus simple. Les services sans contact s'intègrent dans le « bouquet » d'applications et services numériques de la ville intelligente: gestion de la fluidité de la mobilité et des échanges, confort et sécurité de l'usager de la ville. La France, aujourd'hui le leader mondial des technologies sans contact sécurisées, cartes et mobiles, doit relever le défi de la concurrence globale. - Le plan Services sans contacts prévoit de développer massivement l'interopérabilité de la billettique dans les transports. Les voyageurs pourront acheter et valider un seul ticket de transport dématérialisé du début à la fin de leur parcours, sur leur mobile. Les usagers des services de la ville pourront également gérer leurs demandes, échanges et interactions via une plateforme mutualisée de services aux citoyens, plus économe en ressources. Dans les commerces physiques, d'un simple «TAP» par carte ou mobile sur un terminal de paiement ou une étiquette NFC, le client évite l'attente aux caisses, reçoit des informations sur les produits, des offres personnalisées. - Pour que ces bénéfices s'inscrivent dans une équation économique viable, le plan Services sans contacts a défini cinq objectifs à atteindre dans les 24 mois : mobiliser l'ensemble des acteurs vers l'objectif commun du déploiement massif, faciliter le déploiement des services, notamment sur mobile via des plateformes NFC mutualisées (test, transport, commerce, services de la ville), déployer systématiquement les standards nationaux de référence paiement et transport, communiquer dans les territoires pour favoriser l'adoption de ces nouveaux usages, et enfin, créer un écosystème industriel en accompagnant les startups et les PME. 


\section{ÉQUIPE DU PLAN}

CHEF DE PROJET

Gérard Roucairol est président de Teratec.

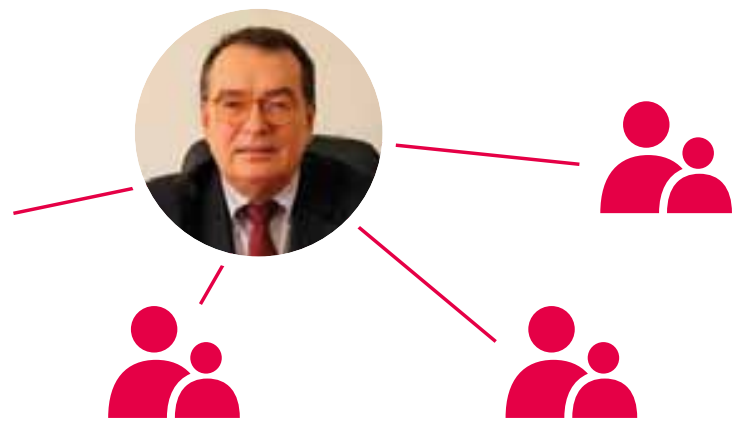

LES ACTEURS PUBLICS Agglomération d'Orléans, Bpifrance, CGI, DGA, DGCIS, MENESR, GENCI, Région Centre

Air liquide, Airbus, Bull, Caps Entreprise, Cybeletech, Dassault Aviation, Distene, ESI-Group, L'Oréal, Limagrain, Michelin, Numtech, Plateforme de la

Filière Automobile, Safran, Scilab Enterprises, Sofiproteol, Ubisoft
LES PÔLES DE COMPÉTITIVITÉ Advancity, Aerospace Valley, Alsace BioValley, Cap Digital, Lyonbiopôle, Medicen, Systematic
LES ÉCOLES ET ORGANISMES DE RECHERCHE

CEA, École Polytechnique, INPG, INRIA

\section{SYNTHĖSE DES ACTIONS DU PLAN}

\begin{tabular}{|c|c|c|c|}
\hline Action & Pilote & Calendrier & Finalités/livrables \\
\hline \multicolumn{4}{|l|}{ Maîtrise des technologies critiques } \\
\hline $\begin{array}{l}\text { Développement de la nouvelle génération } \\
\text { de supercalculateurs exaflopiques }\end{array}$ & CEA & Avril 2014 - décembre 2015 & $\begin{array}{l}\text { Capacité à concevoir en France des } \\
\text { supercalculateurs en l'état de l'art mondial }\end{array}$ \\
\hline $\begin{array}{l}\text { Création de centres de recherche } \\
\text { industrielle sur les nouvelles architectures } \\
\text { matériel-logiciel }\end{array}$ & Teratec & Second semestre 2014 & Mise en place de centres de recherche industriels \\
\hline $\begin{array}{l}\text { Projet de recherche sur les nouvelles } \\
\text { architectures matériel-logiciel }\end{array}$ & Bull & Courant 2015 & $\begin{array}{l}\text { Projet structurant de R\&D sur les architectures } \\
\text { matériel-logiciel }\end{array}$ \\
\hline $\begin{array}{l}\text { Développement de la nouvelle génération } \\
\text { de logiciels }\end{array}$ & $\begin{array}{l}\text { Éditeurs de logiciels } \\
\text { (ESI-Group Scilab } \\
\text { Enterprises, etc.) }\end{array}$ & Courant 2014 & Projet structurant de R\&D sur le logiciel HPC \\
\hline \multicolumn{4}{|l|}{ Initiatives sectorielles } \\
\hline Simulation pour la santé & Teratec & $1^{\text {er }}$ trimestre 2015 & $\begin{array}{l}\text { Projets structurants «Santé et Calcul haute } \\
\text { performance» }\end{array}$ \\
\hline $\begin{array}{l}\text { Modélisation et simulation des Systèmes } \\
\text { urbains }\end{array}$ & $\begin{array}{l}\text { Teratec - Advancity - } \\
\text { Systematic } \\
\text { Bull }\end{array}$ & $\begin{array}{l}\text { Courant } 2014 \\
\text { Courant } 2015\end{array}$ & $\begin{array}{l}\text { Grands projets structurants «Logiciels Systèmes } \\
\text { Urbains» }\end{array}$ \\
\hline Technologies numériques pour le végétal & $\begin{array}{l}\text { Limagrain, Sofiproteol, } \\
\text { Agglomération } \\
\text { d’Orléans, Région } \\
\text { Centre }\end{array}$ & Courant 2014 & $\begin{array}{l}\text { - Grand projet structurant «PLM du Végétal » } \\
\text { - Mise en place de la structure d'animation } \\
\text { et de promotion Vegetal Valley }\end{array}$ \\
\hline Outils logiciels pour l'étude des matériaux & $\begin{array}{l}\text { Michelin, Safran, } \\
\text { Airbus, Air Liquide, } \\
\text { CEA, Plateforme de la } \\
\text { Filière Automobile }\end{array}$ & Courant 2014 & Mise en place d'un laboratoire industriel \\
\hline $\begin{array}{l}\text { Technologies numériques pour les industries } \\
\text { manufacturières et l'énergie }\end{array}$ & Dassault-Aviation, & Courant 2014 & $\begin{array}{l}\text { Mise en place d'un laboratoire industriel } \\
\text { dans le cadre de l'IRT System X. }\end{array}$ \\
\hline Technologies HPC pour le multimédia & $\begin{array}{l}\text { Ubisoft, Teratec, Cap } \\
\text { Digital }\end{array}$ & Courant 2014 & Grand projet structurant «Image et Cinéma » \\
\hline \multicolumn{4}{|l|}{ Diffusion de la simulation vers les PME et ETI } \\
\hline Diffusion dans le tissu industriel & CGI, DGCIS & Courant 2014 & $\begin{array}{l}\text { Mise en place d'une structure d'accompagnement } \\
\text { des PME/ETI }\end{array}$ \\
\hline \multicolumn{4}{|l|}{ Formation } \\
\hline $\begin{array}{l}\text { Formation de spécialistes et des futurs } \\
\text { utilisateurs }\end{array}$ & $\begin{array}{l}\text { ENSIMAG, École } \\
\text { Polytechnique }\end{array}$ & $\begin{array}{l}\text { Selon les procédures et } \\
\text { les calendriers du MENESR } \\
\text { et des plans des universités } \\
\text { et grandes écoles MENESR }\end{array}$ & $\begin{array}{l}\text { Initiatives spécifiques MENESR pour la structure } \\
\text { nationale de coordination }\end{array}$ \\
\hline
\end{tabular}




\section{SUPERCALCULATEURS}
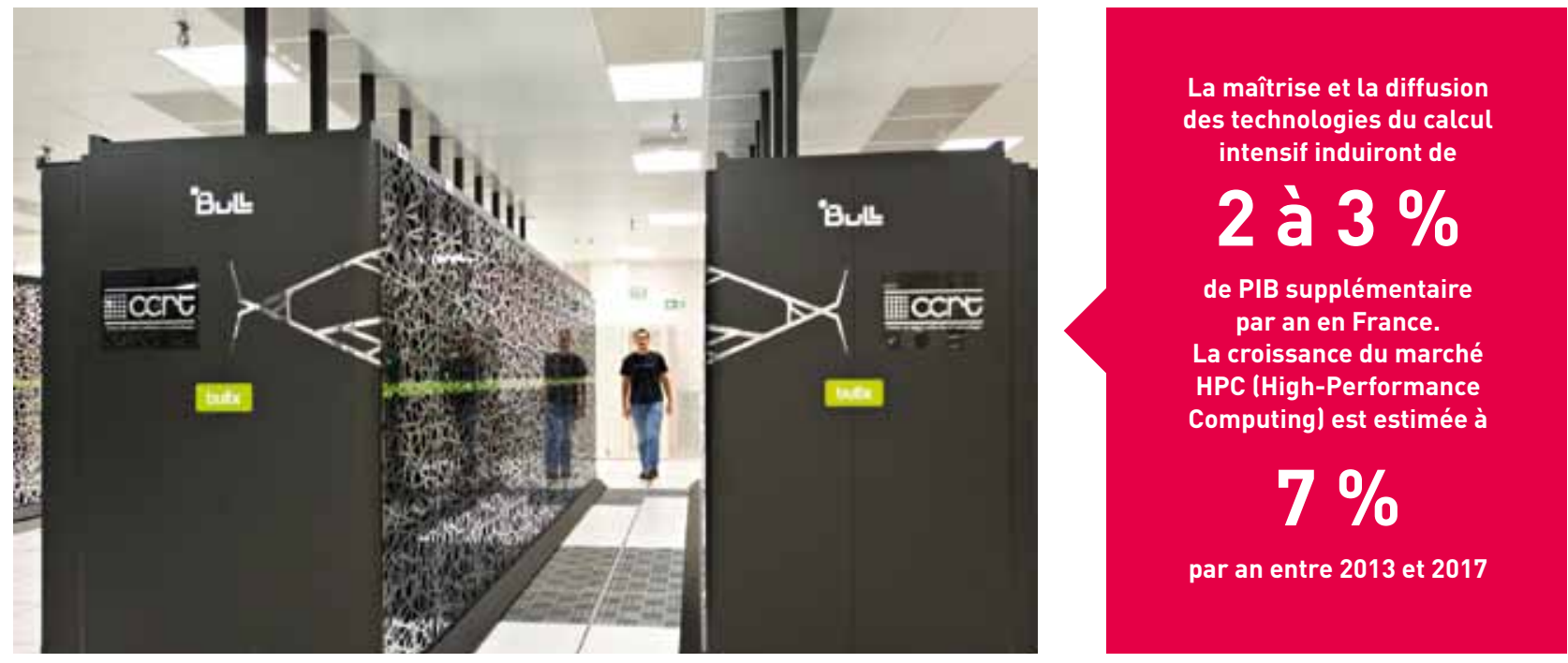

Supercalculateurs, Bull

- Nous construisons la France de la puissance de calcul et de la simulation numérique. Le calcul intensif ou « haute performance» fait partie des technologies génériques critiques jouant un rôle moteur pour l'innovation dans l'ensemble des secteurs de l'industrie et des services (industries manufacturières, énergie, santé, environnement, végétal, multimédia, etc.). Le recours croissant à la modélisation et à la simulation numérique serait inconcevable sans les performances offertes par ces technologies matérielles et logicielles. L'augmentation exponentielle des puissances de calcul et la disponibilité des nouvelles générations de logiciels applicatifs permettent une multiplication de nouveaux débouchés en particulier dans la santé, l'énergie, le multimédia, le végétal ou les systèmes urbains. - L'usage de la simulation par calcul intensif constitue une opportunité majeure d'amélioration de la compétitivité des entreprises, en leur permettant de diminuer les coûts et les durées de leurs processus, et d'augmenter la qualité globale de leurs produits et leur capacité d'innovation. De plus, l'usage de ces technologies, qui a longtemps été l'apanage de quelques grands acteurs industriels, devient désormais accessible aux ETI et PME, grâce notamment à des offres en mode «SaaS» (Software as a Service). - La France dispose d'atouts industriels majeurs dans le domaine du calcul intensif et de la simulation numérique. Elle est notamment l'un des rares pays dans le monde à disposer d'acteurs nationaux qui couvrent toute la chaîne de valeur de la simulation numérique. - Le plan a vocation à positionner la France comme un des acteurs mondiaux principaux dans ce domaine. Il a été élaboré à la fois avec les principaux fournisseurs français de technologies et des industriels représentatifs de secteurs utilisateurs. $\bullet$ Les actions proposées visent à la fois à stimuler l'offre technologique française, à mettre en place les outils logiciels dans de nombreuses filières industrielles et à favoriser la diffusion de la simulation auprès des entreprises utilisatrices, notamment dans des secteurs industriels dans lesquels elle n'est actuellement que peu utilisée. Le plan vise notamment une large diffusion de la simulation vers les PME et ETI et comporte un volet essentiel de formation. Sa mise en œuvre s'appuiera sur une déclinaison forte au niveau régional. 


\section{ÉQUIPE DU PLAN}

CHEF DE PROJET

Bruno Bonnell est président du syndicat professionnel Syrobo.

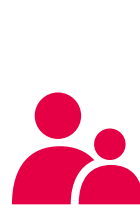

LES INDUSTRIELS

Syrobo et Symop et leurs adhérents Les membres des Groupes de Travail du Comité Robotique "Filière de demain " (France Robots Initiatives)

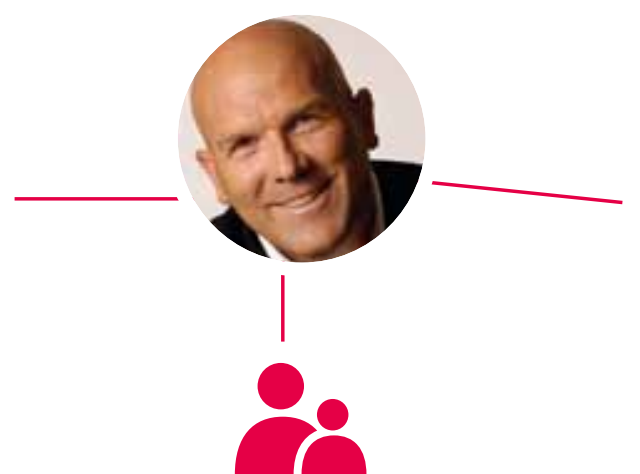

LES ECOLES ET ORGANISMES

DE RECHERCHE

CEA, pôles de compétitivité

Cap Digital et Viaméca, CNRS (GdR)

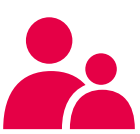

LES ACTEURS PUBLICS MEIN, CGI, MENESR, ANR

\section{SYNTHÈSE DES ACTIONS DU PLAN}

\begin{tabular}{|c|c|c|c|c|}
\hline Action & Pilote & Calendrier & Acteurs publics et privés & $\begin{array}{l}\text { Finalités/livrables ou } \\
\text { leviers actionnés }\end{array}$ \\
\hline \multicolumn{5}{|l|}{ Fédération } \\
\hline \multirow{2}{*}{$\begin{array}{l}\text { Création d'un groupe } \\
\text { de travail Marchés au } \\
\text { sein du comité robotique }\end{array}$} & \multirow[t]{2}{*}{ MEIN } & & \multirow{2}{*}{$\begin{array}{l}\text { Acteurs du secteur, Laboratoires, } \\
\text { MENESR, MEIN, Régions }\end{array}$} & \multirow{2}{*}{$\begin{array}{l}\text { Rapport sur les modalités de } \\
\text { soutien à la création de lieux } \\
\text { de structuration d'écosystèmes }\end{array}$} \\
\hline & & 2015 (rapport) & & \\
\hline \multicolumn{5}{|l|}{ Rayonnement International } \\
\hline \multirow{2}{*}{$\begin{array}{l}\text { Augmentation de la } \\
\text { participation française } \\
\text { dans les programmes } \\
\text { européens }\end{array}$} & \multirow[t]{2}{*}{ MENESR } & \multirow[t]{2}{*}{$2014-2015$} & \multirow[t]{2}{*}{$\begin{array}{l}\text { Entreprises du secteur, Syrobo, } \\
\text { Symop, CNRS, Universités }\end{array}$} & $\begin{array}{l}\text { Création de cellules de soutien } \\
\text { pour le dépôt de projets } \\
\text { européens }\end{array}$ \\
\hline & & & & Lobbying au niveau européen \\
\hline \multicolumn{5}{|c|}{ Popularisation auprès des acteurs économiques } \\
\hline $\begin{array}{l}\text { Organisation d'un } \\
\text { concours }\end{array}$ & MEIN & $2014-2015$ & $\begin{array}{l}\text { Entreprises du secteur, CEA, } \\
\text { Laboratoires, MENESR }\end{array}$ & $\begin{array}{l}\text { Financement par la DGCIS } \\
\text { de } 300 \mathrm{k} €\end{array}$ \\
\hline $\begin{array}{l}\text { Création d'une } \\
\text { plateforme industrielle } \\
\text { robotique }\end{array}$ & CEA & $\begin{array}{l}2015 \text { (réalisation } \\
\text { opérationnelle) } \\
2016 \text { (premier } \\
\text { environnement } \\
\text { fonctionnel) }\end{array}$ & $\begin{array}{l}\text { Industriels fondateurs (ex. Renault, } \\
\text { PSA, Safran, Dassault Aviation, } \\
\text { Dassault Systèmes etc.), industriels } \\
\text { utilisateurs robotique, industriels } \\
\text { fournisseurs technologiques CEA, } \\
\text { CETIM, ENSAM, SYMOP, CNRS/GDR, } \\
\text { CGI }\end{array}$ & $\begin{array}{l}\text { Financement par les } \\
\text { collectivités locales et le PIA }\end{array}$ \\
\hline \multicolumn{5}{|l|}{ Financement } \\
\hline Fonds d'investissement & Robolution & Juillet 2014 & Startups, Bpifrance & $\begin{array}{l}\text { Réalisation des premiers } \\
\text { investissements }\end{array}$ \\
\hline Start PME & MEIN & Action lancée & PME, Symop, CGI, CEA, Cetim & $\begin{array}{l}\text { Soutien financier et technique } \\
\text { aux PME primo-accédantes } \\
\text { à la robotisation (PIA) }\end{array}$ \\
\hline \multicolumn{5}{|c|}{ Recherche et Développement } \\
\hline $\begin{array}{l}\text { Soutien aux projets } \\
\text { R\&D robotiques dans } \\
\text { les guichets existants }\end{array}$ & $\begin{array}{l}\text { MEIN, } \\
\text { ANR, DGA }\end{array}$ & $\begin{array}{l}\text { En cours (selon périodicité } \\
\text { des guichets) }\end{array}$ & $\begin{array}{l}\text { Entreprises robotiques ANR - DGA - } \\
\text { CGI - BPI }\end{array}$ & $\begin{array}{l}\text { Financement par le FUI, le PIA } \\
\text { (PSPC, LEOC), le Rapid }\end{array}$ \\
\hline $\begin{array}{l}\text { Mise en œuvre de } \\
\text { Challenges robotiques }\end{array}$ & ANR & Juin 2014 & Entreprises & Comité de sélection ANR \\
\hline \multicolumn{5}{|l|}{ Formation } \\
\hline \multirow{3}{*}{$\begin{array}{l}\text { Feuille de route } \\
\text { décrivant l'évolution des } \\
\text { formations dans } \\
\text { le domaine }\end{array}$} & \multirow[t]{3}{*}{ MENESR } & $\begin{array}{l}\text { Septembre 2014: groupe } \\
\text { de travail }\end{array}$ & \multirow{3}{*}{$\begin{array}{l}\text { Entreprises du secteur, } \\
\text { établissements d'enseignement } \\
\text { supérieur, organismes de recherche, } \\
\text { MEIN }\end{array}$} & \multirow{3}{*}{$\begin{array}{l}\text { Création d'un GT Formation } \\
\text { au sein du comité de filière } \\
\text { Robotique }\end{array}$} \\
\hline & & $\begin{array}{l}\text { Mars 2015: expression } \\
\text { de besoins }\end{array}$ & & \\
\hline & & Juin 2015: feuille de route & & \\
\hline
\end{tabular}




\section{ROBOTIQUE}
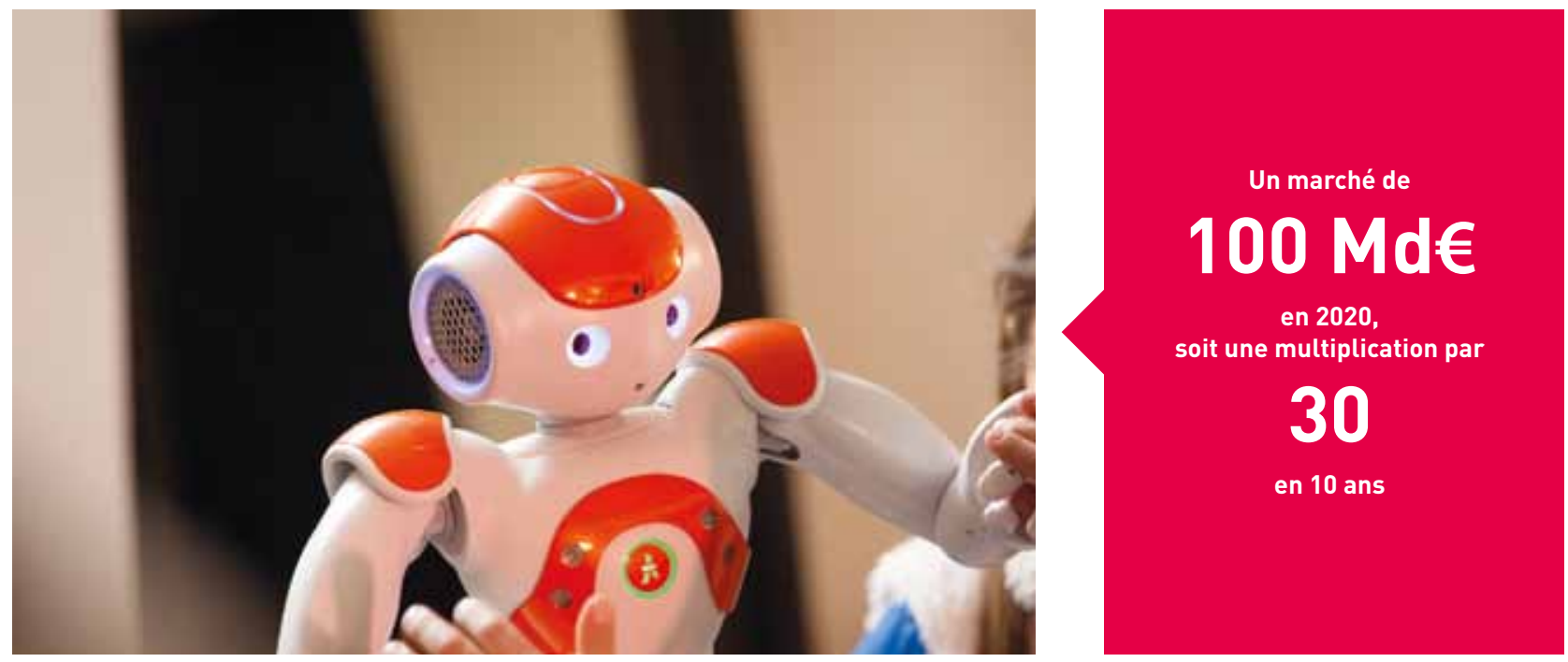

Robot Nao, Aldebaran Robotics

Nous construisons la France de la robotique de pointe. Celle-ci connaîtra dans les prochaines années un essor comparable, selon l'ONU, à celui connu précédemment par l'automobile, l'informatique ou la téléphonie mobile. - Notre pays est déjà l'un des moteurs de la révolution robotique et a le potentiel pour devenir l'un des cinq leaders mondiaux d'ici à 2020. Il dispose d'acteurs industriels et académiques d'excellence, qui sont prêts à relever le défi face aux concurrents américains ou asiatiques. Mais il est cependant peu présent en robotique industrielle et les usines françaises restent insuffisamment robotisées. - La robotique représente une opportunité majeure pour le maintien de la production industrielle et pour les grands défis sociétaux que sont l'assistance aux personnes dépendantes, la santé, l'éducation, l'environnement, la surveillance et la mobilité. - Dans la prolongation de France Robots Initiatives, le plan Robotique entend fédérer et promouvoir la filière, aider au rayonnement international, accélérer la diffusion technologique, soutenir et accompagner des PME sur la voie de la robotisation, permettre le développement de formations pour la robotique contemporaine et lever les freins juridiques et normatifs. - Après le lancement du fonds d'investissement Robolution Capital début 2014, viendra la création d'une plateforme technologique de robotique industrielle en lien avec le plan Usine du futur, le lancement de concours robotiques et l'organisation de challenges pour accélérer les transferts technologiques. 


\section{ÉQUIPE DU PLAN}

CHEF DE PROJET

Guillaume Poupard est directeur général de l'ANSSI

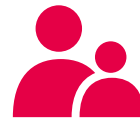

LES INDUSTRIELS

The Greenbow, Prim'x, Bull,

Dictao, Ercom, Gemalto, STMicroelectronics, Thalès,

Communications\&Security, Airbus Defence\&Space, Solucom,

Sogeti, Oppida, Amossys,

Trusted Labs, CIGREF, GITSIS,

CESIN, CLUSIF

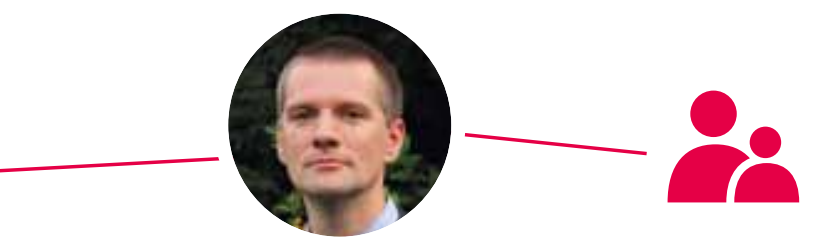

LES ACTEURS PUBLICS

MEIN, DGCIS, INRIA, DGA,

CGI, BPIFrance, SGDSN, ANSSI

\section{SYNTHÈSE DES ACTIONS DU PLAN}

Action

Résultat attendu

(point de vue industriel)

Délai* (court/moyen)

Objectif : Accroitre significativement la demande en solutions de confiance

Sensibiliser à la cybersécurité en renforçant le niveau de compréhension de la menace et développer sa prise en compte au cour de la gouvernance des entreprises et dans l'ensemble des 34 plans industriels

Créer un label France pour les offres nationales, en faire une marque de confiance, de qualité et de performance,

et privilégier le label France dans les achats publics

Croissance des achats des produits

et services des offreurs français

(plus de $20 \%$ par an). Développement Résultats à court terme

du marché très attendu de l'identité

numérique. Ce marché est selon les

études estimé entre $500 \mathrm{M€}$ et $2 \mathrm{Md€}$

\section{Objectif : Développer pour les besoins de la France des offres de confiance}

Mieux piloter et valoriser la R\&D en cybersécurité notamment grâce à l'identification à partir d'une cartographie des acteurs et des segments de marché, des forces, faiblesses, trous capacitaires et orientations en matière de R\&D. Mettre en place une ou des plateformes de cybersécurité, de tests et de démonstrations
Développement de 2 à 3 nouvelles gammes par an d'offres répondant à un besoin avéré et meilleure utilisation des dispositifs d'investissement

\section{Objectif : Organiser la conquête des marchés à l'étranger}

Renforcer la connaissance de l'offre française à

l'international, déployer un forum cybersécurité public-privé

et soutenir les exportations du secteur cybersécurité.

Renforcer l'influence de la France en engageant et renforçant $\quad \begin{aligned} & \text { Accroissement des parts de marchés } \\ & \text { des industriels français à l'export }\end{aligned}$

les actions de normalisation dans les domaines de la

Court à moyen terme

cybersécurité non encore matures pour lesquels l'industrie

nationale peut se positionner en tant que leader européen

Organiser un concours d'idées pour l'émergence et le

déploiement de solutions innovantes portées par des start-up

Gagner de la souplesse à l'export Court terme

Objectif : Renforcer les entreprises nationales du domaine de la cybersécurité

Renforcer la formation et l'entraînement des spécialistes

Faire croître les ressources compétentes (plus de $10 \%$ par an)

et experts du domaine

$\begin{array}{ll}\begin{array}{l}\text { Création d'un fonds d'investissement privé permettant } \\ \text { de consolider la filière }\end{array} & \begin{array}{l}\text { Meilleure utilisation des dispositifs } \\ \text { et valorisation des travaux. Création }\end{array}\end{array}$

de consolider la filière

de champions français à l'échelle

Court terme (appui sur le travail existant: ACN et ANR/FUI/FSN)

européenne ( 1 sur 3 ans) 


\section{CYBERSÉCURITÉ}
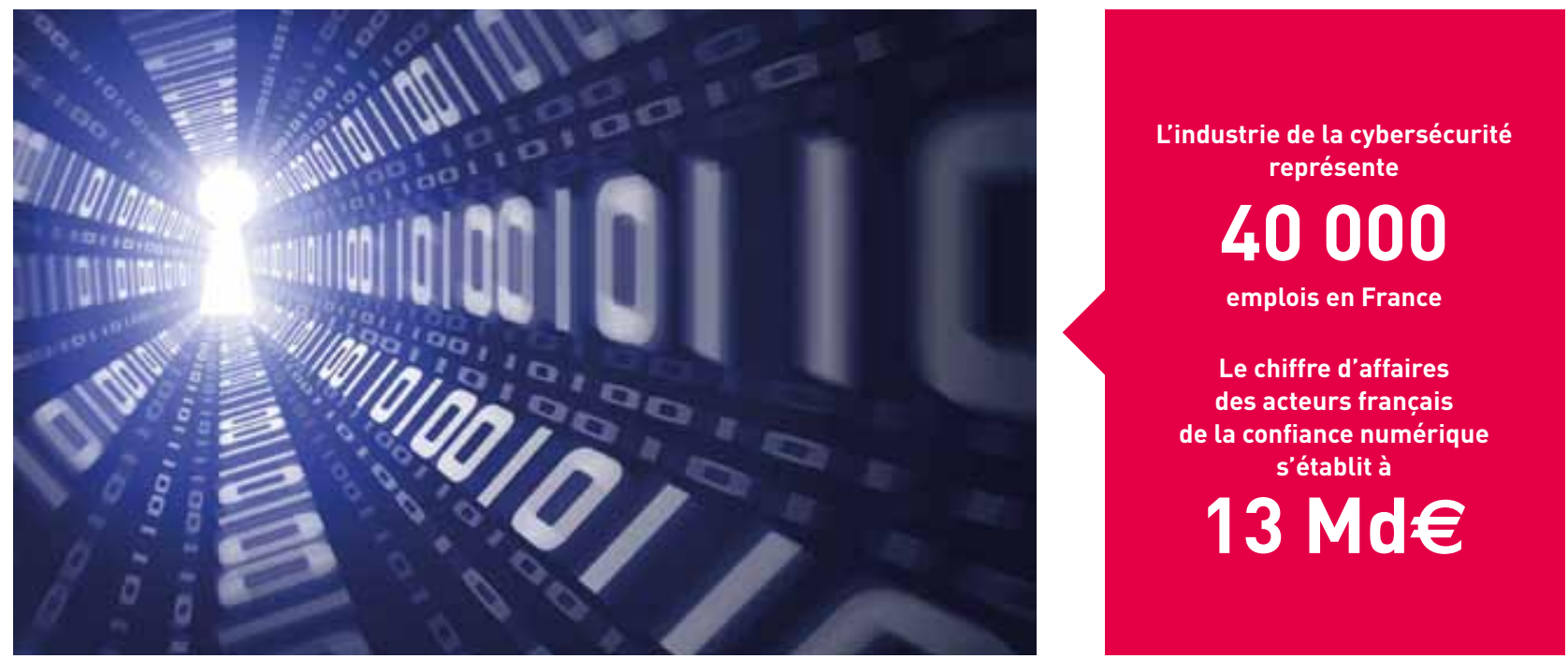

Échange sécurisé d'informations

Nous construisons la France de la sécurité et de la confiance numérique. Dans la construction de la France numérique, le développement d'une industrie performante en matière de cybersécurité est une nécessité, un enjeu de souveraineté et une opportunité. Une nécessité, car nous devons sécuriser nos infrastructures les plus vitales pour assurer la défense comme la sécurité de notre pays et protéger la vie quotidienne des Français. Un enjeu de souveraineté, car pour protéger nos secrets numériques les plus essentiels, nous ne pouvons dépendre que de nous. Une opportunité, car elle est une formidable source de créations d'emplois. La France possède les acteurs industriels de rang mondial et le tissu de PME capables de relever ce défi. - Le plan poursuit quatre objectifs: 1. Accroître significativement la demande en solutions de cybersécurité de confiance ; 2 . Développer pour les besoins de la France des offres de confiance; 3. Organiser la conquête des marchés à l'étranger ; 4. Renforcer les entreprises nationales du domaine cybersécurité. - Si une offre de confiance nationale existe et est disponible, elle est néanmoins souvent méconnue des commanditaires publics et privés nationaux, voire ignorée face aux offres étrangères au marketing bien plus agressif, alors qu'elle couvre un besoin de sécurité qui va croissant. Il en va de même à l'export, où nous ne valorisons pas suffisamment la qualité des offres. Cette offre de confiance mérite d'être enrichie, pour répondre aux ruptures technologiques : un effort de gouvernance et de valorisation de la Recherche et Développement sera fait pour tirer tout le bénéfice d'un tissu industriel national large, mais parfois trop dispersé (plus de 600 acteurs). La consolidation de ce tissu industriel doit d'ailleurs être un objectif de l'État, notamment au regard des risques de concurrence stérile ou de rachats inappropriés portés par l'éparpillement actuel, mais surtout devant les formidables opportunités de parts de marché que les regroupements ou adossements peuvent offrir. 


\title{
ÉQUIPE DU PLAN
}

\author{
CHEFS DE PROJET
}

Frédéric Sanchez est président du Directoire du groupe Fives. Bernard Charlès est PDG de Dassault Systèmes.

Airbus, Coriolis Composites, EDF, Gorgé, PSA, Renault, Schneider, Staubli, Air Liquide, Alstom, CORAC, Daher, DCNS, Essilor, GSK, Michelin, Lafarge, SEB, Safran, Sogeti, Solvay, TriballatNoyal, Trimet, Valeo
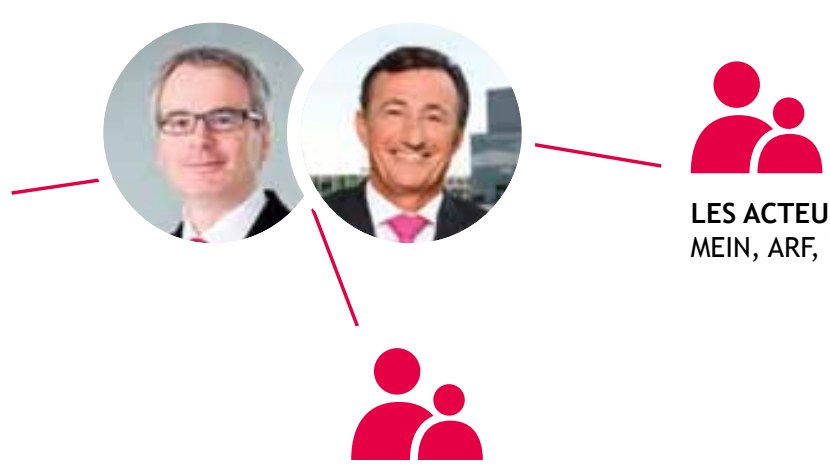

LES ACTEURS PUBLICS

MEIN, ARF, Bpifrance, CGI

LES ÉCOLES ET ORGANISMES DE RECHERCHE

CEA, CGI, IRT Jules Verne

\section{SYNTHÈSE DES ACTIONS DU PLAN}
Action
Pilote
Calendrier
Acteurs publics
Finalités/livrables

et leviers d'action

Accompagnement des PME et des ETI dans leur diagnostic et leurs actions de modernisation

\begin{tabular}{|c|c|c|c|c|}
\hline Plan de financement des équipements & MEIN & 2014-2017 & MEIN, CGI, Bpifrance & $\begin{array}{l}\text { Mise à niveau des systèmes } \\
\text { de production français, tout } \\
\text { en les dotant des derniers } \\
\text { équipements, } \\
\text { à la pointe de la compétitivité }\end{array}$ \\
\hline $\begin{array}{l}\text { Programme } 5 \mathrm{E}: \text { : Efficacité Énergétique } \\
\text { et Empreinte Environnementale des } \\
\text { Entreprises» }\end{array}$ & CDC Climat & $\begin{array}{l}2014 \text { pour le montage } \\
\text { du financement } \\
2015-2017 \text { pour } \\
\text { les projets }\end{array}$ & MEIN & $\begin{array}{l}\text { Projets de financement } \\
\text { des éco-équipements pour } \\
\text { l'Efficacité Énergétique et } \\
\text { l'Empreinte Environnementale } \\
\text { des Entreprises }\end{array}$ \\
\hline $\begin{array}{l}\text { Programmes régionaux d'excellence } \\
\text { industrielle des PMEs et ETIs }\end{array}$ & ARF, Régions & $2014-2015$ & $\begin{array}{l}\text { Financement national } \\
\text { pour la méthode } \\
\text { et la synthèse et régional } \\
\text { pour les actions }\end{array}$ & $\begin{array}{l}\text { Développement d'un solide } \\
\text { tissu de PME compétitives, } \\
\text { bien insérées dans les filières } \\
\text { globales et capables de } \\
\text { contribuer aux procédés les } \\
\text { plus en pointe }\end{array}$ \\
\hline
\end{tabular}

Mise en place de lignes de production pilotes, vitrines du savoir-faire francais

Première vague Triballat Noyal, Areva Première vague:

Les projets pré-sélectionnés: programme Wind, Daher, PSA, Air 7 projets lancés

pilote 5 e dans l'agroalimentaire,

eoliennes off shore au Havre,

ligne flexible et automatisée pour

petites pièces en composites dans

l'aéronautique, ligne Composites

de bouteilles de gaz liquide, MIM chez

Safran, chantiers navals en Bretagne.

Liquide, Safran, DCNS 4 e trimestre 2014

Deuxième vague

11 projets pilotes
Trimet, Solvay, PFA, de projets labellisés

Trimet, Solvay, PFA, à planifier

SEB, Valeo Eclairage

Technip, Emin

Leydier, CEA/

Diotasoft, Enersens
Preuve des concepts

MEIN, Régions,

Pôles de compétitivité, technologies

Bpifrance... $\quad$ Vitrine pour «l'Usine

Française »

\section{Projets de R\&D}

Impression 3D

Contrôle non-destructif

Plateforme Robotique Industrielle

Virtualisation et Internet des objets

Composites

Place de l'homme dans l'Usine

\begin{tabular}{|c|c|c|c|}
\hline $\begin{array}{l}\text { Gorgé } \\
\text { CEA } \\
\text { CEA et Staubli } \\
\text { 3DS } \\
\text { Fives } \\
\text { Staubli, 3DS, } \\
\text { Fives CFA }\end{array}$ & $\begin{array}{l}\text { Montage: } 2014 \text {, } \\
\text { Recherches: } \\
\text { 2015-2017 }\end{array}$ & $\begin{array}{l}\text { MEIN, CGI, Régions, Pôles } \\
\text { de compétitivité }\end{array}$ & $\begin{array}{l}\text { Développement d'un } \\
\text { leadership technologique } \\
\text { sur les thématiques clés non } \\
\text { matures de l'usine du futur }\end{array}$ \\
\hline
\end{tabular}




\section{USINE DU FUTUR}
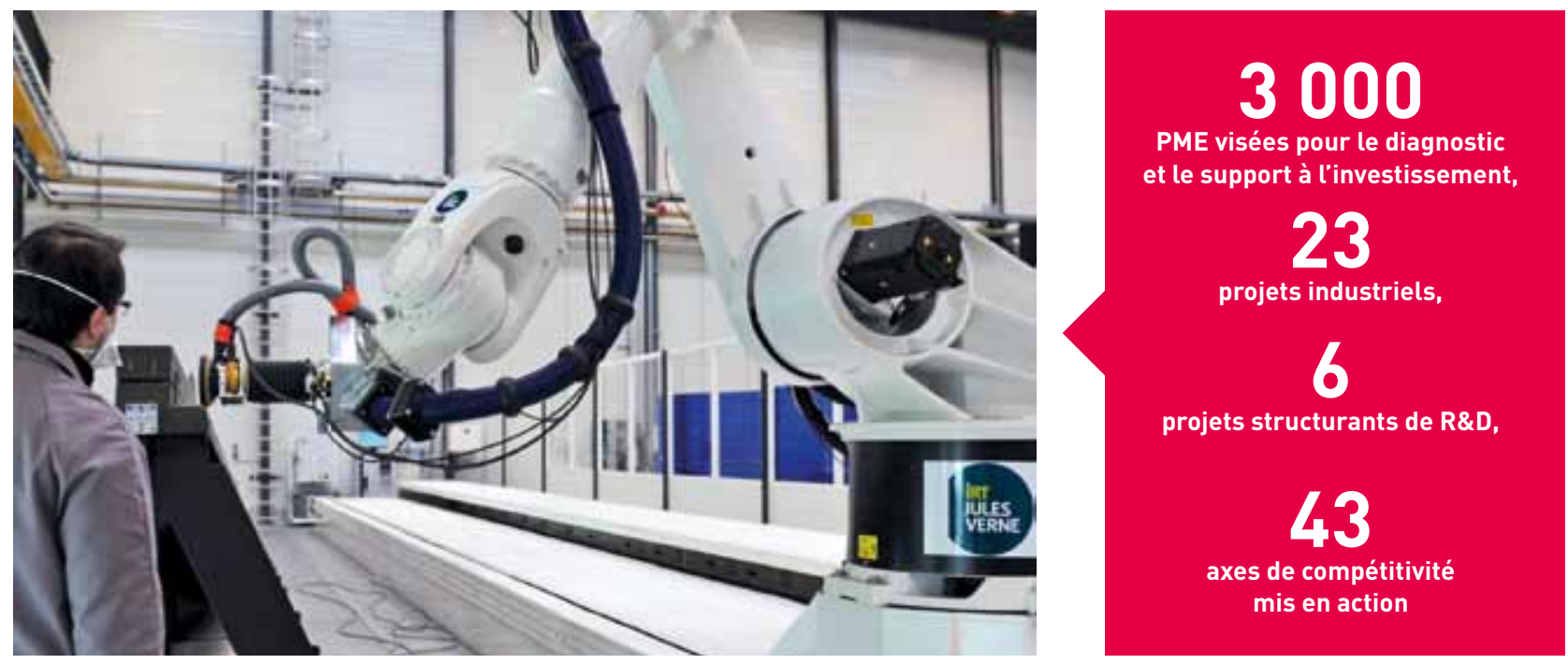

Bras robotisé, Institut de Recherche Technologique Jules Verne

- Nous construisons la France de la troisième révolution industrielle. Pour retrouver son rang dans la bataille mondiale, gagner en compétitivité, maintenir de l'emploi industriel et améliorer la place de l'homme dans l'usine, la France doit relever le double défi de moderniser son outil productif et de concevoir et de développer les processus et les outils de production de l'avenir. L'usine de demain devra être plus écologique et sobre en ressources, plus intelligente, avec des modes de production toujours plus adaptés à des productions personnalisées ou en petite série. Elle devra remettre l'humain au cœur de la relation homme-machine et être plus proche de son écosystème local (clients, sous-traitants et fournisseurs). Le plan «Usine du futur » permettra à la France d'être au rendez-vous de la troisième Révolution industrielle, au carrefour des transitions énergétique et numérique de notre société. - Pour mettre en place cette usine d'excellence, le plan a identifié les facteurs clés de succès pour nos filières industrielles dans l'avenir et veut faire émerger une offre française de technologies et d'accompagnement du changement. Il se donne pour objectif de coordonner les briques technologiques existantes et de développer celles qui manquent encore à notre écosystème grâce à des projets de R\&D financés par l'État. Il prévoit d'autre part, la mise en place de lignes de production pilotes dans des entreprises emblématiques qui seront les vitrines du savoir-faire français. $\bullet$ Pour le déploiement national des technologies et méthodes d'excellence de ces lignes pilotes, le cœur du plan consiste en un programme d'accompagnement méthodologique et financier des PME et des ETI sous le co-pilotage des Régions qui financeront des diagnostics industriels pour 2000 à 3000 d'entre elles. Le financement des projets de modernisation de ces entreprises et des autres pourra se faire dans des délais très courts par une gamme de prêts de Bpifrance labellisés «Usine du futur» cumulables jusqu'à 12 M€ par entreprise et par le biais d'un amortissement accéléré. 


\section{LES CHEFS DE PROJET}

- ÉNERGIES RENOUVELABLES Jean-Claude Andréini - LA VOITURE POUR TOUS CONSOMMANT MOINS DE 2 LITRES AUX 100 KM Jean-Michel Billig, Gilles Le Borgne - BORNES ÉLECTRIQUES DE RECHARGE Francis Vuibert - AUTONOMIE ET PUISSANCE DES BATTERIES Florence Lambert • VÉHICULE AUTONOME Carlos Ghosn - AVION ÉLECTRIQUE ET NOUVELLE GÉNÉRATION D’AÉRONEFS Jean J. Botti — DIRIGEABLES ET DRONES CIVILS Jean-Yves Longère, Bruno Even, Francis Durufle - LOGICIELS ET SYSTĖMES EMBARQUÉS Éric Bantegnie - SATELLITES À PROPULSION ÉLECTRIQUE Jean-Yves Le Gall • TGV DU FUTUR Henri Poupart-Lafarge - NAVIRES ÉCOLOGIQUES Jean-Marie Poimbœuf - TEXTILES TECHNIQUES ET INTELLIGENTS Yves Dubief - INDUSTRIES DU BOIS Franck Mathis, Dominique Weber — RECYCLAGE ET MATÉRIAUX VERTS Antoine Frérot RÉNOVATION THERMIQUE DES BÂTIMENTS Jacques Pestre, Marcel Torrents - RÉSEAUX ÉleCTRIQUES INTELLIGENTS Dominique Maillard - QUALITÉ DE L'EAU ET GESTION DE LA RARETÉ Jean-Louis Chaussade, Christophe Chevillion - CHIMIE VERTE ET BIOCARBURANTS Pascal Barthélemy - BIOTECHNOLOGIES MÉDICALES André Choulika - SANTÉ NUMÉRIQUE Mireille Faugère, Christian Nibourel - dispositiFs médicauX et nOUVEaUX ÉQUIPEMENTS DE SANTÉ André-Michel Ballester, Sacha Loiseau - PRODUITS INNOVANTS POUR UNE ALIMENTATION SÛRE, SAINE ET DURABLE Jean-Philippe Girard — BIG DATA Paul Hermelin, François Bourdoncle - CLOUD COMPUTING Thierry Breton, Octave Klaba - E-ÉDUCATION Jean-Yves Hepp, Deborah Elalouf - SOUVERAINETÉ TÉLÉCOMS Philippe Keryer • NANOÉLECTRONIQUE Laurent Malier - OBJETS CONNECTÉS Eric Carreel — RÉALITÉ AUGMENTÉE Vincent Marcatté — SERVICES SANS CONTACT Olivier Piou - SUPERCALCULATEURS Gérard Roucairol • ROBOTIQUE Bruno Bonnell - CYBERSÉCURITÉ Guillaume Poupard — USINE DU FUTUR Frédéric Sanchez, Bernard Charlès 


\section{GLOSSAIRE}

\section{LES ACTEURS PUBLICS}

ADEME : Agence de l'Environnement et de la Maîtrise de l'Énergie AFIMB : Agence française pour l'information multimodale et la billettique ANSSI: Agence Nationale de la Sécurité des Systèmes d'Information ANSEM: Agence Nationale de SEcurité du Médicament et des produits de santé AP-HP: Assistance Publique - Hôpitaux de Paris ARF : Association des Régions de France Bpifrance: Banque Publique d'Investissement CDC: Caisse des Dépôts et Consignations CEPS: Comité Économique des Produits de Santé $\mathrm{CGI}$ : Commissariat Général à l'Investissement CMN: Centre des Monuments Nationaux COSEI : COmité Stratégique de filière Éco-Industries

CRE: Commission de Régulation de l'Énergie Direccte: Directions régionales des entreprises, de la concurrence, de la consommation, du travail et de l'emploi DGA: Direction Générale de l'Armement DGAC: Direction Générale de l'Aviation Civile DGCIS: Direction Générale de la Compétitivité, de l'Industrie et des Services DGPAAT: Direction Générale des Politiques Agricole, Agroalimentaire et des Territoires DHUP: Direction de l'Habitat, de l'Urbanisme et des Paysages

EMA: European Medicines Agency

ERDF : Électricité Réseau Distribution France

EPSF : Établissement Public de Sécurité Ferroviaire

FEADER: Fonds Européen Agricole pour le DEveloppement Rural FEDER: Fonds Européen de DEveloppement Régional

HAS: Haute Autorité de Santé IGN: Institut Géographique National MAAF : Ministère de l'Agriculture, de l'Agroalimentaire et de la Forêt MDREFP: Ministre de la Décentralisation, de la Réforme de l'État et de la Fonction Publique MEDDE: Ministère de l'Écologie, du Développement Durable et de l'Énergie MEIN : Ministère de l'Économie, de l'Industrie et du Numérique MENESR: Ministère de l'Éducation, de l'Enseignement Supérieur et de la Recherche MLET: Ministère du Logement et de l'Égalité des Territoires MINDEF: Ministère de la Défense Rmn-GP: Réunion des musées nationaux - Grand Palais RTE: Réseau de Transport d'Électricité SETMP: Secrétariat d'État aux Transports, à la Mer et à la Pêche SGDSN: Secrétariat Général de la Défense et de la Sécurité Nationale SGMAP: Secrétariat Général pour la Modernisation de l'Action Publique

\section{LES ÉCOLES ET ORGANISMES DE RECHERCHE}

AVIESAN: Alliance pour les sciences de la vie et de la santé ANR: Agence Nationale de la Recherche CEA: Commissariat à l'Énergie Atomique et aux énergies alternatives CEA-LETI: Laboratoire d'Électronique et de Technologie de l'Information du CEA.

CNRS: Centre National de la Recherche Scientifique

ENAC: École Nationale de l'Aviation Civile

ENSAE: École Nationale de la Statistique et de l'Administration Économique

ENSAI: École Nationale de la Statistique et de l'Analyse de l'Information FEM-TO: Franche-Comté Électronique, Mécanique, Thermique et Optique GdR: Groupement de Recherche (CNRS)

IFSTTAR: Institut français des sciences et technologies des transports, de l'aménagement et des réseaux 
INPG : Institut National Polytechnique de Grenoble

INRIA: Institut National de Recherche en Informatique et en Automatique INSERM: Institut National de la Santé Et de la Recherche Médicale

IMT: Institut Mines-Télécom

IRSTEA: Institut de Recherche en Sciences et Technologies pour l'Environnement et l'Agriculture

IRT: Institut de Recherche Technologique

ITE: Institut pour la Transition Énergétique

ITMO: Institut Thématique Multi-Organismes

ONERA: Office National d'Études et de Recherches Aérospatiales

RS2E : Réseau de recherche et technologie sur le stockage électrochimique de l'énergie

\section{LES ASSOCIATIONS OU FÉDÉRATIONS}

AFRV : Association française de Réalité Virtuelle, Augmentée, Mixte et d'Interaction 3D

AVERE: Association pour le développement de la mobilité électrique

CAPEB : Confédération de l'Artisanat et des Petites Entreprises du Bâtiment

CEREMH: Centre de Ressources et d'innovation Mobilité Handicap

CETIM: Centre technique des industries mécaniques

CORAC: COnseil pour la Recherche Aéronautique Civile

FEDEREC : Fédération des Entreprises de Recyclage

FFB : Fédération Française du Bâtiment

FNADE: Fédération Nationale des Activités de la Dépollution et de l'Environnement

GICAN: Groupement des Industries de Construction et Activités Navales

GIFAS: Groupement des Industries Françaises Aéronautiques et Spatiales

IFPen: Institut Français du Pétrole Énergies Nouvelles

IFTH: Institut Français du Textile et de l'Habillement

LEEM: Les entreprises du médicament

NOVEA: Association pour la diffusion des nouvelles technologies au service du développement durable

PFA: Plateforme de la Filière Automobile

SYMOP: syndicat des Machines et technologies de Production

SYROBO: Syndicat de la robotique de service professionnel et personnel

UIC : Union des industries chimiques

\section{LES DISPOSITIFS PUBLICS}

JESSICA: Joint European Support for Sustainable Investment in City Areas (alliance européenne d'appui aux investissements durables en zone urbaine)

FUI: Fonds unique interministériel

PIA: Programme des Investissements d'Avenir

PIPC: Prêt à l'industrialisation des projets de recherche et développement issus des pôles

de compétitivité (Bpifrance)

RGE : Reconnu Garant de l'Environnement

LPTE: Loi Pour la Transition Énergétique

\section{LA TERMINOLOGIE PROFESSIONNELLE}

ENR: Énergies Renouvelables

IHM: Interface Homme Machine

ITS: Intelligent Transport Systems

GNL: Gaz Naturel Liquéfié

TGAP: Taxe Générale sur les Activités Polluantes

ITAR: International Traffic in Arm Regulations (réglementation américaine sur le trafic d'armes au niveau international)

TPE: Terminal de Paiement Électronique

JESSICA: Joint European Support for Sustainable Investment in City Areas (alliance européenne d'appui aux investissements durables en zone urbaine) 


\section{WWW.GOUVERNEMENT.FR WWW.ECONOMIE.GOUV.FR}

